\title{
A unified approach to the theory of separately holomorphic mappings
}

\author{
VIÊT-ANH NGUYÊN
}

\begin{abstract}
We extend the theory of separately holomorphic mappings between complex analytic spaces. Our method is based on Poletsky theory of discs, Rosay theorem on holomorphic discs and our recent joint-work with Pflug on boundary cross theorems in dimension 1. It also relies on our new technique of conformal mappings and a generalization of Siciak's relative extremal function. Our approach illustrates the unified character: "From local information to global extensions". Moreover, it avoids systematically the use of the classical method of doubly orthogonal bases of Bergman type.
\end{abstract}

Mathematics Subject Classification (2000): 32D15 (primary); 32D10 (secondary).

\section{Introduction}

In this article all complex manifolds are supposed to be of finite dimension and countable at infinity, and all complex analytic spaces are supposed to be reduced, irreducible, of finite dimension and countable at infinity. For a subset $S$ of a topological space $M, \bar{S}$ denotes the closure of $S$ in $M$, and the set $\partial S:=\bar{S} \cap \overline{M \backslash S}$ denotes, as usual, the boundary of $S$ in $M$.

The main purpose of this work is to investigate the following:

Problem 1.1. Let $X, Y$ be two complex manifolds, let $D$ (respectively $G$ ) be an open subset of $X$ (respectively $Y$ ), let $A$ (respectively $B$ ) be a subset of $\bar{D}$ (respectively $\bar{G}$ ) and let $Z$ be a complex analytic space. Define the cross

$$
W:=((D \cup A) \times B) \bigcup(A \times(G \cup B)) .
$$

We want to determine the "envelope of holomorphy" of the cross $W$, that is, an "optimal" open subset of $X \times Y$, denoted by $\widehat{\widetilde{W}}$, which is characterized by the following properties:

Let $f: W \longrightarrow Z$ be a mapping that satisfies, in essence, the following two conditions:

Received May 15, 2007; accepted in revised form January 8, 2008. 
- $f(a, \cdot)$ is holomorphic on $G$ for all $a \in A, f(\cdot, b)$ is holomorphic on $D$ for all $b \in B$

- $f(a, \cdot)$ is continuous on $G \cup B$ for all $a \in A, f(\cdot, b)$ is continuous on $D \cup A$ for all $b \in B$.

Then there is a holomorphic mapping $\hat{f}$ defined on $\widehat{\widetilde{W}}$ such that for every $(\zeta, \eta) \in$ $W, \hat{f}(z, w)$ tends to $f(\zeta, \eta)$ as $(z, w) \in \widehat{\widetilde{W}}$ tends, in some sense, to $(\zeta, \eta)$.

Now we recall briefly the main developments around this problem. All the results obtained so far may be divided into two directions. The first direction investigates the results in the "interior" context: $A \subset D$ and $B \subset G$, while the second one explores the "boundary" context: $A \subset \partial D$ and $B \subset \partial G$.

The first fundamental result in the field of separate holomorphy is the wellknown Hartogs extension theorem for separately holomorphic functions (see [14]). In the language of the Problem 1.1 the following case $X=\mathbb{C}^{n}, Y=\mathbb{C}^{m}, A=$ $D, B=G, Z=\mathbb{C}$ has been solved and the result is $\widehat{\widetilde{W}}=D \times G$. In particular, this theorem may be considered as the first main result in the first direction. In his famous article [8] Bernstein obtained some positive results for the Problem 1.1 in certain cases where $A \subset D, B \subset G, X=Y=\mathbb{C}$ and $Z=\mathbb{C}$.

More than 60 years later, a next important impetus was made by Siciak (see [44, 45]) in 1969-1970, where he established some significant generalizations of the Hartogs extension theorem. In fact, Siciak's formulation of these generalizations gives rise to the above Problem 1.1: to determine the envelope of holomorphy for separately holomorphic functions defined on some cross sets $W$. The theorems obtained under this formulation are often called cross theorems. Using the so-called relative extremal function, Siciak completed the Problem 1.1 for the case where $A \subset D, B \subset G, X=Y=\mathbb{C}$ and $Z=\mathbb{C}$.

The next deep steps were initiated by Zahariuta in 1976 (see [46]) when he started to use the method of common bases of Hilbert spaces. This original approach permitted him to obtain new cross theorems for some cases where $A \subset$ $D, B \subset G$ and $D=X, G=Y$ are Stein manifolds. As a consequence, he was able to generalize the result of Siciak in higher dimensions.

Later, Nguyên Thanh Vân and Zeriahi (see [25-27]) developed the method of doubly orthogonal bases of Bergman type in order to generalize the result of $\mathrm{Za}$ hariuta. This is a significantly simpler and more constructive version of Zahariuta's original method. Nguyên Thanh Vân and Zeriahi have recently achieved an elegant improvement of their method (see [24,48]).

Using Siciak's method, Shiffman (see [42]) was the first to generalize some Siciak's results to separately holomorphic mappings with values in a complex analytic space Z. Shiffman's result (see [43]) shows that the natural "target spaces" for obtaining satisfactory generalizations of cross theorems are the ones which possess the Hartogs extension property (see Subsection 2.4 below for more explanations).

In 2001 Alehyane and Zeriahi solved the Problem 1.1 for the case where $A \subset$ $D, B \subset G$ and $X, Y$ are Stein manifolds, and $Z$ is a complex analytic space which possesses the Hartogs extension property (see [5, Theorem 2.2.4]). 
In a recent work (see [28]) we complete, in some sense, the Problem 1.1 for the case where $A \subset D, B \subset G$ and $X, Y$ are arbitrary complex manifolds. The main ingredients in our approach are Poletsky theory of discs developed in $[38,39]$, Rosay's theorem on holomorphic discs (see [41]), the above mentioned result of Alehyane-Zeriahi and the technique of level sets of the plurisubharmonic measure which was previously introduced in our joint-work with Pflug (see [34]).

To conclude the first direction of research we mention the survey articles by Nguyên Thanh Vân [23] and Peter Pflug [33] which give nice accounts on this subject.

The first result in the second direction (i.e. "boundary context") was established in the work of Malgrange-Zerner [47] in the 1960s. Further results in this direction were obtained by Komatsu [21] and Drużkowski [9], but only for some special cases. Recently, Gonchar $[12,13]$ has proved a more general result where the following case has been solved: $X=Y=\mathbb{C}, D$ and $G$ are Jordan domains, $A$ (respectively $B$ ) is an open boundary subset of $\partial D$ (respectively $\partial G$ ), and $Z=\mathbb{C}$. It should be noted that Airapetyan and Henkin published a general version of the edge-of-the-wedge theorem for CR manifolds (see [1] for a brief version and [2] for a complete proof). Gonchar's result could be deduced from the latter works. In our joint-articles with Pflug (see [34-36]), Gonchar's result has been generalized considerably. More precisely, the work in [36] treats the case where the "source spaces" $X, Y$ are arbitrary complex manifolds, $A$ (respectively $B$ ) is an open boundary subset of $\partial D$ (respectively $\partial G$ ), and $Z=\mathbb{C}$. The work in [35] solves the case where the "source spaces" $X, Y$ are Riemann surfaces, $A$ (respectively $B$ ) is a measurable (boundary) subset of $\partial D$ (respectively $\partial G$ ), and $Z=\mathbb{C}$.

The main purpose of this article is to give a new version of the Hartogs extension theorem which unifies all results up to now. Namely, we are able to give a reasonable solution to the Problem 1.1 when the "target space" $Z$ possesses the Hartogs extension property. Our method is based on a systematic application of Poletsky theory of discs, Rosay theorem on holomorphic discs and our joint-work with Pflug on boundary cross theorems in dimension 1 (see [35]). It also relies on our new technique of conformal mappings and a generalization of Siciak's relative extremal function. The approach illustrates the unified character in the theory of extension of holomorphic mappings:

\section{- One can deduce the global extension from local information.}

Moreover, the novelty of this new approach is that one does not use the classical method of doubly orthogonal bases of Bergman type.

We close the introduction with a brief outline of the paper to follow.

In Section 2 we formulate the main results.

The tools which are needed for the proof of the main results are developed in Section 3, 4, 5 and 7.

The proof of the main results is divided into three parts, which correspond to Section 6, 8 and 9. Section 10 concludes the article with various applications of our results. 
ACKNOWLEDGEMENTS. The paper was written while the author was visiting the Abdus Salam International Centre for Theoretical Physics in Trieste. He wishes to express his gratitude to this organization.

\section{Preliminaries and statement of the main result}

First we develop some new notions such as system of approach regions for an open set in a complex manifold, and the corresponding plurisubharmonic measure. These will provide the framework for an exact formulation of the Problem 1.1 and for our solution.

\subsection{Approach regions, local pluripolarity and plurisubharmonic measure}

Definition 2.1. Let $X$ be a complex manifold and let $D \subset X$ be an open subset. A system of approach regions for $D$ is a collection $\mathcal{A}=\left(\mathcal{A}_{\alpha}(\zeta)\right)_{\zeta \in \bar{D}, \alpha \in I_{\zeta}}$ of open subsets of $D$ with the following properties:

(i) For all $\zeta \in D$, the system $\left(\mathcal{A}_{\alpha}(\zeta)\right)_{\alpha \in I_{\zeta}}$ forms a basis of open neighborhoods of $\zeta$ (i.e., for any open neighborhood $U$ of a point $\zeta \in D$, there is $\alpha \in I_{\zeta}$ such that $\left.\zeta \in \mathcal{A}_{\alpha}(\zeta) \subset U\right)$.

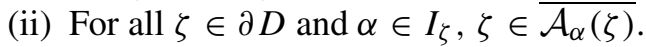

$\mathcal{A}_{\alpha}(\zeta)$ is often called an approach region at $\zeta$.

$\mathcal{A}$ is said to be canonical if it satisfies (i) and the following property (which is stronger than (ii)):

(ii') For every point $\zeta \in \partial D$, there is a basis of open neighborhoods $\left(U_{\alpha}\right)_{\alpha \in I_{\zeta}}$ of $\zeta$ in $X$ such that $\mathcal{A}_{\alpha}(\zeta)=U_{\alpha} \cap D, \alpha \in I_{\zeta}$.

It is possible that $I_{\zeta}=\varnothing$ for some $\zeta \in \partial D$.

Various systems of approach regions which one often encounters in Complex Analysis will be described in the next subsection. Systems of approach regions for $D$ are used to deal with the limit at points in $\bar{D}$ of mappings defined on some open subsets of $D$. Consequently, we deduce from Definition 2.1 that the subfamily $\left(\mathcal{A}_{\alpha}(\zeta)\right)_{\zeta \in D, \alpha \in I_{\zeta}}$ is, in a certain sense, independent of the choice of a system of approach regions $\mathcal{A}$. In addition, any two canonical systems of approach regions are, in some sense, equivalent. These observations lead us to use, throughout the paper, the following convention:

We fix, for every open set $D \subset X$, a canonical system of approach regions. When we want to define a system of approach regions $\mathcal{A}$ for an open set $D \subset X$, we only need to specify the subfamily $\left(\mathcal{A}_{\alpha}(\zeta)\right)_{\zeta \in \partial D, \alpha \in I_{\zeta}}$.

In what follows we fix an open subset $D \subset X$ and a system of approach regions $\mathcal{A}=\left(\mathcal{A}_{\alpha}(\zeta)\right)_{\zeta \in \bar{D}, \alpha \in I_{\zeta}}$ for $D$. 
For every function $u: D \longrightarrow[-\infty, \infty)$, let

$$
(\mathcal{A}-\lim \sup u)(z):= \begin{cases}\sup _{\alpha \in I_{z}} \limsup _{w \in \mathcal{A}_{\alpha}(z), w \rightarrow z} u(w), & z \in \bar{D}, I_{z} \neq \varnothing, \\ \limsup _{w \in D, w \rightarrow z} u(w), & z \in \partial D, I_{z}=\varnothing .\end{cases}
$$

By Definition 2.1 (i), $\left.(\mathcal{A}-\lim \sup u)\right|_{D}$ coincides with the usual upper semicontinuous regularization of $u$.

For a set $A \subset \bar{D}$ put

$$
h_{A, D}:=\sup \{u: u \in \mathcal{P} \mathcal{S H}(D), u \leq 1 \text { on } D, \mathcal{A}-\lim \sup u \leq 0 \text { on } A\},
$$

where $\mathcal{P S H}(D)$ denotes the cone of all functions plurisubharmonic on $D$.

$A$ is said to be pluripolar in $D$ if there is $u \in \mathcal{P S H}(D)$ such that $u$ is not identically $-\infty$ on every connected component of $D$ and $A \subset\{z \in D: u(z)=-\infty\}$. $A$ is said to be locally pluripolar in $D$ if for any $z \in A$, there is an open neighborhood $V \subset D$ of $z$ such that $A \cap V$ is pluripolar in $V$. $A$ is said to be nonpluripolar (respectively non locally pluripolar) if it is not pluripolar (respectively not locally pluripolar). According to a classical result of Josefson and Bedford (see $[6,16])$, if $D$ is a Riemann domain over a Stein manifold, then $A \subset D$ is locally pluripolar if and only if it is pluripolar.

Definition 2.2. The relative extremal function of $A$ relative to $D$ is the function $\omega(\cdot, A, D)$ defined by

$$
\omega(z, A, D)=\omega_{\mathcal{A}}(z, A, D):=\left(\mathcal{A}-\lim \sup h_{A, D}\right)(z), \quad z \in \bar{D} .{ }^{1}
$$

Note that when $A \subset D$, Definition 2.2 coincides with the classical definition of Siciak's relative extremal function.

Next, we say that a set $A \subset \bar{D}$ is locally pluriregular at a point $a \in \bar{A}$ if $\omega(a, A \cap U, D \cap U)=0$ for all open neighborhoods $U$ of $a$. Moreover, $A$ is said to be locally pluriregular if it is locally pluriregular at all points $a \in A$. It should be noted from Definition 2.1 that if $a \in \bar{A} \cap D$ then the property of local pluriregularity of $A$ at $a$ does not depend on any particular choices of a system of approach regions $\mathcal{A}$, while the situation is different when $a \in \bar{A} \cap \partial D$ : the property does depend on $\mathcal{A}$.

We denote by $A^{*}$ the following set

$$
(A \cap \partial D) \bigcup\{a \in \bar{A} \cap D: A \text { is locally pluriregular at } a\} .
$$

If $A \subset D$ is non locally pluripolar, then a classical result of Bedford and Taylor (see $[6,7]$ ) says that $A^{*}$ is locally pluriregular and $A \backslash A^{*}$ is locally pluripolar. Moreover, $A^{*}$ is locally of type $\mathcal{G}_{\delta}$, that is, for every $a \in A^{*}$ there is an open

${ }^{1}$ Observe that this function depends on the system of approach regions. 
neighborhood $U \subset D$ of $a$ such that $A^{*} \cap U$ is a countable intersection of open sets.

Now we are in the position to formulate the following version of the plurisubharmonic measure.

Definition 2.3. For a set $A \subset \bar{D}$, let $\widetilde{A}=\widetilde{A}(\mathcal{A}):=\bigcup_{P \in \mathcal{E}(A)} P$, where

$$
\mathcal{E}(A)=\mathcal{E}(A, \mathcal{A}):=\left\{P \subset \bar{D}: P \text { is locally pluriregular, } \bar{P} \subset A^{*}\right\},
$$

The plurisubharmonic measure of $A$ relative to $D$ is the function $\widetilde{\omega}(\cdot, A, D)$ defined by

$$
\widetilde{\omega}(z, A, D):=\omega(z, \widetilde{A}, D), \quad z \in D .
$$

It is worthy to remark that $\widetilde{\omega}(\cdot, A, D) \in \mathcal{P} \mathcal{S H}(D)$ and $0 \leq \widetilde{\omega}(z, A, D) \leq 1, z \in$ D. Moreover,

$$
(\mathcal{A}-\lim \sup \widetilde{\omega}(\cdot, A, D))(z)=0, \quad z \in \widetilde{A} .
$$

An example in [3] shows that in general, $\omega(\cdot, A, D) \neq \widetilde{\omega}(\cdot, A, D)$ on $D$. Section 10 below is devoted to the study of $\widetilde{\omega}(\cdot, A, D)$ in some important cases.

Now we compare the plurisubharmonic measure $\widetilde{\omega}(\cdot, A, D)$ with Siciak's relative extremal function $\omega(\cdot, A, D)$. We only consider two important special cases: $A \subset D$ and $A \subset \partial D$. For the moment, we only focus on the case where $A \subset D$. The latter one will be discussed in Section 10 below.

If $A$ is an open subset of an arbitrary complex manifold $D$, then it is easy to see that

$$
\widetilde{\omega}(z, A, D)=\omega(z, A, D), \quad z \in D .
$$

If $A$ is a (not necessarily open) subset of an arbitrary complex manifold $D$, then we will prove in Proposition 7.1 below that

$$
\widetilde{\omega}(z, A, D)=\omega\left(z, A^{*}, D\right), \quad z \in D .
$$

On the other hand, if, morever, $D$ is a bounded open subset of $\mathbb{C}^{n}$ then we have (see, for example, Lemma 3.5.3 in [18]) $\omega(z, A, D)=\omega\left(z, A^{*}, D\right), z \in D$. Consequently, under the last assumption,

$$
\widetilde{\omega}(z, A, D)=\omega(z, A, D), \quad z \in D .
$$

Our discussion shows that at least in the case where $A \subset D$, the notion of the plurisubharmonic measure is a good candidate for generalizing Siciak's relative extremal function to the manifold context in the theory of separate holomorphy.

For a good background of the pluripotential theory, see the books [18] or [20].

\subsection{Examples of systems of approach regions}

There are many systems of approach regions which are very useful in Complex Analysis. In this subsection we present some of them. 


\subsubsection{Canonical system of approach regions}

It has been given by Definition 2.1 (i)-(ii').

2.2.2. System of angular (or Stolz) approach regions for the open unit disc

Let $E$ be the open unit disc of $\mathbb{C}$. Put

$$
\mathcal{A}_{\alpha}(\zeta):=\left\{t \in E:\left|\arg \left(\frac{\zeta-t}{\zeta}\right)\right|<\alpha\right\}, \quad \zeta \in \partial E, 0<\alpha<\frac{\pi}{2},
$$

where $\arg : \mathbb{C} \rightarrow(-\pi, \pi]$ is as usual the argument function. $\mathcal{A}=\left(\mathcal{A}_{\alpha}(\zeta)\right)_{\zeta \in \partial E, 0<\alpha<\frac{\pi}{2}}$ is referred to as the system of angular (or Stolz) approach regions for E. In this context $\mathcal{A}-\lim$ is also called angular limit.

2.2.3. System of angular approach regions for certain "good" open subsets of Riemann surfaces

Now we generalize the previous construction (for the open unit disc) to a global situation. More precisely, we will use as the local model the system of angular approach regions for $E$. Let $X$ be a complex manifold of dimension 1, in other words, $X$ is a Riemann surface, and $D \subset X$ an open set. Then $D$ is said to be good at a point $\zeta \in \partial D^{2}$ if there is a Jordan domain $U \subset X$ such that $\zeta \in U$ and $U \cap \partial D$ is the interior of a Jordan curve.

Suppose that $D$ is good at $\zeta$. This point is said to be of type 1 if there is a neighborhood $V$ of $\zeta$ such that $V_{0}=V \cap D$ is a Jordan domain. Otherwise, $\zeta$ is said to be of type 2. We see easily that if $\zeta$ is of type 2, then there are an open neighborhood $V$ of $\zeta$ and two disjoint Jordan domains $V_{1}, V_{2}$ such that $V \cap D=$ $V_{1} \cup V_{2}$. Moreover, $D$ is said to be good on a subset $A$ of $\partial D$ if $D$ is good at all points of $A$.

Here is a simple example which may clarify the above definitions. Let $G$ be the open square in $\mathbb{C}$ with vertices $1+i,-1+i,-1-i$, and $1-i$. Define the domain

$$
D:=G \backslash\left[-\frac{1}{2}, \frac{1}{2}\right]
$$

Then $D$ is good on $\partial G \cup\left(-\frac{1}{2}, \frac{1}{2}\right)$. All points of $\partial G$ are of type 1 and all points of $\left(-\frac{1}{2}, \frac{1}{2}\right)$ are of type 2 .

Suppose now that $D$ is good on a nonempty subset $A$ of $\partial D$. We define the system of angular approach regions supported on $A: \mathcal{A}=\left(\mathcal{A}_{\alpha}(\zeta)\right)_{\zeta \in \bar{D}, \alpha \in I_{\zeta}}$ as follows:

${ }^{2}$ In the work [35] we use the more appealing word Jordan-curve-like for this notion. 
- If $\zeta \in \bar{D} \backslash A$, then $\left(\mathcal{A}_{\alpha}(\zeta)\right)_{\alpha \in I_{\zeta}}$ coincide with the canonical approach regions.

- If $\zeta \in A$, then by using a conformal mapping $\Phi$ from $V_{0}$ (respectively $V_{1}$ and $V_{2}$ ) onto $E$ when $\zeta$ is of type 1 (respectively 2 ), we can "transfer" the angular approach regions at the point $\Phi(\zeta) \in \partial E:\left(\mathcal{A}_{\alpha}(\Phi(\zeta))\right)_{0<\alpha<\frac{\pi}{2}}$ to those at the point $\zeta \in \partial D$ (see [35] for more detailed explanations).

Making use of conformal mappings in a local way, we can transfer, in the same way, many notions which exist on $E$ (respectively $\partial E$ ) to those on $D$ (respectively $\partial D$ ).

\subsubsection{System of conical approach regions}

Let $D \subset \mathbb{C}^{n}$ be a domain and $A \subset \partial D$. Suppose in addition that for every point $\zeta \in A$ there exists the (real) tangent space $T_{\zeta}$ to $\partial D$ at $\zeta$. We define the system of conical approach regions supported on $A: \mathcal{A}=\left(\mathcal{A}_{\alpha}(\zeta)\right)_{\zeta \in \bar{D}, \alpha \in I_{\zeta}}$ as follows:

- If $\zeta \in \bar{D} \backslash A$, then $\left(\mathcal{A}_{\alpha}(\zeta)\right)_{\alpha \in I_{\zeta}}$ coincide with the canonical approach regions.

- If $\zeta \in A$, then

$$
\mathcal{A}_{\alpha}(\zeta):=\left\{z \in D:|z-\zeta|<\alpha \cdot \operatorname{dist}\left(z, T_{\zeta}\right)\right\},
$$

where $I_{\zeta}:=(1, \infty)$ and $\operatorname{dist}\left(z, T_{\zeta}\right)$ denotes the Euclidean distance from the point $z$ to $T_{\zeta}$.

We can also generalize the previous construction to a global situation:

$X$ is an arbitrary complex manifold, $D \subset X$ is an open set and $A \subset \partial D$ is a subset with the property that at every point $\zeta \in A$ there exists the (real) tangent space $T_{\zeta}$ to $\partial D$.

We can also formulate the notion of points of type 1 or 2 in this general context in the same way as we have already done in Paragraph 3 above.

\subsection{Cross and separate holomorphicity and $\mathcal{A}$-limit}

Let $X, Y$ be two complex manifolds, let $D \subset X, G \subset Y$ be two nonempty open sets, let $A \subset \bar{D}$ and $B \subset \bar{G}$. Moreover, $D$ (respectively $G$ ) is equipped with a system of approach regions $\mathcal{A}(D)=\left(\mathcal{A}_{\alpha}(\zeta)\right)_{\zeta \in \bar{D}, \alpha \in I_{\zeta}}$ (respectively $\mathcal{A}(G)=$ $\left(\mathcal{A}_{\alpha}(\eta)\right)_{\eta \in \bar{G}, \alpha \in I_{\eta}}$ ). We define a 2-fold cross $W$, its interior $W^{\mathrm{o}}$ and its regular part $\widetilde{W}$ (with respect to $\mathcal{A}(D)$ and $\mathcal{A}(G)$ ) as

$$
\begin{aligned}
W & =\mathbb{X}(A, B ; D, G):=((D \cup A) \times B) \bigcup(A \times(G \cup B)), \\
W^{\mathrm{o}} & =\mathbb{X}^{\mathrm{O}}(A, B ; D, G):=(D \times B) \cup(A \times G), \\
\widetilde{W} & =\widetilde{\mathbb{X}}(A, B ; D, G):=((D \cup \widetilde{A}) \times \widetilde{B}) \bigcup(\widetilde{A} \times(G \cup \widetilde{B})) .
\end{aligned}
$$

Moreover, put

$$
\begin{array}{lll}
\omega(z, w):=\omega(z, A, D)+\omega(w, B, G), & & (z, w) \in D \times G, \\
\widetilde{\omega}(z, w):=\widetilde{\omega}(z, A, D)+\widetilde{\omega}(w, B, G), & & (z, w) \in D \times G .
\end{array}
$$


For a 2-fold cross $W:=\mathbb{X}(A, B ; D, G)$ let

$$
\begin{aligned}
& \widehat{W}:=\widehat{\mathbb{X}}(A, B ; D, G)=\{(z, w) \in D \times G: \omega(z, w)<1\}, \\
& \widehat{\widetilde{W}}:=\widehat{\mathbb{X}}(\widetilde{A}, \widetilde{B} ; D, G)=\{(z, w) \in D \times G: \widetilde{\omega}(z, w)<1\} .
\end{aligned}
$$

Let $Z$ be a complex analytic space. We say that a mapping $f: W^{\mathrm{o}} \longrightarrow Z$ is separately holomorphic and write $f \in \mathcal{O}_{s}\left(W^{\circ}, Z\right)$, if, for any $a \in A$ (respectively $b \in B$ ) the restricted mapping $f(a, \cdot)$ (respectively $f(\cdot, b)$ ) is holomorphic on $G$ (respectively on $D$ ).

We say that a mapping $f: W \longrightarrow Z$ is separately continuous and write $f \in \mathcal{C}_{s}(W, Z)$ if, for any $a \in A$ (respectively $b \in B$ ) the restricted mapping $f(a, \cdot)$ (respectively $f(\cdot, b)$ ) is continuous on $G \cup B$ (respectively on $D \cup A$ ).

In virtue of (2.1), for every $(\zeta, \eta) \in \widetilde{W}$ and every $\alpha \in I_{\zeta}, \beta \in I_{\eta}$, there are open neighborhoods $U$ of $\zeta$ and $V$ of $\eta$ such that

$$
\left(U \cap \mathcal{A}_{\alpha}(\zeta)\right) \times\left(V \cap \mathcal{A}_{\beta}(\eta)\right) \subset \widehat{\widetilde{W}}
$$

Then a mapping $f: \widehat{\widetilde{W}} \longrightarrow Z$ is said to admit $\mathcal{A}$-limit $\lambda$ at $(\zeta, \eta) \in \widetilde{W}$, and one writes

$$
(\mathcal{A}-\lim f)(\zeta, \eta)=\lambda,^{3}
$$

if, for all $\alpha \in I_{\zeta}, \beta \in I_{\eta}$,

$$
\lim _{\widehat{\widetilde{W}} \ni(z, w) \rightarrow(\zeta, \eta), z \in \mathcal{A}_{\alpha}(\zeta), w \in \mathcal{A}_{\beta}(\eta)} f(z, w)=\lambda .
$$

Throughout the paper, for a topological space $\mathcal{M}, \mathcal{C}(\mathcal{M}, Z)$ denotes the set of all continuous mappings $f: \mathcal{M} \longrightarrow Z$. If, moreover, $Z=\mathbb{C}$, then $\mathcal{C}(\mathcal{M}, \mathbb{C})$ is equipped with the "sup-norm" $|f|_{\mathcal{M}}:=\sup _{\mathcal{M}}|f| \in[0, \infty]$. A mapping $f$ : $\mathcal{M} \longrightarrow Z$ is said to be bounded if there exist an open neighborhood $U$ of $f(\mathcal{M})$ in $Z$ and a holomorphic embedding $\phi$ of $U$ into a bounded polydisc of $\mathbb{C}^{k}$ such that $\phi(U)$ is an analytic set in this polydisc. $f$ is said to be locally bounded along $\mathcal{N} \subset \mathcal{M}$ if for every point $z \in \mathcal{N}$, there is an open neighborhood $U$ of $z$ (in $\mathcal{M}$ ) such that $\left.f\right|_{U}: U \longrightarrow Z$ is bounded. $f$ is said to be locally bounded if it is so for $\mathcal{N}=\mathcal{M}$. It is clear that if $Z=\mathbb{C}$ then the above notions of boundedness coincide with the usual ones.

\subsection{Hartogs extension property}

The following example (see Shiffman [43]) shows that an additional hypothesis on the "target space" $Z$ is necessary in order that the Problem 1.1 makes sense.

${ }^{3}$ Note that here $\mathcal{A}=\mathcal{A}(D) \times \mathcal{A}(G)$. 
Consider the mapping $f: \mathbb{C}^{2} \longrightarrow \mathbb{P}^{1}$ given by

$$
f(z, w):= \begin{cases}{\left[(z+w)^{2}:(z-w)^{2}\right],} & (z, w) \neq(0,0), \\ {[1: 1],} & (z, w)=(0,0) .\end{cases}
$$

Then $f \in \mathcal{O}_{s}\left(\mathbb{X}^{\mathrm{o}}(\mathbb{C}, \mathbb{C} ; \mathbb{C}, \mathbb{C}), \mathbb{P}^{1}\right)$, but $f$ is not continuous at $(0,0)$.

We recall here the following notion (see, for example, Shiffman [42]). Let $p \geq 2$ be an integer. For $0<r<1$, the Hartogs figure in dimension $p$, denoted by $H_{p}(r)$, is given by

$$
H_{p}(r):=\left\{\left(z^{\prime}, z_{p}\right) \in E^{p}:\left\|z^{\prime}\right\|<r \text { or }\left|z_{p}\right|>1-r\right\},
$$

where $E$ is the open unit disc of $\mathbb{C}$ and $z^{\prime}=\left(z_{1}, \ldots, z_{p-1}\right),\left\|z^{\prime}\right\|:=\max _{1 \leq j \leq p-1}\left|z_{j}\right|$.

Definition 2.4. A complex analytic space $Z$ is said to possess the Hartogs extension property in dimension $p$ if every mapping $f \in \mathcal{O}\left(H_{p}(r), Z\right)$ extends to a mapping $\hat{f} \in \mathcal{O}\left(E^{p}, Z\right)$. Moreover, $Z$ is said to possess the Hartogs extension property if it does in any dimension $p \geq 2$.

It is a classical result of Ivashkovich (see [17]) that if $Z$ possesses the Hartogs extension property in dimension 2 , then it does in all dimensions $p \geq 2$. Some typical examples of complex analytic spaces possessing the Hartogs extension property are the complex Lie groups (see [4]), the taut spaces (see [49]), the Hermitian manifold with negative holomorphic sectional curvature (see [42]), the holomorphically convex Kähler manifold without rational curves (see [17]).

Here we mention an important characterization due to Shiffman (see [42]).

Theorem 2.5. A complex analytic space $Z$ possesses the Hartogs extension property if and only if for every domain $D$ of any Stein manifold $\mathcal{M}$, every mapping $f \in \mathcal{O}(D, Z)$ extends to a mapping $\hat{f} \in \mathcal{O}(\widehat{D}, Z)$, where $\widehat{D}$ is the envelope of holomorphy of $D$.

In the light of Definition 2.4 and Shiffman's theorem, the natural "target spaces" $Z$ for obtaining satisfactory answers to the Problem 1.1 are the complex analytic spaces which possess the Hartogs extension property.

\subsection{Statement of the main results}

We are now ready to state the main results.

Theorem A. Let $X, Y$ be two complex manifolds, let $D \subset X, G \subset Y$ be two open sets, let $A$ (respectively $B$ ) be a subset of $\bar{D}$ (respectively $\bar{G}$ ). $D$ (respectively $G)$ is equipped with a system of approach regions $\left(\mathcal{A}_{\alpha}(\zeta)\right)_{\zeta \in \bar{D}, \alpha \in I_{\zeta}}$ (respectively $\left.\left(\mathcal{A}_{\beta}(\eta)\right)_{\eta \in \bar{G}}, \beta \in I_{\eta}\right)$. Let $Z$ be a complex analytic space possessing the Hartogs extension property. Then, for every mapping $f: W \longrightarrow Z$ which satisfies the following conditions: 
- $f \in \mathcal{C}_{s}(W, Z) \cap \mathcal{O}_{s}\left(W^{\mathrm{o}}, Z\right)$;

- $f$ is locally bounded along $\mathbb{X}(A \cap \partial D, B \cap \partial G ; D, G) ;{ }^{4}$

- $\left.f\right|_{A \times B}$ is continuous at all points of $(A \cap \partial D) \times(B \cap \partial G)$,

there exists a unique mapping $\hat{f} \in \mathcal{O}(\widehat{\widetilde{W}}, Z)$ which admits $\mathcal{A}$-limit $f(\zeta, \eta)$ at every point $(\zeta, \eta) \in W \cap \widetilde{W}$.

If, moreover, $Z=\mathbb{C}$ and $|f|_{W}<\infty$, then

$$
|\hat{f}(z, w)| \leq|f|_{A \times B}^{1-\widetilde{\omega}(z, w)}|f|_{W}^{\widetilde{\omega}(z, w)}, \quad(z, w) \in \widetilde{\widetilde{W}}
$$

Theorem A has an important corollary. Before stating this, we need to introduce a terminology. A complex manifold $\mathcal{M}$ is said to be a Liouville manifold if $\mathcal{P S H}(\mathcal{M})$ does not contain any non-constant bounded above functions. We see clearly that the class of Liouville manifolds contains the class of connected compact manifolds.

Corollary B. We keep the hypothesis and the notation in Theorem A. Suppose in addition that $G$ is a Liouville manifold and that $\widetilde{A}, \widetilde{B} \neq \varnothing$. Then, for every mapping $f: W \longrightarrow Z$ which satisfies the following conditions:

- $f \in \mathcal{C}_{S}(W, Z) \cap \mathcal{O}_{s}\left(W^{\mathrm{o}}, Z\right)$;

- $f$ is locally bounded along $\mathbb{X}(A \cap \partial D, B \cap \partial G ; D, G)$;

- $\left.f\right|_{A \times B}$ is continuous at all points of $(A \cap \partial D) \times(B \cap \partial G)$,

there is a unique mapping $\hat{f} \in \mathcal{O}(D \times G, Z)$ which admits $\mathcal{A}$-limit $f(\zeta, \eta)$ at every point $(\zeta, \eta) \in W \cap \widetilde{W}$.

Corollary B follows immediately from Theorem A since $\widetilde{\omega}(\cdot, B, G) \equiv 0$.

We will see in Section 10 below that Theorem A and Corollary B generalize all the results discussed in Section 1 above. Moreover, they also give many new results. Although our main results have been stated only for the case of a 2-fold cross, they can be formulated for the general case of an $N$-fold cross with $N \geq 2$ (see also $[28,34])$.

\section{Holomorphic discs and a two-constant theorem}

We recall here some elements of Poletsky theory of discs, some background of the pluripotential theory and auxiliary results needed for the proof of Theorem A.

${ }^{4}$ It follows from Subsection 2.3 that

$$
\mathbb{X}(A \cap \partial D, B \cap \partial G ; D, G)=((D \cup A) \times(B \cap \partial G)) \bigcup((A \cap \partial D) \times(G \cup B)) .
$$




\subsection{Poletsky theory of discs and Rosay theorem on holomorphic dises}

Let $E$ denote as usual the open unit disc in $\mathbb{C}$. For a complex manifold $\mathcal{M}$, let $\mathcal{O}(\bar{E}, \mathcal{M})$ denote the set of all holomorphic mappings $\phi: E \longrightarrow \mathcal{M}$ which extend holomorphically to a neighborhood of $\bar{E}$. Such a mapping $\phi$ is called a holomorphic disc on $\mathcal{M}$. Moreover, for a subset $A$ of $\mathcal{M}$, let

$$
1_{A, \mathcal{M}}(z):= \begin{cases}1, & z \in A \\ 0, & z \in \mathcal{M} \backslash A .\end{cases}
$$

In the work [41] Rosay proved the following remarkable result.

Theorem 3.1. Let $u$ be an upper semicontinuous function on a complex manifold $\mathcal{M}$. Then the Poisson functional of $u$ defined by

$$
\mathcal{P}[u](z):=\inf \left\{\frac{1}{2 \pi} \int_{0}^{2 \pi} u\left(\phi\left(e^{i \theta}\right)\right) d \theta: \phi \in \mathcal{O}(\bar{E}, \mathcal{M}), \phi(0)=z\right\},
$$

is plurisubharmonic on $\mathcal{M}$.

Rosay theorem may be viewed as an important development in Poletsky theory of discs. Observe that special cases of Theorem 3.1 have been considered by Poletsky (see [38,39]), Lárusson-Sigurdsson (see [22]) and Edigarian (see [10]).

The following Rosay type result gives the connections between the Poisson functional of the characteristic function $1_{\mathcal{M} \backslash A, \mathcal{M}}$ and holomorphic discs.

Lemma 3.2. Let $\mathcal{M}$ be a complex manifold and let $A$ be a nonempty open subset of $\mathcal{M}$. Then for any $\epsilon>0$ and any $z_{0} \in \mathcal{M}$, there are an open neighborhood $U$ of $z_{0}$, an open subset $T$ of $\mathbb{C}$, and a family of holomorphic discs $\left(\phi_{z}\right)_{z \in U} \subset \mathcal{O}(\bar{E}, \mathcal{M})$ with the following properties:

(i) $\Phi \in \mathcal{O}(U \times E, \mathcal{M})$, where $\Phi(z, t):=\phi_{z}(t),(z, t) \in U \times E$;

(ii) $\phi_{z}(0)=z, \quad z \in U$;

(iii) $\phi_{z}(t) \in A, \quad t \in T \cap \bar{E}, z \in U$;

(iv) $\frac{1}{2 \pi} \int_{0}^{2 \pi} 1_{\partial E \backslash T, \partial E}\left(e^{i \theta}\right) d \theta<\mathcal{P}\left[1_{\mathcal{M} \backslash A, \mathcal{M}}\right]\left(z_{0}\right)+\epsilon$.

Proof. See Lemma 3.2 in [28].

The next result describes the situation in dimension 1 . It will be very useful later on.

Lemma 3.3. Let $T$ be an open subset of $\bar{E}$. Then

$$
\omega(0, T \cap E, E) \leq \frac{1}{2 \pi} \int_{0}^{2 \pi} 1_{\partial E \backslash T, T}\left(e^{i \theta}\right) d \theta .
$$


Proof. See, for example, Lemma 3.3 in [28].

The last result, which is an important consequence of Rosay's theorem, gives the connection between the Poisson functional and the plurisubharmonic measure.

Proposition 3.4. Let $\mathcal{M}$ be a complex manifold and $A$ a nonempty open subset of $\mathcal{M}$. Then $\omega(z, A, \mathcal{M})=\mathcal{P}\left[1_{\mathcal{M} \backslash A, \mathcal{M}}\right](z), z \in \mathcal{M}$.

Proof. See, for example, the proof of Proposition 3.4 in [28].

\subsection{Level sets of the relative extremal functions and a two-constant theorem}

Let $X$ be a complex manifold and $D \subset X$ an open set. Suppose that $D$ is equipped with a system of approach regions $\mathcal{A}=\left(\mathcal{A}_{\alpha}(\zeta)\right)_{\zeta \in \bar{D}, \alpha \in I_{\zeta}}$. For every open subset $G$ of $D$, there is a natural system of approach regions for $G$ which is called the induced system of approach regions $\mathcal{A}^{\prime}=\left(\mathcal{A}_{\alpha}^{\prime}(\zeta)\right)_{\zeta \in \bar{G}, \alpha \in I_{\zeta}^{\prime}}$ of $\mathcal{A}$ onto $G$. It is given by

$$
\mathcal{A}_{\alpha}^{\prime}(\zeta):=\mathcal{A}_{\alpha}(\zeta) \cap G, \quad \zeta \in \bar{G}, \alpha \in I_{\zeta}^{\prime},
$$

where $I_{\zeta}^{\prime}:=\left\{\alpha \in I_{\zeta}: \zeta \in \overline{\mathcal{A}_{\alpha}(\zeta) \cap G}\right\}$.

Proposition 3.5. Under the above hypothesis and notation, let $A \subset \bar{D}$ be a locally pluriregular set (relative to $\mathcal{A}$ ). For $0<\delta<1$, define the $\delta$-level set of $D$ relative to A as follows

$$
D_{\delta, A}:=\{z \in D: \omega(z, A, D)<1-\delta\} .
$$

We equip $D_{\delta, A}$ with the induced system of approach regions $\mathcal{A}^{\prime}$ of $\mathcal{A}$ onto $D_{\delta, A}$ (see Subsection 2.1 above). Then $A \subset \overline{D_{\delta, A}}$ and

$$
\omega\left(z, A, D_{\delta, A}\right)=\frac{\omega(z, A, D)}{1-\delta}, \quad z \in D_{\delta, A} .
$$

Moreover, A is locally pluriregular relative to $\mathcal{A}^{\prime}$.

Proof. Since $A$ is locally pluriregular, we see that

$$
(\mathcal{A}-\lim \sup \omega(\cdot, A, D))(z)=0, \quad z \in A .
$$

Therefore, for every $z \in A$ and $\alpha \in I_{z}$, there is an open neighborhood $U$ of $z$ such that $\varnothing \neq \mathcal{A}_{\alpha}(z) \cap U \subset D_{\delta, A}$. Hence, $A \subset \overline{D_{\delta, A}}$.

Next, we turn to the proof of identity (3.1). Observe that $0 \leq \frac{\omega(\cdot, A, D)}{1-\delta} \leq 1$ on $D_{\delta, A}$ by definition. This, combined with (3.2), implies that

$$
\frac{\omega(z, A, D)}{1-\delta} \leq \omega\left(z, A, D_{\delta, A}\right), \quad z \in D_{\delta, A} .
$$


To prove the converse inequality of (3.3), let $u \in \mathcal{P} \mathcal{S H}\left(D_{\delta, A}\right)$ be such that $u \leq 1$ on $D_{\delta, A}$ and $\mathcal{A}^{\prime}-\lim \sup u \leq 0$ on $A$. Consider the following function

$$
\hat{u}(z):= \begin{cases}\max \{(1-\delta) u(z), \omega(z, A, D)\}, & z \in D_{\delta, A}, \\ \omega(z, A, D), & z \in D \backslash D_{\delta, A} .\end{cases}
$$

It can be checked that $\hat{u} \in \mathcal{P S H}(D)$ and $0 \leq \hat{u} \leq 1$. Moreover, in virtue of the assumption on $u$ and (3.2) and (3.4), we have that

$$
\begin{aligned}
& (\mathcal{A}-\lim \sup \hat{u})(a) \\
& \leq \max \left\{(1-\delta)\left(\mathcal{A}^{\prime}-\lim \sup u\right)(a),(\mathcal{A}-\lim \sup \omega(\cdot, A, D))(a)\right\}=0
\end{aligned}
$$

for all $a \in A$. Consequently, $\hat{u} \leq \omega(\cdot, A, D)$. In particular, one gets from (3.4) that

$$
u(z) \leq \frac{\omega(z, A, D)}{1-\delta}, \quad z \in D_{\delta, A} .
$$

Since $u$ is arbitrary, we deduce from the latter estimate that the converse inequality of (3.3) also holds. This, combined with (3.3), completes the proof of (3.1).

To prove the last conclusion of the proposition, fix a point $a \in A$ and an open neighborhood $U$ of $a$. Then we have

$$
\begin{aligned}
& \left(\mathcal{A}-\lim \sup \omega\left(\cdot, A \cap U, D_{\delta, A} \cap U\right)\right)(a) \\
& \leq\left(\mathcal{A}-\lim \sup \omega\left(\cdot, A \cap U,(D \cap U)_{\delta, A \cap U}\right)(a)\right. \\
& =\frac{1}{1-\delta} \cdot(\mathcal{A}-\lim \sup \omega(\cdot, A \cap U, D \cap U))(a)=0,
\end{aligned}
$$

where the first equality follows from identity (3.1) and the second one from the hypothesis that $A$ is locally pluriregular.

The following two-constant theorem for plurisubharmonic functions will play an important role in the proof of the estimate in Theorem A.

Theorem 3.6. Let $X$ be a complex manifold and $D \subset X$ an open subset. Suppose that $D$ is equipped with a system of approach regions $\left(\mathcal{A}_{\alpha}(\zeta)\right)_{\zeta \in \bar{D}, \alpha \in I_{\zeta}}$. Let $A \subset \bar{D}$ be a locally pluriregular set. Let $m, M \in \mathbb{R}$ and $u \in \mathcal{P S H}(D)$ such that $u(z) \leq M$ for $z \in D$, and $(\mathcal{A}-\lim \sup u)(z) \leq m$ for $z \in A$. Then

$$
u(z) \leq m(1-\omega(z, A, D))+M \cdot \omega(z, A, D), \quad z \in D .
$$

Proof. It follows immediately from Definition 2.2.

Theorem 3.7. We keep the hypotheses and notation of Theorem 3.6. Let $f$ be a bounded function in $\mathcal{O}(D, \mathbb{C})$ such that $(\mathcal{A}-\lim f)(\zeta)=0, \zeta \in A$. Then $f(z)=0$ for all $z \in D$ such that $\omega(z, A, D) \neq 1$.

Proof. Fix a finite positive constant $M$ such that $|f|_{D}<M$. Consequently, the desired conclusion follows from applying Theorem 3.6 to the function $u:=$ $\log |f|$. 


\subsection{Construction of discs}

In this subsection we present the construction of discs à la Poletsky (see [39]). This is one of the main ingredients in the proof of Theorem A.

Let mes denote the Lebesgue measure on the unit circle $\partial E$. For a bounded mapping $\phi \in \mathcal{O}\left(E, \mathbb{C}^{n}\right)$ and $\zeta \in \partial E, f(\zeta)$ denotes the angular limit value of $f$ at $\zeta$ if it exists. A classical theorem of Fatou says that mes $(\{\zeta \in \partial E: \exists f(\zeta)\})=2 \pi$. For $z \in \mathbb{C}^{n}$ and $r>0$, let $\mathbb{B}(z, r)$ denote the open ball centered at $z$ with radius $r$.

Theorem 3.8. Let $D$ be a bounded open set in $\mathbb{C}^{n}, A \subset \bar{D}, z_{0} \in D$ and $\epsilon>0$. Let $\mathcal{A}$ be a system of approach regions for $D$. Suppose in addition that $A$ is locally pluriregular (relative to $\mathcal{A}$ ). Then there exist a bounded mapping $\phi \in \mathcal{O}\left(E, \mathbb{C}^{n}\right)$ and a measurable subset $\Gamma_{0} \subset \partial E$ with the following properties:

1) $\Gamma_{0}$ is pluriregular (with respect to the system of angular approach regions), $\phi(0)=z_{0}, \phi(E) \subset \bar{D}, \Gamma_{0} \subset\{\zeta \in \partial E: \phi(\zeta) \in \bar{A}\}$, and

$$
1-\frac{1}{2 \pi} \cdot \operatorname{mes}\left(\Gamma_{0}\right)<\omega\left(z_{0}, A, D\right)+\epsilon .
$$

2) Let $f \in \mathcal{C}(D \cup \bar{A}, \mathbb{C}) \cap \mathcal{O}(D, \mathbb{C})$ be such that $f(D)$ is bounded. Then there exist a bounded function $g \in \mathcal{O}(E, \mathbb{C})$ such that $g=f \circ \phi$ in a neighborhood of $0 \in E$ and ${ }^{5} g(\zeta)=(f \circ \phi)(\zeta)$ for all $\zeta \in \Gamma_{0}$. Moreover, $\left.g\right|_{\Gamma_{0}} \in \mathcal{C}\left(\Gamma_{0}, \mathbb{C}\right)$.

This theorem motivates the following

Definition 3.9. We keep the hypothesis and notation of Theorem 3.8. Then every pair $\left(\phi, \Gamma_{0}\right)$ satisfying the conclusions 1$\left.)-2\right)$ of this theorem is said to be an $\epsilon$ candidate for the triplet $\left(z_{0}, A, D\right)$.

Theorem 3.8 says that there always exist $\epsilon$-candidates for all triplets $(z, A, D)$.

Proof. First we will construct $\phi$. To do this we will construct by induction a sequence $\left(\phi_{k}\right)_{k=1}^{\infty} \subset \mathcal{O}(\bar{E}, D)$ which approximates $\phi$ as $k \nearrow \infty$. This will allow to define the desired mapping as $\phi:=\lim _{k \rightarrow \infty} \phi_{k}$. The construction of such a sequence is divided into three steps.

For $0<\delta, r<1$ let

$$
\begin{aligned}
D_{a, r} & :=D \cap \mathbb{B}(a, r), \quad a \in A . \\
A_{a, r, \delta} & :=\left\{z \in D_{a, r}: \omega\left(z, A \cap \mathbb{B}(a, r), D_{a, r}\right)<\delta\right\}, \quad a \in A, \\
A_{r, \delta} & :=\bigcup_{a \in A} A_{a, r, \delta},
\end{aligned}
$$

where in the second ":=" $D_{a, r}$ is equipped with the induced system of approach regions of $\mathcal{A}$ onto $D_{a, r}$ (see Subsection 3.2 above).

5 Note here that by Part 1$),(f \circ \phi)(\zeta)$ exists for all $\zeta \in \Gamma_{0}$. 
Suppose without loss of generality that $D \subset \mathbb{B}(0,1)$.

Step 1. Construction of $\phi_{1}$

Let $\delta_{0}:=\frac{\epsilon}{3}$ and $r_{0}:=1$. Fix $0<\delta_{1}<\frac{\delta_{0}}{3}$ and $0<r_{1}<\frac{r_{0}}{3}$. Applying Proposition 3.4, we obtain $\phi_{1} \in \mathcal{O}(\bar{E}, D)$ such that $\phi_{1}(0)=z_{0}$ and

$$
1-\frac{1}{2 \pi} \cdot \operatorname{mes}\left(\partial E \cap \phi_{1}^{-1}\left(A_{r_{1}, \delta_{1}}\right)\right) \leq \omega\left(z_{0}, A_{r_{1}, \delta_{1}}, D\right)+\delta_{0} .
$$

On the other hand, using (3.5) and Definition 2.2 and the hypothesis that $A$ is locally pluriregular, we obtain

$$
\omega\left(z_{0}, A_{r_{1}, \delta_{1}}, D\right) \leq \omega\left(z_{0}, A, D\right) .
$$

Consequently, we may choose a subset $\Gamma_{1}$ of $\Gamma_{0}:=\partial E \cap \phi_{1}^{-1}\left(A_{r_{1}, \delta_{1}}\right)$ which consists of finite disjoint closed arcs $\left(\Gamma_{1 j}\right)_{j \in J_{1}}$ so that

$$
1-\frac{1}{2 \pi} \cdot \operatorname{mes}\left(\Gamma_{1}\right)<\omega\left(z_{0}, A_{r_{1}, \delta_{1}}, D\right)+2 \delta_{0} \leq \omega\left(z_{0}, A, D\right)+2 \delta_{0},
$$

and

$$
\sup _{t, \tau \in \Gamma_{1 j}}|t-\tau|<2 \delta_{1}, \sup _{t, \tau \in \Gamma_{1 j}}\left|\phi_{1}(t)-\phi_{1}(\tau)\right|<2 r_{1}, \quad j \in J_{1} .
$$

Step 2. Construction of $\phi_{k+1}$ from $\phi_{k}$ for all $k \geq 1$

By the inductive construction we have $0<\delta_{k}<\frac{\delta_{k-1}}{3}$ and $0<r_{k}<\frac{r_{k-1}}{3}$ and $\phi_{k} \in \mathcal{O}(\bar{E}, D)$ such that $\phi_{k}(0)=z_{0}$ and there exists a closed subset $\Gamma_{k}$ of $\partial E \cap$ $\phi_{k}^{-1}\left(A_{r_{k}, \delta_{k}}\right) \cap \Gamma_{k-1}$ which consists of finite closed $\operatorname{arcs}\left(\Gamma_{k, j}\right)_{j \in J_{k}}$ such that $\Gamma_{k}$ is relatively compact in the interior of $\Gamma_{k-1}$, and

$$
1-\frac{1}{2 \pi} \cdot \operatorname{mes}\left(\Gamma_{k}\right)<1-\frac{1}{2 \pi} \cdot \operatorname{mes}\left(\Gamma_{k-1}\right)+2 \delta_{k-1},
$$

and

$$
\sup _{t, \tau \in \Gamma_{k, j}}|t-\tau|<2 \delta_{k}, \sup _{t, \tau \in \Gamma_{k, j}}\left|\phi_{k}(t)-\phi_{k}(\tau)\right|<2 r_{k}, \quad j \in J_{k},
$$

and

$$
\left|\phi_{k}-\phi_{k-1}\right|_{\Gamma_{k}}<2 r_{k-1} .
$$

Here we make the convention that the last inequality is empty when $k=1$.

In particular, we have that $\phi_{k}\left(\Gamma_{k}\right) \subset A_{r_{k}, \delta_{k}}$. Therefore, by (3.5), for every $\zeta \in \phi_{k}\left(\Gamma_{k}\right)$ there is $a \in A$ such that $\zeta \in A_{a, r_{k}, \delta_{k}}$, that is,

$$
\omega\left(\zeta, A \cap \mathbb{B}\left(a, r_{k}\right), D_{a, r_{k}}\right)<\delta_{k} .
$$


Using the hypothesis that $A$ is locally pluriregular and (3.5) we see that

$$
\omega\left(z, A_{r, \delta} \cap D_{a, r_{k}}, D_{a, r_{k}}\right) \leq \omega\left(z, A \cap \mathbb{B}\left(a, r_{k}\right), D_{a, r_{k}}\right), \quad 0<\delta, r<1 .
$$

Consequently, for every $\zeta \in \phi_{k}\left(\Gamma_{k}\right)$ there is $a \in A$ such that

$$
\omega\left(\zeta, A_{r, \delta} \cap D_{a, r_{k}}, D_{a, r_{k}}\right)<\delta_{k}, \quad 0<\delta, r<1 .
$$

Using the last estimate and arguing as in [39, pages 120-121] (see also the proof of Theorem 1.10.7 in [19] for a nice presentation), we can choose $0<\delta_{k+1}<\frac{\delta_{k}}{3}$ and $0<r_{k+1}<\frac{r_{k}}{3}$ and $\phi_{k+1} \in \mathcal{O}(\bar{E}, D)$ such that $\phi_{k+1}(0)=z_{0}$, and there exists a closed subset $\Gamma_{k+1}$ of $\partial E \cap \phi_{k+1}^{-1}\left(A_{r_{k+1}, \delta_{k+1}}\right) \cap \Gamma_{k}$ which consists of finite closed arcs $\left(\Gamma_{k+1, j}\right)_{j \in J_{k+1}}$ such that $\Gamma_{k+1}$ is relatively compact in the interior of $\Gamma_{k}$, and

$$
1-\frac{1}{2 \pi} \cdot \operatorname{mes}\left(\Gamma_{k+1}\right)<1-\frac{1}{2 \pi} \cdot \operatorname{mes}\left(\Gamma_{k}\right)+2 \delta_{k},
$$

and

$$
\sup _{t, \tau \in \Gamma_{k+1, j}}|t-\tau|<2 \delta_{k+1}, \sup _{t, \tau \in \Gamma_{k+1, j}}\left|\phi_{k+1}(t)-\phi_{k+1}(\tau)\right|<2 r_{k+1}, \quad j \in J_{k+1},
$$

and

$$
\left|\phi_{k+1}-\phi_{k}\right|_{\Gamma_{k+1}}<2 r_{k} .
$$

Step 3. Construction of $\phi$ from the sequence $\left(\phi_{k}\right)_{k=1}^{\infty}$

In summary, we have constructed a decreasing sequence $\left(\Gamma_{k}\right)_{k=1}^{\infty}$ of closed subsets of $\partial E$. Consider the new closed set

$$
\Gamma:=\bigcap_{k=1}^{\infty} \Gamma_{k} .
$$

By (3.7)-(3.8),

$$
\frac{1}{2 \pi} \cdot \operatorname{mes}(\Gamma)=\frac{1}{2 \pi} \operatorname{mes}\left(\Gamma_{1}\right)-2 \sum_{k=1}^{\infty} \delta_{k}>\frac{1}{2 \pi} \operatorname{mes}\left(\Gamma_{1}\right)-3 \delta_{1} .
$$

This, combined with (3.6), implies the following property

(i) $1-\frac{1}{2 \pi} \cdot \operatorname{mes}(\Gamma)<1-d \operatorname{frac} 12 \pi \cdot \operatorname{mes}\left(\Gamma_{1}\right)+3 \delta_{1} \leq \omega\left(z_{0}, A, D\right)+2 \delta_{0}+3 \delta_{1}<$ $\omega\left(z_{0}, A, D\right)+\epsilon$.

On the other hand, we recall from the above construction the following properties:

(ii) $\phi_{k}(\Gamma) \subset \phi_{k}\left(\Gamma_{k}\right) \subset A_{r_{k}, \delta_{k}}$. 
(iii) $\delta_{0}=\frac{\epsilon}{3}, r_{0}=1,0<\delta_{k+1}<\frac{\delta_{k}}{3}, 0<r_{k+1}<\frac{r_{k}}{3}$ and $\left|\phi_{k+1}-\phi_{k}\right|_{\Gamma} \leq$ $\left|\phi_{k+1}-\phi_{k}\right|_{\Gamma_{k+1}}<2 r_{k}$.

(iv) $\sup _{t, \tau \in \Gamma_{k j}}|t-\tau|<2 \delta_{k}$ and $\sup _{t, \tau \in \Gamma_{k, j}}\left|\phi_{k}(t)-\phi_{k}(\tau)\right|<2 r_{k}, \quad j \in J_{k}$.

(v) For every $\zeta \in \Gamma$ there exists a sequence $\left(j_{k}\right)_{k \geq 1}$ such that $j_{k} \in J_{k}$, and $\zeta$ is an interior point of $\Gamma_{k, j_{k}}$, and $\Gamma_{k+1, j_{k+1}} \Subset \Gamma_{k, j_{k}}$, and $\zeta=\bigcap_{k=1}^{\infty} \Gamma_{k, j_{k}}$.

Therefore, we are able to apply the Khinchin-Ostrowski theorem (see [11, Theorem 4 , page 397]) to the sequence $\left(\phi_{k}\right)_{k=1}^{\infty}$. Consequently, this sequence converges uniformly on compact subsets of $E$ to a mapping $\phi \in \mathcal{O}(E, D)$. Moreover, $\phi$ admits (angular) boundary values at all points of $\Gamma$ and $\phi(\Gamma) \subset \bigcap_{k=1}^{\infty} A_{r_{k}, \delta_{k}} \subset \bar{A}$.

Observe that since $\phi_{k}(0)=\phi(0)=z_{0} \in D$ and $f \in \mathcal{C}(D \cup \bar{A}, \mathbb{C}) \cap \mathcal{O}(D, \mathbb{C})$, the sequence $\left(f \circ \phi_{k}\right)_{k=1}^{\infty}$ converges to $f \circ \phi$ uniformly on a neighborhood of $0 \in E$. On the other hand, $f(D)$ is bounded by the hypothesis. Thus by Montel theorem, the family $\left(f \circ \phi_{k}\right)_{k=1}^{\infty} \subset \mathcal{O}(E, \mathbb{C})$ is normal. Consequently, the sequence $(f \circ$ $\left.\phi_{k}\right)_{k=1}^{\infty}$ converges uniformly on compact subsets of $E$. Let $g$ be the limit mapping. Then $g \in \mathcal{O}(E, \mathbb{C})$ and $g=f \circ \phi$ in a neighborhood of $0 \in E$. Moreover, it follows from (i)-(iii) above and the hypothesis $f \in \mathcal{C}(D \cup \bar{A}, \mathbb{C})$ that $g(\zeta)=(f \circ \phi)(\zeta)$ for all $\zeta \in \Gamma$. We deduce from (iii)-(v) above that $\left.g\right|_{\Gamma} \in \mathcal{C}(\Gamma, \mathbb{C})$ Finally, applying Lemma 4.1 below we may choose a locally pluriregular subset $\Gamma_{0} \subset \Gamma$ (relative to the system of angular approach regions) such that mes $\left(\Gamma_{0}\right)=\operatorname{mes}(\Gamma)$. Hence, the proof is finished.

It is worthy to remark that $\phi(E) \subset \bar{D}$; but in general, $\phi(E) \not \subset D$ !

The last result of this section sharpens Theorem 3.8.

Theorem 3.10. Let $D$ be a bounded open set in $\mathbb{C}^{n}, A \subset \bar{D}$, and $\epsilon>0$. Let $\mathcal{A}$ be a system of approach regions for $D$. Suppose in addition that $A$ is locally pluriregular (relative to $\mathcal{A}$ ). Then there exists a Borel mapping $\Phi: D \times E \longrightarrow \mathbb{C}^{n}$ with the following property: for every $z \in D$, there is a measurable subset $\Gamma_{z}$ of $\partial E$ such that $\left(\Phi(z, \cdot), \Gamma_{z}\right)$ is an $\epsilon$-candidate for the triplet $(z, A, D)$.

Roughly speaking, this result says that one can construct $\epsilon$-candidates for $(z, A, D)$ so that they depend in a Borel-measurable way on $z \in D$.

Proof. Observe that in Proposition 3.4 we can construct $\epsilon$-candidates for $(z, A, \mathcal{M})$ so that they depend in a Borel-measurable way on $z \in \mathcal{M}$. Here an $\epsilon$-candidate for $(z, A, \mathcal{M})$ is a holomorphic $\operatorname{disc} \phi \in \mathcal{O}(\bar{E}, \mathcal{M})$ such that $\phi(0)=z$ and $\frac{1}{2 \pi} \int_{0}^{2 \pi} 1_{\partial E \backslash \phi^{-1}(A), \partial E}\left(e^{i \theta}\right) d \theta<\mathcal{P}\left[1_{\mathcal{M} \backslash A, \mathcal{M}}\right](z)+\epsilon$.

Using this we can adapt the proof of Theorem 3.8 in order to obtain the desired result.

\section{A mixed cross theorem}

Let $E$ be as usual the open unit disc in $\mathbb{C}$. Let $B$ be a measurable subset of $\partial E$ and $\omega(\cdot, B, E)$ the relative extremal function of $B$ relative to $E$ (with respect to the 
canonical system of approach regions). Then it is well-known (see [40]) that

$$
\omega(z, B, E)=\frac{1}{2 \pi} \int_{0}^{2 \pi} \frac{1-|z|^{2}}{\left|e^{i \theta}-z\right|^{2}} \cdot 1_{\partial E \backslash B, \partial E}\left(e^{i \theta}\right) d \theta .
$$

The following elementary lemma will be very useful.

Lemma 4.1. We keep the above hypotheses and notation.

1) Let $u$ be a subharmonic function defined on $E$ with $u \leq 1$ and let $\alpha \in\left(0, \frac{\pi}{2}\right)$ be such that

$$
\limsup _{z \rightarrow \zeta, z \in \mathcal{A}_{\alpha}(\zeta)} u(z) \leq 0 \quad \text { for a.e. } \zeta \in B
$$

where $\mathcal{A}=\left(\mathcal{A}_{\alpha}(\zeta)\right)$ is the system of angular approach regions defined in $\mathrm{Sub}$ section 2.2. Then $u \leq \omega(\cdot, B, E)$ on $E$.

2) $\omega(\cdot, B, E)$ is also the relative extremal function of $B$ relative to $E$ (with respect to the system of angular approach regions).

3) For all subsets $\mathcal{N} \subset \partial E$ with $\operatorname{mes}(\mathcal{N})=0, \omega(\cdot, B, E)=\omega(\cdot, B \cup \mathcal{N}, E)$.

4) Let $B^{\prime}$ be the set of all density points of $B$. Then

$$
\lim _{z \rightarrow \zeta, z \in \mathcal{A}_{\alpha}(\zeta)} \omega(z, B, E)=0, \quad \zeta \in B^{\prime}, 0<\alpha<\frac{\pi}{2} .
$$

In particular, $B^{\prime}$ is locally pluriregular (with respect to the system of angular approach regions).

5) $\omega(\cdot, B, E)=\widetilde{\omega}_{c}(\cdot, B, E)=\widetilde{\omega}_{a}(\cdot, B, E)$ on $E$, where $\widetilde{\omega}_{c}(\cdot, B, E)$ (respectively $\left.\widetilde{\omega}_{a}(\cdot, B, E)\right)$ is given by Definition 2.3 relative to the system of canonical approach regions (respectively angular approach regions).

Proof. It follows immediately from the explicit formula (4.1). theorem.

The main ingredient in the proof of Theorem $\mathrm{A}$ is the following mixed cross

Theorem 4.2. Let $D$ be a complex manifold and $E$ as usual the open unit disc in $\mathbb{C}$. $D$ (respectively $E$ ) is equipped with the canonical system of approach regions (respectively the system of angular approach regions). Let $A$ be an open subset of $D$ and $B$ a measurable subset of $\partial E$ such that $B$ is locally pluriregular (relative to the system of angular approach regions). For $0 \leq \delta<1$ put $G:=$ $\{w \in E: \omega(w, B, E)<1-\delta\}$. Let $W:=\mathbb{X}(A, B ; D, G), W^{o}:=\mathbb{X}^{o}(A, B ; D, G)$, and $^{6}$

$$
\widehat{W}=\widehat{\mathbb{X}}(A, B ; D, G):=\left\{(z, w) \in D \times G: \omega(z, A, D)+\frac{\omega(w, B, E)}{1-\delta}<1\right\} .
$$

Let $f: W \longrightarrow \mathbb{C}$ be such that

${ }^{6}$ In fact, Theorem 4.10 in [35] says that $\omega(\cdot, B, G)=\frac{\omega(\cdot, B, E)}{1-\delta}$ on $G$, where $\omega(\cdot, B, G)$ is the relative extremal function with respect to the system of angular approach regions induced onto $G$. 
(i) $f \in \mathcal{O}_{s}\left(W^{o}, \mathbb{C}\right)$;

(ii) $f$ is locally bounded on $W,\left.f\right|_{A \times B}$ is a Borel function;

(iii) for all $z \in A$,

$$
\lim _{w \rightarrow \eta, w \in \mathcal{A}_{\alpha}(\eta)} f(z, w)=f(z, \eta), \quad \eta \in B, 0<\alpha<\frac{\pi}{2} .
$$

Then there is a unique function $\hat{f} \in \mathcal{O}(\widehat{W}, \mathbb{C})$ such that $\hat{f}=f$ on $A \times G$. Moreover, $|f|_{W}=|\hat{f}|_{\widehat{W}}$.

The proof of this theorem will occupy the present and the next sections. Our approach here avoid completely the classical method of doubly orthogonal bases of Bergman type. For the proof we need the following "measurable" version of Gonchar's theorem.

Theorem 4.3. Let $D=G:=E$ be equipped with the system of angular approach regions. Let $A$ (respectively $B$ ) be a Borel measurable subset of $\partial D$ (respectively $\partial G)$ such that $A$ and $B$ are locally pluriregular and that $\operatorname{mes}(A), \operatorname{mes}(B)>0$. Put $W:=\mathbb{X}(A, B ; D, G)$ and define $W^{o}, \widehat{W}, \omega(z, w)$ as in Subsection 2.3. Let $f: W \longrightarrow \mathbb{C}$ be such that:

(i) $f$ is locally bounded on $W$ and $f \in \mathcal{O}_{S}\left(W^{o}, \mathbb{C}\right)$;

(ii) $\left.f\right|_{A \times B}$ is a Borel function;

(iii) for all $a \in A$ (respectively $b \in B$ ), $\left.f(a, \cdot)\right|_{G}$ (respectively $\left.\left.f(\cdot, b)\right|_{D}\right)$ admits $\mathcal{A}$-limit ${ }^{7} f(a, b)$ at all $b \in B$ (respectively at all $a \in A$ ).

Then there exists a unique function $\hat{f} \in \mathcal{O}(\widehat{W}, \mathbb{C})$ which admits A-limit $f(\zeta, \eta)$ at all points $(\zeta, \eta) \in W^{o}$.If, moreover, $|f|_{W}<\infty$, then

$$
|\hat{f}(z, w)| \leq|f|_{A \times B}^{1-\omega(z, w)}|f|_{W}^{\omega(z, w)}, \quad(z, w) \in \widehat{W} .
$$

Proof. It follows from Steps 1-3 of Section 6 in [35].

The above theorem is also true in the context of an $N$-fold cross $W(N \geq 2)$. We give here a version of a special 3-fold cross which is needed for the proof of Theorem 4.2.

Theorem 4.4. Let $D=G:=E$ be equipped with the system of angular approach regions. Let $A$ (respectively $B$ ) be a Borel measurable subset of $\partial D$ (respectively $\partial G)$ such that $A$ and $B$ are locally pluriregular and that $\operatorname{mes}(A), \operatorname{mes}(B)>0$.

7 that is, the angular limit 
Define $W, W^{o}, \widehat{W}$ as follows:

$$
\begin{aligned}
W & =\mathbb{X}(A, \partial E, B ; D, E, G) \\
& :=(D \cup A) \times \partial E \times B \bigcup A \times \bar{E} \times B \bigcup A \times \partial E \times(G \cup B), \\
W^{o} & =\mathbb{X}^{o}(A, \partial E, B ; D, E, G):=D \times \partial E \times B \bigcup A \times E \times B \bigcup A \times \partial E \times G, \\
\widehat{W} & =\widehat{\mathbb{X}}(A, \partial E, B ; D, E, G) \\
& :=\{(z, t, w) \in D \times E \times G: \omega(z, A, D)+\omega(w, B, G)<1\} .
\end{aligned}
$$

Let $f: W \longrightarrow \mathbb{C}$ be such that:

(i) $f$ is locally bounded on $W$ and $f \in \mathcal{O}_{S}\left(W^{o}, \mathbb{C}\right)^{8}$;

(ii) $\left.f\right|_{A \times \partial E \times B}$ is a Borel function;

(iii) for all $(a, \lambda) \in A \times \partial E$ (respectively $(a, b) \in A \times B)$ (respectively $(\lambda, b) \in$ $\partial E \times B),\left.f(a, \lambda, \cdot)\right|_{G}$ (respectively $\left.\left.f(a, \cdot, b)\right|_{E}\right)$ (respectively $\left.f(\cdot, \lambda, b)\right|_{D}$ ) admits the angular limit $f(a, \lambda, b)$ at all $b \in B$ (respectively at all $\lambda \in \partial E$ ) (respectively at all $a \in A$ ).

Then there exists a unique function $\hat{f} \in \mathcal{O}(\widehat{W}, \mathbb{C})$ such that

$$
\begin{aligned}
\lim _{\widehat{W} \ni(z, t, w) \rightarrow(\zeta, \tau, \eta), w \in \mathcal{A}_{\alpha}(\eta)} \hat{f}(z, t, w) & =\frac{1}{2 \pi i} \int_{\partial E} \frac{f(\zeta, \lambda, \eta)}{\lambda-\tau} d \lambda, \\
(\zeta, \tau, \eta) & \in D \times E \times B, 0<\alpha<\frac{\pi}{2} .
\end{aligned}
$$

If, moreover, $|f|_{W}<\infty$, then

$$
|\hat{f}(z, t, w)| \leq|f|_{A \times \partial E \times B}^{1-\omega(z, A, D)-\omega(w, B, G)}|f|_{W}^{\omega(z, A, D)+\omega(w, B, G)}, \quad(z, t, w) \in \widehat{W} .
$$

Proof. We refer the reader to Subsections 5.2 and 5.3 in [35].

Let $\hat{\omega}(\cdot, A, D)$ (respectively $\hat{\omega}(\cdot, B, G)$ ) be the conjugate harmonic function of $\omega(\cdot, A, D)$ (respectively $\omega(\cdot, B, G)$ ) such that $\hat{\omega}\left(z_{0}, A, D\right)=0$ (respectively $\hat{\omega}\left(w_{0}, B, G\right)=0$ ) for a certain fixed point $z_{0} \in D$ (respectively $w_{0} \in G$ ). Thus we define the holomorphic functions $g_{1}(z):=\omega(z, A, D)+i \hat{\omega}(z, A, D), g_{2}(w):=$ $\omega(w, B, G)+i \hat{\omega}(w, B, G)$, and

$$
g(z, w):=g_{1}(z)+g_{2}(w), \quad(z, w) \in D \times G .
$$

Each function $e^{-g_{1}}$ (respectively $e^{-g_{2}}$ ) is bounded on $D$ (respectively on $G$ ). Therefore, in virtue of [11, page 439], we may define $e^{-g_{1}(a)}$ (respectively $e^{-g_{2}(b)}$ ) for a.e. $a \in A$ (respectively for a.e. $b \in B$ ) to be the angular limit of $e^{-g_{1}}$ at $a$ (respectively $e^{-g_{2}}$ at $\left.b\right)$.

8 This notation means that for all $(a, \lambda) \in A \times \partial E$ (respectively $(a, b) \in A \times B)$ (respectively $(\lambda, b) \in \partial E \times B)$, the function $\left.f(a, \lambda, \cdot)\right|_{G}$ (respectively $\left.f(a, \cdot, b)\right|_{E}$ ) (respectively $\left.f(\cdot, \lambda, b)\right|_{D}$ ) is holomorphic. 
In virtue of (i), for each positive integer $N$, we define, as in [12,13] (see also [35]), the Gonchar-Carleman operator as follows

$$
\begin{aligned}
K_{N}(z, t, w) & =K_{N}[f](z, t, w) \\
& :=\frac{1}{(2 \pi i)^{2}} \int_{A \times B} e^{-N(g(a, b)-g(z, w))} \frac{f(a, t, b) d a d b}{(a-z)(b-w)}
\end{aligned}
$$

for $(z, t, w) \in D \times \partial E \times G$. Reasoning as in [13] and using (i)-(iii) above, we see that the following limit

$$
K(z, t, w)=K[f](z, t, w):=\lim _{N \rightarrow \infty} K_{N}(z, t, w)
$$

exists for all points in the set $\{(z, t, w): t \in \partial E,(z, w) \in \widehat{\mathbb{X}}(A, B ; D, G)\}$, and its limit is uniform on compact subsets of the latter set.

Observe that for $n=0,1,2, \ldots$, and $N=1,2, \ldots$,

$$
\begin{aligned}
& \int_{\partial E} t^{n} K_{N}(z, t, w) d t \\
& =\frac{1}{(2 \pi i)^{2}} \int_{A \times B}\left(\int_{\partial E} t^{n} f(a, t, b) d t\right) \frac{e^{-N(g(a, b)-g(z, w))} d a d b}{(a-z)(b-w)}=0,
\end{aligned}
$$

where the first equality follows from (4.2), the second one from the equality $\int_{\partial E} t^{n} f(a, t, b) d t=0$ which itself is an immediate consequence of (i). Therefore, we deduce from (4.3) that

$$
\int_{\partial E} t^{n} K(z, t, w) d t=0, \quad(z, w) \in \widehat{\mathbb{X}}(A, B ; D, G), n=0,1,2, \ldots
$$

On the other hand,

$$
\widehat{W}=\{(z, t, w): t \in E,(z, w) \in \widehat{\mathbb{X}}(A, B ; D, G)\} .
$$

Hence, we are able to define the desired extension function

$$
\hat{f}(z, t, w):=\frac{1}{2 \pi i} \int_{\partial E} \frac{K(z, \lambda, w)}{\lambda-t} d \lambda, \quad(z, t, w) \in \widehat{W} .
$$

Recall from Steps 1-3 of Section 6 in [35] that

$\lim _{\widehat{W} \ni(z, w) \rightarrow(\zeta, \eta), w \in \mathcal{A}_{\alpha}(\eta)} K(z, t, w)=f(\zeta, t, \eta), \quad(\zeta, t, \eta) \in D \times \partial E \times B, 0<\alpha<\frac{\pi}{2}$.

Inserting this into the above formula of $\hat{f}$, the desired conclusion of the theorem follows. 
We break the proof of Theorem 4.2 into two cases.

Case 1. $\delta=0$ (that is $G=E$ ).

We follow essentially the arguments presented in Section 4 of [28]. For the sake of clarity and completeness we give here the most basic arguments.

We begin the proof with the following lemma.

Lemma 4.5. We keep the hypothesis of Theorem 4.2. For $j \in\{1,2\}$, let $\phi_{j} \in$ $\mathcal{O}(\bar{E}, D)$ be a holomorphic disc, and let $t_{j} \in E$ such that $\phi_{1}\left(t_{1}\right)=\phi_{2}\left(t_{2}\right)$ and $\frac{1}{2 \pi} \int_{0}^{2 \pi} 1_{D \backslash A, D}\left(\phi_{j}\left(e^{i \theta}\right)\right) d \theta<1$. Then:

1) For $j \in\{1,2\}$, the function $(t, w) \mapsto f(\phi(t), w)$ defined on $\mathbb{X}\left(\phi_{j}^{-1}(A) \cap\right.$ $\partial E, B ; E, G)$ satisfies the hypothesis of Theorem 4.3 , where $\phi_{j}^{-1}(A):=\{t \in$ $\left.\bar{E}: \phi_{j}(t) \in A\right\}$.

2) For $j \in\{1,2\}$, in virtue of Part 1$)$, let $\hat{f}_{j}$ be the unique function in $\mathcal{O}\left(\widehat{\mathbb{X}}\left(\phi_{j}^{-1}(A) \cap \partial E, B ; E, G\right), \mathbb{C}\right)$ given by Theorem 4.3. Then

$$
\hat{f}_{1}\left(t_{1}, w\right)=\hat{f}_{2}\left(t_{2}, w\right)
$$

for all $w \in G$ such that $\left(t_{j}, w\right) \in \widehat{\mathbb{X}}\left(\phi_{j}^{-1}(A) \cap \partial E, B ; E, G\right), j \in\{1,2\}$.

Proof Lemma 4.5. Part 1) follows immediately from the hypothesis. Therefore, it remains to prove Part 2). To do this fix $w_{0} \in G$ such that $\left(t_{j}, w_{0}\right) \in$ $\widehat{\mathbb{X}}\left(\phi_{j}^{-1}(A) \cap E, B ; E, G\right)$ for $j \in\{1,2\}$. We need to show that $\hat{f}_{1}\left(t_{1}, w_{0}\right)=$ $\hat{f}_{2}\left(t_{2}, w_{0}\right)$. Observe that both functions $w \in \mathcal{G} \mapsto \hat{f}_{1}\left(t_{1}, w\right)$ and $w \in \mathcal{G} \mapsto$ $\hat{f}_{2}\left(t_{2}, w\right)$ belong to $\mathcal{O}(\mathcal{G}, \mathbb{C})$, where $\mathcal{G}$ is the connected component which contains $w_{0}$ of the following open set

$$
\left\{w \in G: \omega(w, B, G)<1-\max _{j \in\{1,2\}} \omega\left(t_{j}, \phi_{j}^{-1}(A) \cap \partial E, E\right)\right\} .
$$

Since $\phi_{1}\left(t_{1}\right)=\phi_{2}\left(t_{2}\right)$, it follows from Theorem 4.3 and the hypothesis of Part 2) that

$$
\left(\mathcal{A}-\lim \hat{f}_{1}\right)\left(t_{1}, \eta\right)=f\left(\phi_{1}\left(t_{1}\right), \eta\right)=f\left(\phi_{2}\left(t_{2}\right), \eta\right)=\left(\mathcal{A}-\lim \hat{f}_{2}\right)\left(t_{2}, \eta\right), \quad \eta \in B
$$

Therefore, by Theorem 3.7, $\hat{f}_{1}\left(t_{1}, w\right)=\hat{f}_{2}\left(t_{2}, w\right), w \in \mathcal{G}$. Hence, $\hat{f}_{1}\left(t_{1}, w_{0}\right)=$ $\hat{f}_{2}\left(t_{2}, w_{0}\right)$, which completes the proof of the lemma.

Now we return to the proof of the theorem in Case 1 which is divided into two steps.

Step 1. Construction of the extension function $\hat{f}$ on $\widehat{W}$ and its uniqueness. 
Proof of Step 1. We define $\hat{f}$ as follows: Let $\mathcal{W}$ be the set of all pairs $(z, w) \in D \times$ $G$ with the property that there are a holomorphic disc $\phi \in \mathcal{O}(\bar{E}, D)$ and $t \in E$ such that $\phi(t)=z$ and $(t, w) \in \widehat{\mathbb{X}}\left(\phi^{-1}(A) \cap \partial E, B ; E, G\right)$. By Part 1) of Lemma 4.5 and Theorem 4.3 , let $\hat{f}_{\phi}$ be the unique function in $\mathcal{O}\left(\widehat{\mathbb{X}}\left(\phi^{-1}(A) \cap \partial E, B ; E, G\right), \mathbb{C}\right)$ such that

$$
\left(\mathcal{A}-\lim \hat{f}_{\phi}\right)(t, w)=f(\phi(t), w), \quad(t, w) \in \mathbb{X}^{\mathrm{o}}\left(\phi^{-1}(A) \cap \partial E, B ; E, G\right) .
$$

Then the desired extension function $\hat{f}$ is given by

$$
\hat{f}(z, w):=\hat{f}_{\phi}(t, w) \text {. }
$$

In virtue of Part 2) of Lemma 4.5, $\hat{f}$ is well-defined on $\mathcal{W}$. We next prove that

$$
\mathcal{W}=\widehat{W}
$$

Taking (4.6) for granted, then $\hat{f}$ is well-defined on $\widehat{W}$.

Now we return to (4.6). To prove the inclusion $\mathcal{W} \subset \widehat{W}$, let $(z, w) \in \mathcal{W}$. By the above definition of $\mathcal{W}$, one may find a holomorphic disc $\phi \in \mathcal{O}(\bar{E}, D)$, a point $t \in E$ such that $\phi(t)=z$ and $(t, w) \in \widehat{\mathbb{X}}\left(\phi^{-1}(A) \cap \partial E, B ; E, G\right)$. Since $\omega(\phi(t), A, D) \leq \omega\left(t, \phi^{-1}(A) \cap \partial E, E\right)$, it follows that

$$
\omega(z, A, D)+\omega(w, B, G) \leq \omega\left(t, \phi^{-1}(A) \cap \partial E, E\right)+\omega(w, B, G)<1 .
$$

Hence $(z, w) \in \widehat{W}$. This proves the above mentioned inclusion.

To finish the proof of (4.6), it suffices to show that $\widehat{W} \subset \mathcal{W}$. To do this, let $(z, w) \in \widehat{W}$ and fix any $\epsilon>0$ such that

$$
\epsilon<1-\omega(z, A, D)-\omega(w, B, G) .
$$

Applying Theorem 3.1 and Proposition 3.4, there is a holomorphic disc $\phi \in \mathcal{O}(\bar{E}, D)$ such that $\phi(0)=z$ and

$$
\frac{1}{2 \pi} \int_{0}^{2 \pi} 1_{D \backslash A, D}\left(\phi\left(e^{i \theta}\right)\right) d \theta<\omega(z, A, D)+\epsilon .
$$

Observe that

$$
\begin{aligned}
\omega\left(0, \phi^{-1}(A) \cap \partial E, E\right)+\omega(w, B, G) & =\frac{1}{2 \pi} \int_{0}^{2 \pi} 1_{D \backslash A, D}\left(\phi\left(e^{i \theta}\right)\right) d \theta+\omega(w, B, G) \\
& <\omega(z, A, D)+\omega(w, B, G)+\epsilon<1,
\end{aligned}
$$

where the equality follows from (4.1), the first inequality holds by (4.8), and the last one by (4.7). Hence, $(0, w) \in \widehat{\mathbb{X}}\left(\phi^{-1}(A) \cap \partial E, B ; E, G\right)$, which implies that 
$(z, w) \in \mathcal{W}$. This completes the proof of (4.6). Hence, the construction of $\hat{f}$ on $\widehat{W}$ has been completed.

Next we show that $\hat{f}=f$ on $A \times G$. To this end let $\left(z_{0}, w_{0}\right)$ be an arbitrary point of $A \times G$. Choose the holomorphic disc $\phi \in \mathcal{O}(\bar{E}, D)$ given by $\phi(t):=z_{0}$, $t \in \bar{E}$. Then by formula (4.5),

$$
\hat{f}\left(z_{0}, w_{0}\right)=\hat{f}_{\phi}\left(0, w_{0}\right)=f\left(\phi(0), w_{0}\right)=f\left(z_{0}, w_{0}\right) .
$$

If $g \in \mathcal{O}(\widehat{W}, \mathbb{C})$ satisfies $g=f$ on $A \times G$, then we deduce from (4.4)-(4.5) that $g=\hat{f}$. This proves the uniqueness of $\hat{f}$.

Finally, we conclude the proof of Case 1 by the following

Step 2. Proof of the fact that $\hat{f} \in \mathcal{O}(\widehat{W}, \mathbb{C})$.

Proof of Step 2. Fix an arbitrary point $\left(z_{0}, w_{0}\right) \in \widehat{W}$ and let $\epsilon>0$ be so small such that

$$
2 \epsilon<1-\omega\left(z_{0}, A, D\right)-\omega\left(w_{0}, B, G\right) .
$$

Since $\omega(\cdot, B, G) \in \mathcal{P} \mathcal{S H}(G)$, one may find an open neighborhood $V$ of $w_{0}$ such that

$$
\omega(w, B, D)<\omega\left(w_{0}, B, G\right)+\epsilon, \quad w \in V .
$$

Let $n$ be the dimension of $D$ at the point $z_{0}$. Applying Lemma 3.2 and Proposition 3.4, we obtain an open set $T$ in $\mathbb{C}$, an open neighborhood $U$ of $z_{0}$, and a family of holomorphic discs $\left(\phi_{z}\right)_{z \in U} \subset \mathcal{O}(\bar{E}, D)$ with the following properties:

$$
\begin{aligned}
\text { the mapping }(z, t) & \in U \times E \mapsto \phi_{z}(t) \text { is holomorphic; } \\
\phi_{z}(0) & =z, \quad z \in U ; \\
\phi_{z}(t) & \in A, \quad t \in T \cap \bar{E}, z \in U ; \\
\frac{1}{2 \pi} \int_{0}^{2 \pi} 1_{\partial E \backslash T, \partial E}\left(e^{i \theta}\right) d \theta & <\omega\left(z_{0}, A, D\right)+\epsilon .
\end{aligned}
$$

By shrinking $U$ (if necessary), we may assume without loss of generality that in a chart, $z_{0}=0 \in \mathbb{C}^{n}$ and

$$
U=\left\{z=\left(z^{1}, \ldots, z^{n}\right)=\left(z^{\prime}, z^{n}\right) \in \mathbb{C}^{n}: z^{\prime} \in S,\left|z^{n}\right|<2\right\},
$$

where $S \subset \mathbb{C}^{n-1}$ is an open set.

Consider the 3 -fold cross (compared with the notation in Theorem 4.4)

$$
\begin{aligned}
& \mathbb{X}(T \cap \partial E, U, B ; E, U, G):=(E \cup(T \cap \partial E)) \times U \times B \\
& \bigcup(T \cap \partial E) \times U \times B \bigcup(T \cap \partial E) \times U \times(G \cup B),
\end{aligned}
$$


and the function $g: \mathbb{X}(T \cap \partial E, U, B ; E, U, G) \longrightarrow \mathbb{C}$ given by

$$
g(t, z, w):=f\left(\phi_{z}(t), w\right), \quad(t, z, w) \in \mathbb{X}(T \cap \partial E, U, B ; E, U, G) .
$$

We make the following observations:

Let $t \in T \cap \partial E$. Then, in virtue of (4.13) we have $\phi_{z}(t) \in A$ for $z \in U$. Consequently, in virtue of (4.11), (4.16) and the hypothesis $f \in \mathcal{O}_{s}\left(W^{\mathrm{o}}, \mathbb{C}\right)$, we conclude that $\left.g(t, z, \cdot)\right|_{G} \in \mathcal{O}(G, \mathbb{C})$ (respectively $\left.g(t, \cdot, w)\right|_{U} \in \mathcal{O}(U, \mathbb{C})$ ) for any $z \in U$ (respectively $w \in B$ ). Analogously, for any $z \in U, w \in B$, we can show that $\left.g(\cdot, z, w)\right|_{E} \in \mathcal{O}(E, \mathbb{C})$.

In summary, we have shown that $g$ is separately holomorphic. In addition, it follows from hypothesis (ii) and (4.11)-(4.13) that $g$ is locally bounded and $\left.g\right|_{(T \cap \partial E) \times U \times B}$ is a Borel function.

For $z^{\prime} \in S$ write $E_{z^{\prime}}:=\left\{z=\left(z^{\prime}, z_{n}\right) \in \mathbb{C}^{n}:\left|z_{n}\right|<1\right\}$. Then by (4.15), $\bigcup_{z^{\prime} \in S} E_{z^{\prime}} \subset U$. Consequently, for all $z^{\prime} \in S$, using hypothesis (iii) we are able to apply Theorem 4.4 to $g$ in order to obtain a unique function $\hat{g} \in \mathcal{O}(\widehat{\mathbb{X}}(T \cap$ $\left.\left.\partial E, \partial E_{z^{\prime}}, B ; E, E_{z^{\prime}}, G\right), \mathbb{C}\right)^{9}$ such that

$$
\begin{aligned}
\lim _{(t, z, w) \rightarrow(\tau, \zeta, \eta), w \in \mathcal{A}_{\alpha}(\eta)} \hat{g}(t, z, w) & =\frac{1}{2 \pi i} \int_{\partial E_{z^{\prime}}} \frac{g\left(\tau, \zeta^{\prime}, \lambda, \eta\right)}{\lambda-\zeta_{n}} d \lambda, \\
(\tau, \zeta, \eta) & \in E \times E_{z^{\prime}} \times B, z^{\prime} \in S, 0<\alpha<\frac{\pi}{2} .
\end{aligned}
$$

Using (4.11) and (4.15)-(4.16) and the Cauchy's formula, we see that the right hand side is equal to $g(\tau, \zeta, \eta)$. Hence, we have shown that

$$
\begin{aligned}
& \lim _{(t, z, w) \rightarrow(\tau, \zeta, \eta), w \in \mathcal{A}_{\alpha}(\eta)} \hat{g}(t, z, w)=g(\tau, \zeta, \eta), \\
& (\tau, \zeta, \eta) \in E \times E_{z^{\prime}} \times B, z^{\prime} \in S, 0<\alpha<\frac{\pi}{2} .
\end{aligned}
$$

Observe that

$$
\begin{aligned}
& \widehat{\mathbb{X}}\left(T \cap \partial E, \partial E_{z^{\prime}}, B ; E, E_{z^{\prime}}, G\right) \\
& =\left\{(t, z, w) \in E \times E_{z^{\prime}} \times G: \omega(t, T \cap \partial E, E)+\omega(w, B, G)<1\right\} .
\end{aligned}
$$

On the other hand, for any $w \in V$,

$$
\begin{aligned}
\omega(0, T \cap \partial E, E)+\omega(w, B, G) & \leq \frac{1}{2 \pi} \int_{0}^{2 \pi} 1_{\partial E \backslash T, \partial E}\left(e^{i \theta}\right) d \theta+\omega\left(w_{0}, B, G\right)+\epsilon \\
& <\omega\left(z_{0}, A, D\right)+\omega\left(w_{0}, B, G\right)+2 \epsilon<1,
\end{aligned}
$$

${ }^{9}$ In fact, we identify $E_{z^{\prime}}$ with $E$ in an obvious way. 
where the first inequality follows from (4.1) and (4.10), the second one from (4.14), and the last one from (4.9). Consequently,

$$
(0, z, w) \in \widehat{\mathbb{X}}\left(T \cap \partial E, \partial E_{z^{\prime}}, B ; E, E_{z^{\prime}}, G\right), \quad(z, w) \in E_{z^{\prime}} \times V, z^{\prime} \in S .
$$

It follows from (4.5), (4.12), (4.13) and (4.18) that, for $z^{\prime} \in S$ and $z \in E_{z^{\prime}}, \hat{f}_{\phi_{z}}$ is well-defined and holomorphic on $\widehat{\mathbb{X}}(T \cap \partial E, B ; E, G)$, and

$$
\hat{f}(z, w)=\hat{f}_{\phi_{z}}(0, w), \quad w \in V
$$

On the other hand, it follows from (4.4), (4.16) and (4.17) that

$$
\begin{aligned}
& \lim _{(t, w) \rightarrow(\tau, \eta), w \in \mathcal{A}_{\alpha}(\eta)} \hat{f}_{\phi_{z}}(t, w)=\lim _{(t, w) \rightarrow(\tau, \eta), w \in \mathcal{A}_{\alpha}(\eta)} \hat{g}(t, z, w), \\
& (\tau, \eta) \in E \times B, z \in E_{z^{\prime}}, z^{\prime} \in S, 0<\alpha<\frac{\pi}{2} .
\end{aligned}
$$

Since, for fixed $z \in E_{z^{\prime}}$, the restricted functions $(t, w) \mapsto \hat{g}(t, z, w)$ and $\hat{f}_{\phi_{z}}$ are holomorphic on $\widehat{\mathbb{X}}(T \cap \partial E, B ; E, G)$, we deduce from the latter equality and the uniqueness of Theorem 4.3 that

$$
\hat{g}(t, z, w)=\hat{f}_{\phi_{z}}(t, w), \quad(t, w) \in \widehat{\mathbb{X}}(T \cap \partial E, B ; E, G), z \in E_{z^{\prime}}, z^{\prime} \in S .
$$

In particular, using (4.5), (4.19) and (4.20),

$$
\hat{g}(0, z, w)=\hat{f}_{\phi_{z}}(0, w)=\hat{f}(z, w), \quad(z, w) \in E_{z^{\prime}} \times V, z^{\prime} \in S .
$$

Since we know from (4.19) that $\hat{g}$ is holomorphic in the variables $z^{n}$ and $w$ on a neighborhood of $\left(0, z_{0}, w_{0}\right)$, it follows that $\hat{f}$ is holomorphic in the variables $z^{n}$ and $w$ on a neighborhood of $\left(z_{0}, w_{0}\right)$. Exchanging the role of $z^{n}$ and any other variable $z^{j}, j=1, \ldots, n-1$, we see that $\hat{f}$ is separetely holomorphic on a neighborhood of $\left(z_{0}, w_{0}\right)$. In addition, $\hat{f}$ is locally bounded. Consequently, we conclude, by the classical Hartogs extension theorem, that $\hat{f}$ is holomorphic on a neighborhood of $\left(z_{0}, w_{0}\right)$. Since $\left(z_{0}, w_{0}\right) \in \widehat{W}$ is arbitrary, it follows that $\hat{f} \in \mathcal{O}(\widehat{W}, \mathbb{C})$.

Combining Steps 1-2, Case 1 follows.

\section{Completion of the proof of Theorem 4.2}

In this section we introduce the new technique of conformal mappings. This technique will allow us to pass from Case 1 to the general case. We recall a notion from Definition 4.8 in [35] which will be relevant for our further study. 
Definition 5.1. Let $\mathcal{A}$ be the system of angular approach regions for $E$, let $\Omega$ be an open subset of the unit disc $E$ and $\zeta$ a point in $\partial E$. Then the point $\zeta$ is said to be an end-point of $\Omega$ if, for every $0<\alpha<\frac{\pi}{2}$, there is an open neighborhood $U=U_{\alpha}$ of $\zeta$ such that $U \cap \mathcal{A}_{\alpha}(\zeta) \subset \Omega$. The set of all end-points of $\Omega$ is denoted by $\operatorname{End}(\Omega)$.

The main idea of the technique of conformal mappings is described below.

Proposition 5.2. Let $B$ be a measurable subset of $\partial E$ with $\operatorname{mes}(B)>0$. For $0 \leq$ $\delta<1$ put $G:=\{w \in E: \omega(w, B, E)<1-\delta\}$. Let $\Omega$ be an arbitrary connected component of $G$. Then

1) $\operatorname{End}(\Omega)$ is a measurable subset of $\partial E$ and $\operatorname{mes}(\operatorname{End}(\Omega))>0$. Moreover, $\Omega$ is a simply connected domain.

In virtue of Part 1) and the Riemann mapping theorem, let $\Phi$ be a conformal mapping of $\Omega$ onto $E$.

2) For every $\zeta \in \operatorname{End}(\Omega)$, there is $\eta \in \partial E$ such that

$$
\lim _{z \rightarrow \zeta, \Omega \Omega \cap \mathcal{A}_{\alpha}(\zeta)} \Phi(z)=\eta, \quad 0<\alpha<\frac{\pi}{2} .
$$

$\eta$ is called the limit of $\Phi$ at the end-point $\zeta$ and it is denoted by $\Phi(\zeta)$. Moreover, $\left.\Phi\right|_{\operatorname{End}(\Omega)}$ is one-to-one.

3) Let $f$ be a bounded holomorphic function on $\Omega, \zeta \in \operatorname{End}(\Omega)$ and $\lambda \in \mathbb{C}$ such that $\lim _{z \rightarrow \zeta, z \in \Omega \cap \mathcal{A}_{\alpha}(\zeta)} f(z)=\lambda$ for some $0<\alpha<\frac{\pi}{2}$. Then $f \circ \Phi^{-1} \in$ $\mathcal{O}(E, \mathbb{C})$ admits the angular limit $\lambda$ at $\Phi(\zeta)$.

4) Let $\Delta$ be a subset of $\operatorname{End}(\Omega)$ such that $\operatorname{mes}(\Delta)=\operatorname{mes}(\operatorname{End}(\Omega))$. Put $\Phi(\Delta):=$ $\{\Phi(\zeta), \zeta \in \Delta\}$, where $\Phi(\zeta)$ is given by Part 2$)$. Then $\Phi(\Delta)$ is a measurable subset of of $\partial E$ with mes $(\Phi(\Delta))>0$. and

$$
\omega(\Phi(z), \Phi(\Delta), E)=\frac{\omega(z, B, E)}{1-\delta}, \quad z \in \Omega .
$$

Proof. The first assertions of Part 1) follows from Theorem 4.9 in [35]. To show that $\Omega$ is simply connected, take an arbitrary Jordan domain $D$ such that $\partial D \subset \Omega$. We need to prove that $D \subset \Omega$. Observe that $D \subset E$ and $\omega(z, B, E)<1-\delta$ for all $z \in \partial D \subset \Omega \subset G$. By the Maximum Principle, we deduce that $\omega(z, B, E)<1-\delta$ for all $z \in D$. Hence, $D \subset G$, which, in turn, implies that $D \subset \Omega$. This completes Part 1).

Part 2) follows from the "end-point" version of Theorem 4.4.13 in [40] (that is, we replace the hypothesis "accessible point" therein by end-point).

Applying the classical Lindelöf's theorem to $f \circ \Phi^{-1} \in \mathcal{O}(E, \mathbb{C})$, Part 3) follows.

It remains to prove Part 4). A straightforward argument shows that $\Phi(\Delta)$ is a measurable subset of $\partial E$. Next, we show that

$$
\omega(\Phi(z), \Phi(\Delta), E) \leq \frac{\omega(z, B, E)}{1-\delta}, \quad z \in \Omega .
$$


To do this pick any $u \in \mathcal{P S H}(E)$ such that $u \leq 1$ and

$$
\limsup _{w \rightarrow \eta} u(w) \leq 0, \quad \eta \in \Phi(\Delta) .
$$

Consequently, Part 2) gives that

$$
\limsup _{z \rightarrow \zeta, z \in \Omega \cap \mathcal{A}_{\alpha}(\zeta)} u \circ \Phi(z)=0, \quad \zeta \in \Delta, 0<\alpha<\frac{\pi}{2} .
$$

Next, consider the following function

$$
\tilde{u}(z):= \begin{cases}\max \{(1-\delta) \cdot(u \circ \Phi)(z), \omega(z, B, E)\}, & z \in \Omega, \\ \omega(z, B, E), & z \in E \backslash \Omega .\end{cases}
$$

Then it can be checked that $\tilde{u}$ is subharmonic and $\tilde{u} \leq 1$ in $E$. In addition, for every density point $\zeta$ of $B$ such that $\zeta \notin \operatorname{End}(\Omega)$, we know from Theorem 4.9 in [35] that there is a connected component $\Omega_{\zeta}$ of $G$ other than $\Omega$ such that $\zeta \in \operatorname{End}\left(\Omega_{\zeta}\right)$. Consequently, Part 4) of Lemma 4.1 gives, for such a point $\zeta$, that

$$
\limsup _{z \rightarrow \zeta, z \in \mathcal{A}_{\alpha}(\zeta)} \tilde{u}(z)=\limsup _{z \rightarrow \zeta, z \in \mathcal{A}_{\alpha}(\zeta)} \omega(z, B, E)=0, \quad 0<\alpha<\frac{\pi}{2} .
$$

This, combined with (5.2), implies that

$$
\limsup _{z \rightarrow \zeta, z \in \mathcal{A}_{\alpha}(\zeta)} \tilde{u}(z)=0, \quad 0<\alpha<\frac{\pi}{2}, \text { for a.e. } \zeta \in B .
$$

Consequently, applying Part 1) of Lemma 4.1 yields that $\tilde{u} \leq \omega(\cdot, B, E)$ on $E$. Hence, by (5.3), $(u \circ \Phi)(z) \leq \frac{\omega(z, B, E)}{1-\delta}, z \in \Omega$, which completes the proof of (5.1). In particular, we obtain that mes $(\Phi(\Delta))>0$.

To prove the opposite inequality of (5.1), let $u$ be an arbitrary function in $\mathcal{P S H}(E)$ such that $u \leq 1$ and

$$
\limsup _{z \rightarrow \zeta} u(z) \leq 0, \quad \zeta \in B .
$$

Applying Part 3) to the function $f(z):=z$, we obtain that

$$
\limsup _{w \rightarrow \eta, w \in \mathcal{A}_{\alpha}(\eta)} \frac{\left(u \circ \Phi^{-1}\right)(w)}{1-\delta} \leq 0, \quad \eta \in \Phi(\Delta), 0<\alpha<\frac{\pi}{2} .
$$

On the other hand, since $u \leq \omega(\cdot, B, E)$ on $E$, one gets that $\frac{\left(u \circ \Phi^{-1}\right)(w)}{1-\delta} \leq 1$, $w \in E$. Therefore, applying Part 1 ) of Lemma 4.1 yields that

$$
\frac{\left(u \circ \Phi^{-1}\right)(w)}{1-\delta} \leq \omega(w, \Phi(\Delta), E), \quad w \in E,
$$

which, in turn, implies the converse inequality of (5.1). Hence, the proof of Part 4) is complete. 
Now we are in the position to complete the proof of Theorem 4.2:

Case 2. $0<\delta<1$.

Let $\left(G_{k}\right)_{k \in K}$ be the family of all connected components of $G$, where $K$ is an (at most) countable index set. By Proposition 5.2, we may fix a conformal mapping $\Phi_{k}$ from $G_{k}$ onto $E$ for every $k \in K$. Put

$$
\begin{aligned}
B_{k} & :=\left[\Phi_{k}\left(\operatorname{End}\left(G_{k}\right) \cap B\right)\right]^{\prime}, W_{k}:=\mathbb{X}\left(A, B_{k}^{\prime} ; D, E\right), \\
W_{k}^{\mathrm{o}} & :=\mathbb{X}^{\mathrm{o}}\left(A, B_{k}^{\prime} ; D, E\right), \widehat{W}_{k}:=\widehat{\mathbb{X}}\left(A, B_{k}^{\prime} ; D, E\right), \quad k \in K .
\end{aligned}
$$

where $[T]^{\prime}$ (or simply $T^{\prime}$ ) for $T \subset \partial E$ is, following the notation of Lemma 4.1, the set of all density points of $T$.

Recall from the hypotheses of Theorem 4.2 that for every fixed $z \in A$, the holomorphic function $\left.f(z, \cdot)\right|_{G}$ is bounded and that for every $\eta \in B$,

$$
\lim _{w \rightarrow \eta, w \in \Omega \cap \mathcal{A}_{\alpha}(\eta)} f(z, w)=f(z, \eta), \quad 0<\alpha<\frac{\pi}{2} .
$$

Consequently, Part 3) of Proposition 5.2, applied to $\left.f(z, \cdot)\right|_{G_{k}}$ with $k \in K$, implies that for every fixed $z \in D, f\left(z, \Phi_{k}^{-1}(\cdot)\right) \in \mathcal{O}(E, \mathbb{C})$ admits the angular limit $f(z, \eta)$ at $\Phi_{k}(\eta)$ for all $\eta \in B \cap \operatorname{End}\left(G_{k}\right)$. By Part 1$)$ of that proposition, we know that mes $\left(B \cap \operatorname{End}\left(G_{k}\right)\right)>0$. This discussion and the hypothesis allow us to apply the result of Case 1 to the function $g_{k}: W_{k} \longrightarrow \mathbb{C}$ defined by

$$
g_{k}(z, w):= \begin{cases}f\left(z, \Phi_{k}^{-1}(w)\right), & (z, w) \in D \times G_{k}, \\ f\left(z, \Phi_{k}^{-1}(w)\right) & (z, w) \in D \times B_{k}^{\prime},\end{cases}
$$

where in the second line we have used the definition of $\left.\Phi_{k}\right|_{\operatorname{End}\left(G_{k}\right)}$ and its one-toone property proved by Part 2) of Proposition 5.2.

Consequently, we obtain an extension function $\hat{g}_{k} \in \mathcal{O}\left(\widehat{W}_{k}, \mathbb{C}\right)$ such that

$$
\hat{g}_{k}(z, w)=g_{k}(z, w), \quad(z, w) \in A \times E .
$$

Put

$$
\widehat{\mathcal{W}}_{k}:=\left\{\left(z, \Phi_{k}^{-1}(w)\right),(z, w) \in \widehat{W}_{k}\right\}, \quad k \in K .
$$

Observe that the open sets $\left(\widehat{\mathcal{W}}_{k}\right)_{k \in K}$ are pairwise disjoint. Moreover, by (5.4),

$$
\begin{aligned}
& \bigcup_{k \in K} \widehat{\mathcal{W}}_{k}=\left\{(z, w) \in D \times E: w \in G_{k}\right. \text { and } \\
&\left.\omega(z, A, D)+\omega\left(\Phi_{k}(w), \Phi_{k}\left(\operatorname{End}\left(G_{k}\right)\right), E\right)<1 \text { for some } k \in K\right\} \\
&=\left\{(z, w) \in D \times E: w \in G_{k} \text { and } \omega(z, A, D)+\frac{\omega(w, B, E)}{1-\delta}<1 \text { for some } k \in K\right\} \\
&= \widehat{W},
\end{aligned}
$$


where the second equality follows from Part 4) of Proposition 5.2. Therefore, we can define the desired extension function $\hat{f} \in \mathcal{O}(\widehat{W}, \mathbb{C})$ by the formula

$$
\hat{f}(z, w):=\hat{g}_{k}\left(z, \Phi_{k}(w)\right), \quad(z, w) \in \widehat{\mathcal{W}}_{k}, k \in K .
$$

This, combined with (5.4)-(5.6), implies that $\hat{f}=f$ on $A \times G$. The uniqueness of $\hat{f}$ follows from that of $\hat{g}_{k}, k \in K$. Hence, the proof of the theorem is complete.

\section{A local version of Theorem A}

The main purpose of the section is to prove the following result.

Theorem 6.1. Let $D \subset \mathbb{C}^{n}, G \subset \mathbb{C}^{m}$ be bounded open sets. $D$ (respectively $G)$ is equipped with a system of approach regions $\left(\mathcal{A}_{\alpha}(\zeta)\right)_{\zeta \in \bar{D}, \alpha \in I_{\zeta}}$ (respectively $\left(\mathcal{A}_{\alpha}(\eta)\right)_{\eta \in \bar{G}, \alpha \in I_{\eta}}$ ). Let $A$ (respectively $B$ ) be a subset of $\bar{D}$ (respectively $\bar{G}$ ) such that $A$ and $B$ are locally pluriregular. Put

$$
\begin{aligned}
W & :=\mathbb{X}(A, B ; D, G), & \bar{W} & :=\mathbb{X}(\bar{A}, \bar{B} ; D, G), \\
\bar{W}^{o} & :=\mathbb{X}^{o}(\bar{A}, \bar{B} ; D, G), & \widehat{W} & :=\widehat{\mathbb{X}}(A, B ; D, G) .
\end{aligned}
$$

Then, for every bounded function $f: \bar{W} \longrightarrow \mathbb{C}$ such that $f \in \mathcal{C}_{S}(\bar{W}, \mathbb{C}) \cap$ $\mathcal{O}_{s}\left(\bar{W}^{O}, \mathbb{C}\right)$ and that $\left.f\right|_{\bar{A} \times \bar{B}}$ is continuous at all points of $(\bar{A} \cap \partial D) \times(\bar{B} \cap \partial G)$, there exists a unique bounded function $\hat{f} \in \mathcal{O}(\widehat{W}, \mathbb{C})$ which admits $\mathcal{A}$-limit $f(\zeta, \eta)$ at all points $(\zeta, \eta) \in W$. Moreover,

$$
|\hat{f}(z, w)| \leq|f|_{A \times B}^{1-\omega(z, w)}|f|_{W}^{\omega(z, w)}, \quad(z, w) \in \widehat{W} .
$$

The core of our unified approach will be presented in the proof below. Our idea is to use Theorem 3.8 in order to reduce Theorem 6.1 to the case of bidisk, that is, the case of Theorem 4.3. This reduction is based on Theorem 4.2 and on the technique of level sets.

Proof. It is divided into four steps.

Step 1. Construction of the desired function $\hat{f} \in \mathcal{O}(\widehat{W}, \mathbb{C})$ and proof of the estimate $|\hat{f}|_{\widehat{W}} \leq|f|_{\bar{W}}$.

Proof of Step 1. We define $\hat{f}$ at an arbitrary point $(z, w) \in \widehat{W}$ as follows: Let $\epsilon>0$ be such that

$$
\omega(z, A, D)+\omega(w, B, G)+2 \epsilon<1 .
$$

By Theorem 3.8 and Definition 3.9, there is an $\epsilon$-candidate $(\phi, \Gamma)$ (respectively $(\psi, \Delta))$ for $(z, A, D)$ (respectively $(w, B, G))$. Moreover, using the hypotheses, we see that the function $f_{\phi, \psi}$, defined by

$$
f_{\phi, \psi}(t, \tau):=f(\phi(t), \psi(\tau)), \quad(t, \tau) \in \mathbb{X}(\Gamma, \Delta ; E, E),
$$


satisfies the hypotheses of Theorem 4.3. By this theorem, let $\hat{f}_{\phi, \psi}$ be the unique function in $\widehat{\mathbb{X}}(\Gamma, \Delta ; E, E)$ such that

$$
\left(\mathcal{A}-\lim \hat{f}_{\phi, \psi}\right)(t, \tau)=f_{\phi, \psi}(t, \tau), \quad(t, \tau) \in \mathbb{X}^{\mathrm{o}}(\Gamma, \Delta ; E, E) .
$$

In virtue of (6.2) and Theorem 3.8 and Lemma 3.3, $(0,0) \in \widehat{\mathbb{X}}(\Gamma, \Delta ; E, E)$. Then we can define the value of the desired extension function $\hat{f}$ at $(z, w)$ as follows

$$
\hat{f}(z, w):=\hat{f}_{\phi, \psi}(0,0)
$$

The remaining part of this step is devoted to showing that $\hat{f}$ is well-defined and holomorphic on $\widehat{W}$.

To this end we fix an arbitrary point $w_{0} \in G$, a number $\epsilon_{0}: 0<\epsilon_{0}<1-$ $\omega\left(w_{0}, B, G\right)$, and an arbitrary $\epsilon_{0}$-candidate $\left(\psi_{0}, \Delta_{0}\right)$ for $\left(w_{0}, B, G\right)$.

Let

$$
\widehat{W}_{0}:=\left\{(z, \tau) \in D \times E: \omega(z, A, D)+\omega\left(\tau, \Delta_{0}, E\right)<1\right\}
$$

Inspired by formula (6.5) we define a function $\hat{f}_{0}: \widehat{W}_{0} \longrightarrow \mathbb{C}$ as follows

$$
\hat{f}_{0}(z, \tau):=\hat{f}_{\phi, \psi_{0}}(0, \tau)
$$

Here we have used an $\epsilon$-candidate $(\phi, \Gamma)$ for $(z, A, D)$, where $\epsilon$ is arbitrarily chosen so that $0<\epsilon<1-\omega(z, A, D)-\omega\left(\tau, \Delta_{0}, E\right)$.

Using (6.3)-(6.4) and (6.7) and arguing as in Part 2) of Lemma 4.5, one can show that $\hat{f}_{0}$ is well-defined on $\widehat{W}_{0}$.

For all $0<\delta<1$ let

$$
A_{\delta}:=\{z \in D: \omega(z, A, D)<\delta\} \text { and } E_{\delta}:=\left\{w \in E: \omega\left(w, \Delta_{0}, E\right)<1-\delta\right\} .
$$

Then by the construction in (6.7), we remark that $\hat{f}_{0}(z, \cdot)$ is holomorphic on $E_{\delta}$ for every fixed $z \in A_{\delta}$. We are able to define a new function $\tilde{f}_{\delta}$ on $\mathbb{X}\left(A_{\delta}, B ; D, E_{\delta}\right)$ as follows

$$
\tilde{f}_{\delta}(z, \tau):= \begin{cases}\hat{f}_{0}(z, \tau) & (z, \tau) \in A_{\delta} \times E_{\delta} \\ f\left(z, \psi_{0}(\tau)\right) & (z, \tau) \in D \times \Delta_{0} .\end{cases}
$$

Using the hypotheses on $f$ and the previous remark, we see that $\tilde{f}_{\delta} \in \mathcal{O}_{s}\left(\mathbb{X}^{\mathrm{o}}\left(A_{\delta}, B\right.\right.$; $\left.\left.D, E_{\delta}\right), \mathbb{C}\right)$.

Observe that $A_{\delta}$ is an open set in $D$. Consequently, $\tilde{f}_{\delta}$ satisfies the hypotheses of Theorem 4.2. Applying this theorem yields a unique function $\hat{f}_{\delta} \in$ $\mathcal{O}\left(\widehat{\mathbb{X}}\left(A_{\delta}, B ; D, E_{\delta}\right), \mathbb{C}\right)$ such that

$$
\hat{f}_{\delta}(z, w)=\tilde{f}_{\delta}(z, w), \quad(z, w) \in A_{\delta} \times E_{\delta}
$$


This, combined with (6.9), implies that $\hat{f}_{0}$ is holomorphic on $A_{\delta} \times G_{\delta}$. On the other hand, it follows from (6.6) and (6.8) that

$$
\widehat{W}_{0}=\widehat{\mathbb{X}}\left(A, \Delta_{0} ; D, E\right)=\bigcup_{0<\delta<1} A_{\delta} \times G_{\delta}
$$

Hence, $\hat{f}_{0} \in \mathcal{O}\left(\widehat{W}_{0}, \mathbb{C}\right)$.

In summary, we have shown that $\hat{f}_{0}$, given by (6.7), is well-defined and holomorphic on $\widehat{W}_{0}$.

Now we are able to prove that $\hat{f}$, given by (6.5), is well-defined. To this end we fix an arbitrary point $\left(z_{0}, w_{0}\right) \in \widehat{W}$, an $\epsilon_{0}: 0<\epsilon_{0}<1-\omega\left(z_{0}, D, G\right)$, and two arbitrary $\epsilon_{0}$-candidates $\left(\psi_{1}, \Delta_{1}\right)$ and $\left(\psi_{2}, \Delta_{2}\right)$ for $\left(w_{0}, B, G\right)$. Let

$$
\widehat{W}_{j}:=\left\{(z, \tau) \in D \times E: \omega(z, A, D)+\omega\left(\tau, \Delta_{j}, E\right)<1\right\}, \quad j \in\{1,2\} .
$$

Using formula (6.7) define, for $j \in\{1,2\}$, a function $\hat{f}_{j}: \widehat{W}_{j} \longrightarrow \mathbb{C}$ as follows

$$
\hat{f}_{j}(z, \tau):=\hat{f}_{\phi, \psi_{j}}(0, \tau) .
$$

Here we have used any $\epsilon$-candidate $(\phi, \Gamma)$ for $(z, A, D)$ with a suitable $\epsilon>0$. Let $\tau_{j} \in E$ be such that $\psi_{j}\left(\tau_{j}\right)=w_{0}, j \in\{1,2\}$. Then, in virtue of (6.5) and (6.10) and the result of the previous paragraph on the well-definedness of $\hat{f}_{0}$, the well-defined property of $\hat{f}$ is reduced to showing that

$$
\hat{f}_{1}\left(\phi(t), \tau_{1}\right)=\hat{f}_{2}\left(\phi(t), \tau_{2}\right)
$$

for all $t \in E$ and all $\epsilon$-candidates $(\phi, \Gamma)$ for $(\phi(t), A, D)$, such that

$$
\omega(t, \Gamma, A)<\epsilon:=1-\max _{j \in\{1,2\}}\left\{\omega\left(\tau_{1}, \Delta_{1}, E\right), \omega\left(\tau_{2}, \Delta_{2}, E\right)\right\} .
$$

Observe that (6.11) follows from an argument based on Part 2) of Lemma 4.5. Hence, $\hat{f}$ is well-defined on $\widehat{W}$.

As in (6.8), for all $0<\delta<1$ let

$$
\begin{array}{ll}
A_{\delta}:=\{z \in D: \omega(z, A, D)<\delta\}, & B_{\delta}:=\{w \in G: \omega(w, B, G)<\delta\}, \\
D_{\delta}:=\{z \in D: \omega(z, A, D)<1-\delta\}, & G_{\delta}:=\{w \in G: \omega(w, B, G)<1-\delta\} .
\end{array}
$$

Now we combine (6.8) and (6.12) and the result that $\hat{f}_{0}$, given by (6.7), is welldefined and holomorphic on $\widehat{W}_{0}$, and the result that $\hat{f}$ is well-defined on $\widehat{W}$. Consequently, we obtain that

$$
\hat{f}(\cdot, w) \in \mathcal{O}\left(D_{\delta}, \mathbb{C}\right), \quad w \in B_{\delta}, 0<\delta<1 .
$$

Since the formula (6.5) for $\hat{f}$ is symmetric in two variables $(z, w)$, one also gets that

$$
\hat{f}(z, \cdot) \in \mathcal{O}\left(G_{\delta}, \mathbb{C}\right), \quad z \in A_{\delta}, 0<\delta<1 .
$$


Since by (6.12),

$$
\widehat{W}=\bigcup_{0<\delta<1} A_{\delta} \times G_{\delta}=\bigcup_{0<\delta<1} D_{\delta} \times B_{\delta},
$$

it follows from the previous conclusions that, for all points $(z, w) \in \widehat{W}$, there is an open neighborhood $U$ of $z$ (respectively $V$ of $w$ ) such that $f \in \mathcal{O}_{s}\left(\mathbb{X}^{\mathrm{O}}(U, V ; U, V), \mathbb{C}\right)$. By the classical Hartogs extension theorem, $f \in \mathcal{O}(U \times V, \mathbb{C})$. Hence, $\hat{f} \in \mathcal{O}(\widehat{W}, \mathbb{C})$.

On the other hand, it follows from (6.5) and the estimate in Theorem 4.3 that

$$
|\hat{f}|_{\widehat{W}} \leq|f|_{\bar{W}}
$$

This completes Step 1.

Step 2. $\left.f\right|_{\bar{A} \times \bar{B}} \in \mathcal{C}(\bar{A} \times \bar{B}, \mathbb{C})$.

Proof of Step 2. Using the hypotheses we only need to check the continuity of $\left.f\right|_{\bar{A} \times \bar{B}}$ at every point $\left(a_{0}, w_{0}\right) \in \bar{A} \times(G \cap \bar{B})$ and at every point $\left(z_{0}, b_{0}\right) \in$ $(D \cap \bar{A}) \times \bar{B}$. We will verify the first assertion. To do this let $\left(a_{k}\right)_{k=1}^{\infty} \subset \bar{A}$ and $\left(w_{k}\right)_{k=1}^{\infty} \subset(G \cap \bar{B})$ such that $\lim _{k \rightarrow \infty} a_{k}=a_{0}$ and $\lim _{k \rightarrow \infty} w_{k}=w_{0}$. We need to show that

$$
\lim _{k \rightarrow \infty} f\left(a_{k}, w_{k}\right)=f\left(a_{0}, w_{0}\right)
$$

Since $\left.f\right|_{\bar{W}}$ is locally bounded, we may choose an open connected neighborhood $V$ of $w_{0}$ such that $\sup _{k>1}\left|f\left(a_{k}, \cdot\right)\right|_{V}<\infty$. Consequently, by Montel's theorem, there is a sequence $\left(k_{p}\right)_{p=1}^{\infty}$ such that $\left(f\left(a_{k_{p}}, \cdot\right)\right)$ converges uniformly on compact subsets of $V$ to a function $g \in \mathcal{O}(V)$. Equality (6.14) is reduced to showing that $g=f\left(a_{0}, \cdot\right)$ on $V$. Since $f \in \mathcal{C}_{s}(\bar{W}, \mathbb{C})$, we deduce that $f\left(a_{0}, \cdot\right)=g$ on $B \cap V$. On the other hand, $B \cap V$ is non locally pluripolar because $B$ is locally pluriregular and $w_{0} \in \bar{B}$. Hence, we conclude by the uniqueness principle that $g=f\left(a_{0}, \cdot\right)$ on $V$.

Step 3. $\hat{f}$ admits $\mathcal{A}$-limit $f(\zeta, \eta)$ at all points $(\zeta, \eta) \in W$.

Proof of Step 3. To this end we only need to prove that

$$
\left(\mathcal{A}-\lim \sup \left|\hat{f}-f\left(\zeta_{0}, \eta_{0}\right)\right|\right)\left(\zeta_{0}, \eta_{0}\right)<\epsilon_{0}
$$

for an arbitrary fixed point $\left(\zeta_{0}, \eta_{0}\right) \in W$ and an arbitrary fixed $0<\epsilon_{0}<1$. Suppose without loss of generality that

$$
|f|_{W} \leq \frac{1}{2}
$$

First consider $\left(\zeta_{0}, \eta_{0}\right) \in A \times B$. Since $f \in \mathcal{C}(\bar{A} \times \bar{B}, \mathbb{C})$, one may find an open neighborhood $U$ of $\zeta_{0}$ in $\mathbb{C}^{n}$ (respectively $V$ of $\eta_{0}$ in $\mathbb{C}^{m}$ ) so that

$$
\left|f-f\left(\zeta_{0}, \eta_{0}\right)\right|_{(\bar{A} \cap U) \times(\bar{B} \cap V)}<\frac{\epsilon_{0}^{2}}{4} .
$$


Consider the open sets

$$
\begin{aligned}
D^{\prime} & :=\left\{z \in D: \omega(z, A \cap U, D)<\frac{1}{2}\right\} \quad \text { and } \\
G^{\prime} & :=\left\{w \in G: \omega(w, B \cap V, G)<\frac{1}{2}\right\} .
\end{aligned}
$$

In virtue of (6.16)-(6.18), an application of Theorem 3.6 gives that

$$
\left|f(\zeta, w)-f\left(\zeta, \eta_{0}\right)\right| \leq\left(\frac{\epsilon_{0}^{2}}{4}\right)^{1-\omega(w, B \cap V, G)} \leq \frac{\epsilon_{0}}{2}, \quad \zeta \in A \cap U, w \in G^{\prime}
$$

Hence,

$$
\left|f-f\left(\zeta_{0}, \eta_{0}\right)\right|_{\mathbb{X}\left(\bar{A} \cap U, \bar{B} \cap V ; D^{\prime}, G^{\prime}\right)} \leq \frac{\epsilon_{0}}{2}
$$

Consider the function $g: \mathbb{X}\left(\bar{A} \cap U, \bar{B} \cap V ; D^{\prime}, G^{\prime}\right) \longrightarrow \mathbb{C}$, given by

$$
g(z, w):=f(z, w)-f\left(\zeta_{0}, \eta_{0}\right)
$$

Applying the result of Step 1 , we can construct a function $\hat{g} \in \mathcal{O}(\widehat{\mathbb{X}}(A \cap U, B \cap$ $\left.\left.V ; D^{\prime}, G^{\prime}\right), \mathbb{C}\right)$ from $g$ in exactly the same way as we obtain $\hat{f} \in \mathcal{O}(\widehat{W}, \mathbb{C})$ from $f$. Moreover, combining (6.5) and (6.20), we see that

$$
\hat{g}=\hat{f}-f\left(\zeta_{0}, \eta_{0}\right) \quad \text { on } \quad \widehat{\mathbb{X}}\left(A \cap U, B \cap V ; D^{\prime}, G^{\prime}\right) .
$$

On the other hand, it follows from formula (6.20), estimate (6.19), and estimate (6.13) that

$$
|\hat{g}|_{\widehat{\mathbb{X}}\left(A \cap U, B \cap V ; D^{\prime}, G^{\prime}\right)} \leq \frac{\epsilon_{0}}{2} .
$$

This, combined with (6.21) and (6.18), implies that

$$
\left(\mathcal{A}-\lim \sup \left|\hat{f}(z, w)-f\left(\zeta_{0}, \eta_{0}\right)\right|\right)\left(\zeta_{0}, \eta_{0}\right) \leq \frac{\epsilon_{0}}{2}
$$

Hence, (6.15) follows. In summary, we have shown that $\mathcal{A}-\lim \hat{f}=f$ on $A \times B$. Now it remains to consider $\left(\zeta_{0}, \eta_{0}\right) \in A \times G$. Using the last limit and arguing as in Step 2, one can show that $\mathcal{A}-\lim \hat{f}\left(\zeta_{0}, \eta_{0}\right)=f\left(\zeta_{0}, \eta_{0}\right)$.

Step 4. Proof of the uniqueness of $\hat{f}$ and (6.1).

Proof of Step 4. To prove the uniqueness of $\hat{f}$ suppose that $\hat{g} \in \mathcal{O}(\widehat{W}, \mathbb{C})$ is a bounded function which admits $\mathcal{A}$-limit $f(\zeta, \eta)$ at all points $(\zeta, \eta) \in W$. Fix an 
arbitrary point $\left(z_{0}, w_{0}\right) \in \widehat{W}$, it suffices to show that $\hat{f}\left(z_{0}, w_{0}\right)=\hat{g}\left(z_{0}, w_{0}\right)$. Observe that both functions $\hat{f}\left(z_{0}, \cdot\right)$ and $\hat{g}\left(z_{0}, \cdot\right)$ are bounded and holomorphic on the $\delta$-level set of $G$ relative to $B$ :

$$
G_{\delta, B}:=\left\{w \in G: \omega(w, B, G)<1-\omega\left(z_{0}, A, D\right)\right\}
$$

where $\delta:=\omega\left(z_{0}, A, D\right)$. On the other hand, they admit $\mathcal{A}$-limit $f\left(z_{0}, \eta\right)$ at all points $\eta \in B$. Consequently, applying Proposition 3.5 and Theorem 3.7 yields that $\hat{f}\left(z_{0}, \cdot\right)=\hat{g}\left(z_{0}, \cdot\right)$ on $G_{\delta, B}$. Hence, $\hat{f}\left(z_{0}, w_{0}\right)=\hat{g}\left(z_{0}, w_{0}\right)$.

To prove (6.1) fix an arbitrary point $\left(z_{0}, w_{0}\right) \in \widehat{W}$. For every $\eta \in B$, applying Theorem 3.6 to $\log |f(\cdot, \eta)|$ defined on $D$, we obtain that

$$
\left|f\left(z_{0}, \eta\right)\right| \leq|f|_{A \times B}^{1-\omega\left(z_{0}, A, D\right)}|f|_{W}^{\omega\left(z_{0}, A, D\right)} .
$$

Applying Theorem 3.6 again to $\log \left|\hat{f}\left(z_{0}, \cdot\right)\right|$ defined on $G_{\delta, B}$ of the preceeding paragraph, one gets that

$$
\left|\hat{f}\left(z_{0}, w_{0}\right)\right| \leq\left|f\left(z_{0}, \cdot\right)\right|_{B}^{1-\omega\left(w_{0}, B, G\right)}|\hat{f}|_{\widehat{W}}^{\omega\left(w_{0}, B, G\right)} .
$$

Inserting (6.13) and (6.22) into the right hand side of the latter estimate, (6.1) follows. Hence Step 4 is finished.

This completes the proof.

In the sequel we will need the following refined version of Theorem 6.1 .

Theorem 6.2. Let $D \subset \mathbb{C}^{n}, G \subset \mathbb{C}^{m}$ be bounded open sets. $D$ (respectively $G)$ is equipped with a system of approach regions $\left(\mathcal{A}_{\alpha}(\zeta)\right)_{\zeta \in \bar{D}, \alpha \in I_{\zeta}}$ (respectively $\left.\left(\mathcal{A}_{\alpha}(\eta)\right)_{\eta \in \bar{G}}, \alpha \in I_{\eta}\right)$. Let $A, A_{0}$ (respectively $B, B_{0}$ ) be subsets of $\bar{D}$ (respectively $\bar{G})$ such that $A_{0}$ and $B_{0}$ are locally pluriregular and that $\bar{A}_{0} \subset A^{*}$ and $\bar{B}_{0} \subset B^{*}$. Put

$$
W:=\mathbb{X}(A, B ; D, G) \quad \text { and } \quad W_{0}:=\mathbb{X}\left(A_{0}, B_{0} ; D, G\right) .
$$

Then, for every bounded function $f: W \longrightarrow \mathbb{C}$ which satisfies the following conditions:

- $f \in \mathcal{C}_{S}(W, \mathbb{C}) \cap \mathcal{O}_{s}\left(W^{o}, \mathbb{C}\right)$;

- $\left.f\right|_{A \times B}$ is continuous at all points of $(A \cap \partial D) \times(B \cap \partial G)$,

there exists a unique bounded function $\hat{f} \in \mathcal{O}\left(\widehat{W}_{0}, \mathbb{C}\right)$ which admits $\mathcal{A}$-limit $f(\zeta, \eta)$ at all points $(\zeta, \eta) \in W_{0}$. Moreover,

$$
|\hat{f}(z, w)| \leq|f|_{A_{0} \times B_{0}}^{1-\omega\left(z, A_{0}, D\right)-\omega\left(w, B_{0}, G\right)}|f|_{W}^{\omega\left(z, A_{0}, D\right)+\omega\left(w, B_{0}, G\right)}, \quad(z, w) \in \widehat{W}_{0} .
$$


Proof. Using the hypotheses and applying Part 1) of Theorem 7.2 below we can extend $f$ to a locally bounded function (still denoted by) $f$ defined on $\mathbb{X}\left(A^{*}, B^{*}, D, G\right)$ such that $f \in \mathcal{O}_{s}\left(\mathbb{X}^{\mathrm{O}}\left(A^{*}, B^{*}, D, G\right), \mathbb{C}\right)$ and that $\left.f\right|_{\mathbb{X}\left(A^{*} \cap D, B^{*} \cap G ; D, G\right)}$ is continuous. Therefore, the newly defined function $f$ satisfies

$$
f(a, b):=\lim _{k \rightarrow \infty} f\left(a_{k}, b\right)
$$

where $(a, b)$ is an arbitrary point of $A^{*} \times\left(G \cup B^{*}\right)$ and $\left(a_{k}\right)_{k=1}^{\infty} \subset A^{*}$ is an arbitrary sequence with $\lim _{k \rightarrow \infty} a_{k}=a$. Since $\left.f\right|_{W}$ is bounded, it follows that the newly defined function $f$ is also bounded. In virtue of the definition of $A^{*}$ and $B^{*}$ we have

$$
\partial D \cap A=\partial D \cap A^{*} \quad \text { and } \quad \partial G \cap B=\partial G \cap B^{*} .
$$

Using the second $\bullet$ in the hypotheses and formula (6.24) we see that $\left.f\right|_{A^{*} \times B^{*}}$ is continuous at all points all $(\partial D \cap A) \times(\partial G \cap B)$. Consequently, arguing as in the proof of Step 2 of Theorem 6.1 and using (6.25), we can show that $f \in \mathcal{C}\left(A^{*} \times\right.$ $\left.B^{*}, \mathbb{C}\right)$. In summary, the newly defined function $f$ which is defined and bounded on $\mathbb{X}\left(A^{*}, B^{*}, D, G\right)$ satisfies

$$
f \in \mathcal{O}_{s}\left(\mathbb{X}^{\mathrm{o}}\left(A^{*}, B^{*}, D, G\right), \mathbb{C}\right) \text { and } f \in \mathcal{C}\left(A^{*} \times B^{*}, \mathbb{C}\right) .
$$

Observe that $f$ is only separately continuous on $\mathbb{X}(A, B ; D, G)$, but it is not necessarily so on the cross $\mathbb{X}\left(A^{*}, B^{*}, D, G\right)$. However, we will show that one can adapt the argument of Theorem 6.1 in order to prove Theorem 6.2.

We define $\hat{f}$ at an arbitrary point $\left(z_{0}, w_{0}\right) \in \widehat{W}_{0}$ as follows: Let $\epsilon>0$ be such that

$$
\omega\left(z_{0}, A_{0}, D\right)+\omega\left(w_{0}, B_{0}, G\right)+2 \epsilon<1 .
$$

By Theorem 3.8 and Definition 3.9, there is an $\epsilon$-candidate $(\phi, \Gamma)$ (respectively $(\psi, \Delta))$ for $\left(z_{0}, A_{0}, D\right)$ (respectively $\left.\left(w_{0}, B, G\right)\right)$. To conclude the proof we only need to prove that the function $f_{\phi, \psi}$, defined by

$$
f_{\phi, \psi}(t, \tau):=f(\phi(t), \psi(\tau)), \quad(t, \tau) \in \mathbb{X}(\Gamma, \Delta ; E, E),
$$

satisfies the hypotheses of Theorem 4.3. Indeed, having proved this assertion, the proof will follow along the same lines as those given in Theorem 6.1. This assertion is again reduced to showing that for each fixed $t \in \Gamma$, the function $f_{\phi, \psi}(t, \cdot)$ admits the angular limit $f(\phi(t), \psi(\tau))$ for every point $\tau \in \Delta$. We will prove the last claim.

Using the first $\bullet$ and Theorem 3.8, we see that for every $a \in A$, the function $f(a, \psi(\cdot)) \in \mathcal{O}(E, \mathbb{C})$ admits the angular limit $f(a, \psi(\tau))$ for every point $\tau \in \Delta$. Next, using the hypothesis $\bar{A}_{0} \subset A^{*}$ we may choose a sequence $\left(a_{k}\right)_{k=1}^{\infty} \subset$ $A \cap A^{*}$ such that $\lim _{k \rightarrow \infty} a_{k}=\phi(t) \in \bar{A}_{0}$. Observe from (6.26) that for every $k$ the uniformly bounded function $f\left(a_{k}, \psi(\cdot)\right) \in \mathcal{O}(E, \mathbb{C})$ admits the angular limit $f\left(a_{k}, \psi(\tau)\right)$ and that $\lim _{k \rightarrow \infty} f\left(a_{k}, \psi(\tau)\right)=f(\phi(t), \psi(\tau))$ for every point $\tau \in \Delta$. Consequently, by the Khinchin-Ostrowski theorem (see [11, Theorem 4, page 397$]$ ), the above claim follows. 


\section{Preparatory results}

The first result of this section shows that the two definitions of plurisubharmonic measure $\widetilde{\omega}(\cdot, A, D)$, given respectively in Definition 2.3 and in Subsection 2.1 of [28], coincide in the case when $A \subset D$.

Proposition 7.1. Let $X$ be a complex manifold and $D \subset X$ an open set. $D$ is equipped with the canonical system $\mathcal{A}$ of approach regions. Let $A$ be a subset of $D$. Then $\widetilde{\omega}(z, A, D)=\omega\left(z, A^{*}, D\right)$.

Proof. Let $P \in \mathcal{E}(A)$. Then by Definition 2.3, $P \subset A^{*}$ and $P$ is locally pluriregular. Hence, $P \subset \mathcal{\sim}_{(}\left(A^{*}\right)^{*}=A^{*}$. Since $P \in \underset{\sim}{\mathcal{E}}(A)$ is arbitrary, it follows from Definition 2.3 that $\widetilde{A}$ is locally pluriregular and $\widetilde{A} \subset A^{*}$. In particular, $(\widetilde{A})^{*} \subset A^{*}$ and

$$
\widetilde{\omega}(z, A, D)=\omega(z, \widetilde{A}, D) \geq \omega\left(z, A^{*}, D\right) .
$$

In the sequel we will show that

$$
A^{*} \subset(\widetilde{A})^{*} \text {. }
$$

Taking (7.2) for granted, we have that $A^{*}=(\widetilde{A})^{*}$. Consequently,

$$
\widetilde{\omega}(z, A, D)=\omega(z, \widetilde{A}, D) \leq \omega\left(z, A^{*}, D\right) .
$$

This, coupled with (7.1), completes the proof.

To prove (7.2) fix an arbitrary point $a \in A^{*}$ and an arbitrary but sufficiently small neighborhood $U \subset X$ of $a$ such that $U$ is biholomorphic to a bounded open set in $\mathbb{C}^{n}$, where $n$ is the dimension of $X$ at $a$. Since $A^{*}$ is a Borel subset of $D$, Theorem 8.5 in [7] provides a subset $P \subset A^{*} \cap U$ of type $\mathcal{F}_{\sigma}{ }^{10}$ such that

$$
\omega(z, P, U)=\omega\left(z, A^{*} \cap U, U\right), \quad z \in U .
$$

Write $P=\bigcup_{n \geq 1} P_{n}$, where $P_{n}$ is closed. Observe that $P_{n} \cap P_{n}^{*}$ is locally pluriregular, $P_{n} \backslash\left(P_{n} \cap P_{n}^{*}\right)$ is locally pluripolar and $\overline{P_{n} \cap P_{n}^{*}} \subset P_{n} \subset A^{*} \cap P$. Consequently, $\bigcup_{n \geq 1}\left(P_{n} \cap P_{n}^{*}\right) \subset \widetilde{A} \cap P$ and $P \backslash \bigcup_{n \geq 1}\left(P_{n} \cap P_{n}^{*}\right)$ is locally pluripolar. This implies that

$$
\omega(z, \tilde{A} \cap U, U) \leq \omega\left(z, \bigcup_{n \geq 1}\left(P_{n} \cap P_{n}^{*}\right), U\right)=\omega(z, P, U),
$$

where the equality holds by applying Lemma 3.5.3 in [18] and by using the fact that $U$ is biholomorphic to a bounded open set in $\mathbb{C}^{n}$. This, combined with (7.3) and the assumption $a \in A^{*}$, implies that $\omega(a, \widetilde{A} \cap U, U)=0$. Thus (7.2) follows.

The main purpose of this and the next sections is to generalize Theorem 6.1 to the case where the "target space" $Z$ is an arbitrary complex analytic space possessing the Hartogs extension property.

10 This means that $P$ is a countable (or finite) union of relatively closed subsets of $U$. 
Theorem 7.2. Let $D \subset \mathbb{C}^{n}, G \subset \mathbb{C}^{m}$ be two bounded open sets. $D$ (respectively $G)$ is equipped with the canonical system of approach regions. Let $Z$ be a complex analytic space possessing the Hartogs extension property. Let $A$ (respectively $B)$ be a subset of $D$ (respectively $G)$. Put $W:=\mathbb{X}(A, B ; D, G)$ and $\widehat{W}:=$ $\widehat{\mathbb{X}}(A, B ; D, G)$. Let $f \in \mathcal{O}_{s}\left(W^{o}, Z\right)$.

1) Then $f$ extends to a mapping (still denoted by) $f$ defined on $\mathbb{X}^{\circ}\left(A \cup A^{*}, B \cup\right.$ $\left.B^{*} ; D, G\right)$ such that $f$ is separately holomorphic on $\mathbb{X}^{o}\left(A \cup A^{*}, B \cup B^{*} ; D, G\right)$ and that $\left.f\right|_{\mathbb{X}^{o}}\left(A^{*}, B^{*} ; D, G\right)$ is continuous.

2) Suppose in addition that $A$ and $B$ are locally pluriregular. Then $f$ extends to $a$ unique mapping $\hat{f} \in \mathcal{O}(\widehat{W}, Z)$ such that $\hat{f}=f$ on $W$.

Proof. This result has already been proved in Théorème 2.2.4 in [5] starting from Proposition 3.2.1 therein. In the latter proposition Alehyane and Zeriahi make use of the method of doubly orthogonal bases of Bergman type. We can avoid this method by simply replacing every application of this proposition by Theorem 6.1. Keeping this change in mind and using Proposition 7.1, the remaining part of the proof follows along the same lines as that of Théorème 2.2.4 in [5].

Theorem 7.3. Let $D, G$ be complex manifolds, and let $A \subset D, B \subset G$ be open subsets. Let $Z$ be a complex analytic space possessing the Hartogs extension property. Put $W:=\mathbb{X}(A, B ; D, G)$ and $\widehat{W}:=\widehat{\mathbb{X}}(A, B ; D, G)$. Then for any mapping $f \in \mathcal{O}_{s}(W, Z)$, there is a unique mapping $\hat{f} \in \mathcal{O}(\widehat{W}, Z)$ such that $\hat{f}=f$ on $W$.

Proof. It has already been proved in Theorem 5.1 of [28]. The only places where the method of doubly orthogonal bases of Bergman type is involved is the applications of Théorème 2.2.4 in [5]. As we already pointed out in Theorem 7.2, one can avoid this method by using Theorem 6.1 instead.

We are ready to formulate a slight generalization of Theorems 6.2 and 7.2.

Theorem 7.4. Let $D \subset \mathbb{C}^{n}, G \subset \mathbb{C}^{m}$ be bounded open sets. $D$ (respectively $G)$ is equipped with a system of approach regions $\left(\mathcal{A}_{\alpha}(\zeta)\right)_{\zeta \in \bar{D}, \alpha \in I_{\zeta}}$ (respectively $\left.\left(\mathcal{A}_{\beta}(\eta)\right)_{\eta \in \bar{G}}, \beta \in I_{\eta}\right)$. Let $A$ and $A_{0}$ (respectively $B$ and $B_{0}$ ) be two subsets of $\bar{D}$ (respectively $\bar{G}$ ) such that $A_{0}$ and $B_{0}$ are locally pluriregular and that $\bar{A}_{0} \subset A^{*}$ and $\bar{B}_{0} \subset B^{*}$. Let $Z$ be a complex analytic space possessing the Hartogs extension property. Put

$$
W:=\mathbb{X}(A, B ; D, G) \quad \text { and } \quad W_{0}:=\mathbb{X}\left(A_{0}, B_{0} ; D, G\right)
$$

Then, for every bounded mapping $f: W \longrightarrow Z$ which satisfies the following conditions:

- $f \in \mathcal{C}_{s}(W, Z) \cap \mathcal{O}_{s}\left(W^{o}, Z\right)$;

- $\left.f\right|_{A \times B}$ is continuous at all points of $(A \cap \partial D) \times(B \cap \partial G)$, 
there exists a unique bounded mapping $\hat{f} \in \mathcal{O}\left(\widehat{W}_{0}, \mathbb{C}\right)$ which admits $\mathcal{A}$-limit $f(\zeta, \eta)$ at all points $(\zeta, \eta) \in W_{0}$.

Proof. Since $f$ is bounded, one may find an open neighborhood $U$ of $f(\bar{W})$ in $Z$ and a holomorphic embedding $\phi$ of $U$ into the polydisc $E^{k}$ of $\mathbb{C}^{k}$ such that $\phi(U)$ is an analytic set in $E^{k}$. Now we are able to apply Theorem 6.2 to the mapping $\phi \circ f$ : $\bar{W} \longrightarrow \mathbb{C}^{k}$. Consequently, one obtains a unique bounded mapping $F \in \mathcal{O}\left(\widehat{W}, \mathbb{C}^{k}\right)$ which admits $\mathcal{A}$-limit $(\phi \circ f)(\zeta, \eta)$ at all points $(\zeta, \eta) \in W$. Using estimate (6.23) one can show that $F \in \mathcal{O}\left(\widehat{W}, E^{k}\right)$. Now using Theorem 3.7 it is not difficult to see that $F(\widehat{W}) \subset \phi(U)$. Consequently, one can define the desired extension mapping $\hat{f}$ as follows:

$$
\hat{f}(z, w):=\left(\phi^{-1} \circ F\right)(z, w), \quad(z, w) \in \widehat{W} .
$$

The following uniqueness theorem for holomorphic mappings generalizes Theorem 3.7 .

Theorem 7.5. Let $X$ be a complex manifold, $D \subset X$ an open subset and $Z a$ complex analytic space. Suppose that $D$ is equipped with a system of approach regions $\left(\mathcal{A}_{\alpha}(\zeta)\right)_{\zeta \in \bar{D}, \alpha \in I_{\zeta}}$. Let $A \subset \bar{D}$ be a locally pluriregular set. Let $f_{1}, f_{2}$ : $D \cup A \longrightarrow Z$ be locally bounded mappings such that $\left.f_{1}\right|_{D},\left.f_{2}\right|_{D} \in \mathcal{O}(D, Z)$ and $\mathcal{A}-\lim f_{1}=\mathcal{A}-\lim f_{2}$ on $A$. Then $f_{1}(z)=f_{2}(z)$ for all $z \in D$ such that $\omega(z, A, D) \neq 1$.

We leave the proof to the interested reader. Finally, we conclude this section with the following Gluing Lemma.

Lemma 7.6. Let $\mathcal{D}$ and $\mathcal{G}$ be open subsets of some complex manifolds and $Z$ a complex analytic space. Suppose that $\mathcal{D}$ (respectively $\mathcal{G}$ ) is equipped with a system of approach regions $\left(\mathcal{A}_{\alpha}(\zeta)\right)_{\zeta \in \overline{\mathcal{D}}, \alpha \in I_{\zeta}}$ (respectively $\left.\left(\mathcal{A}_{\beta}(\eta)\right)_{\eta \in \overline{\mathcal{G}}, \beta \in I_{\eta}}\right)$. Let $\left(\mathcal{D}_{k}\right)_{k=k_{0}}^{\infty}$ (respectively $\left(\mathcal{G}_{k}\right)_{k=k_{0}}^{\infty}$ ) be a family of open subsets of $\mathcal{D}$ (respectively $\mathcal{G}$ ) equipped with the induced system of approach regions. Let $\left(\mathcal{P}_{k}\right)_{k=k_{0}}^{\infty}$ (respectively $\left.\left(\mathcal{Q}_{k}\right)_{k=k_{0}}^{\infty}\right)$ be a family of locally pluriregular subsets of $\overline{\mathcal{D}}$ (respectively $\overline{\mathcal{G}}$ ). Suppose, in addition, that

(i) $\mathcal{P}_{k} \subset \mathcal{P}_{k_{0}}, \mathcal{D}_{k_{0}} \subset \mathcal{D}_{k}$, and $\mathcal{P}_{k}$ is locally pluriregular relative to $\mathcal{D}_{k_{0}}$. Similarly, $\mathcal{Q}_{k} \subset \mathcal{Q}_{k_{0}}, \mathcal{G}_{k_{0}} \subset \mathcal{G}_{k}$, and $\mathcal{Q}_{k}$ is locally pluriregular relative to $\mathcal{G}_{k_{0}}$.

(ii) There are a family of locally bounded mappings $\left(f_{k}\right)_{k=k_{0}}^{\infty}$ such that $f_{k}$ : $\mathbb{X}^{o}\left(\mathcal{P}_{k}, \mathcal{Q}_{k} ; \mathcal{D}_{k}, \mathcal{G}_{k}\right) \longrightarrow Z$ verifies $f_{k}=f_{k_{0}}$ on $\mathbb{X}^{o}\left(\mathcal{P}_{k}, \mathcal{Q}_{k} ; \mathcal{D}_{k_{0}}, \mathcal{G}_{k_{0}}\right)$, and a family of holomorphic mappings $\left(\hat{f}_{k}\right)_{k=k_{0}}^{\infty}$ such that $\hat{f}_{k} \in \mathcal{O}\left(\widehat{\mathbb{X}}\left(\mathcal{P}_{k}, \mathcal{Q}_{k} ; \mathcal{D}_{k}, \mathcal{G}_{k}\right), Z\right)$, and

$$
\left(\mathcal{A}-\lim \hat{f}_{k}\right)(z, w)=f_{k}(z, w), \quad(z, w) \in \mathbb{X}^{o}\left(\mathcal{P}_{k}, \mathcal{Q}_{k} ; \mathcal{D}_{k_{0}}, \mathcal{G}_{k_{0}}\right) .
$$

(iii) There are open subsets $U$ of $\mathcal{D}$ and $V$ of $\mathcal{G}$ such that $\widetilde{\omega}\left(z, \mathcal{P}_{k}, \mathcal{D}_{k_{0}}\right)+$ $\tilde{\omega}\left(w, \mathcal{Q}_{k}, \mathcal{G}_{k_{0}}\right)<1$ for all $(z, w) \in U \times V$ and $k \geq k_{0}$. 
Then $\hat{f}_{k}(z, w)=\hat{f}_{k_{0}}(z, w)$ for all $(z, w) \in U \times V$ and $k \geq k_{0}$.

Proof. By (iii), we have that

$$
U \times V \subset H:=\widehat{\mathbb{X}}\left(\mathcal{P}_{k}, \mathcal{Q}_{k} ; \mathcal{D}_{k_{0}}, \mathcal{G}_{k_{0}}\right) .
$$

On the other hand, using (i) we see that

$$
H \subset \widehat{\mathbb{X}}\left(\mathcal{P}_{k}, \mathcal{Q}_{k} ; \mathcal{D}_{k}, \mathcal{G}_{k}\right) \cap \widehat{\mathbb{X}}\left(\mathcal{P}_{k_{0}}, \mathcal{Q}_{k_{0}} ; \mathcal{D}_{k_{0}}, \mathcal{G}_{k_{0}}\right) .
$$

Fix arbitrary $\left(z_{0}, w_{0}\right) \in H$ and $k \geq k_{0}$. Observe that both mappings $\hat{f}_{k}\left(\cdot, w_{0}\right)$ and $\hat{f}_{k_{0}}\left(\cdot, w_{0}\right)$ are defined on $\left\{z \in \mathcal{D}_{k_{0}}: \omega\left(z, \mathcal{P}_{k}, \mathcal{D}_{k_{0}}\right)<1-\omega\left(w_{0}, \mathcal{Q}_{k}, \mathcal{G}_{k_{0}}\right)\right\}$. Using (ii) and Proposition 3.5, we may apply Theorem 7.5 to these mappings and conclude that $\hat{f}_{k}\left(z_{0}, w_{0}\right)=\hat{f}_{k_{0}}\left(z_{0}, w_{0}\right)$.

\section{Local and semi-local versions of Theorem A}

The aim of this section is to generalize Theorem 6.2 to some cases where the "target space" $Z$ is a complex analytic space possessing the Hartogs extension property. Our philosophy is the following: we first apply Theorem 6.2 locally in order to obtain various local extension mappings, then we glue them together. The gluing process needs the following

Definition 8.1. Let $\mathcal{M}$ be a complex manifold and $Z$ a complex space. Let $\left(U_{j}\right)_{j \in J}$ be a family of open subsets of $\mathcal{M}$, and $\left(f_{j}\right)_{j \in J}$ a family of mappings such that $f_{j} \in \mathcal{O}\left(U_{j}, Z\right)$. We say that the family $\left(f_{j}\right)_{j \in J}$ is collective if, for any $j, k \in J$, $f_{j}=f_{k}$ on $U_{j} \cap U_{k}$. The unique holomorphic mapping $f: \bigcup_{j \in J} U_{j} \longrightarrow Z$, defined by $f:=f_{j}$ on $U_{j}, j \in J$, is called the collected mapping of $\left(f_{j}\right)_{j \in J}$.

We arrive at the following local version of Theorem A.

Theorem 8.2. Let $D \subset \mathbb{C}^{p}, G \subset \mathbb{C}^{q}$ be bounded open sets and $Z$ a complex analytic space possessing the Hartogs extension property. $D$ (respectively $G$ ) is equipped with a system of approach regions $\left(\mathcal{A}_{\alpha}(\zeta)\right)_{\zeta \in \bar{D}, \alpha \in I_{\zeta}}$ (respectively $\left(\mathcal{A}_{\beta}(\eta)\right)_{\eta \in \bar{G}, \beta \in I_{\eta}}$ ). Let $A, A_{0}$ (respectively $B, B_{0}$ ) be subsets of $\bar{D}$ (respectively $\bar{G}$ ) such that $A_{0}$ and $B_{0}$ are locally pluriregular and that $\bar{A}_{0} \subset A^{*}$ and $\bar{B}_{0} \subset B^{*}$. Put

$$
W:=\mathbb{X}(A, B ; D, G) \quad \text { and } \quad W_{0}:=\mathbb{X}\left(A_{0}, B_{0} ; D, G\right) .
$$

Then, for every mapping $f: W \longrightarrow Z$ which satisfies the following conditions:

- $f \in \mathcal{C}_{s}(W, Z) \cap \mathcal{O}_{s}\left(W^{o}, Z\right)$;

- $f$ is locally bounded along $\mathbb{X}(A \cap \partial D, B \cap \partial G ; D, G)$;

- $\left.f\right|_{A \times B}$ is continuous at all points of $(A \cap \partial D) \times(B \cap \partial G)$,

there exists a unique mapping $\hat{f} \in \mathcal{O}\left(\widehat{W}_{0}, Z\right)$ which admits $\mathcal{A}$-limit $f(\zeta, \eta)$ at all points $(\zeta, \eta) \in W_{0}$. 
Theorem 8.2 generalizes Theorem 6.2 to the case where the "target space" $Z$ is an arbitrary complex analytic space possessing the Hartogs extension property. Since the proof is somewhat technical, the reader may skip it at the first reading.

Proof. Recall that for $a \in \mathbb{C}^{k}$ and $r>0, \mathbb{B}(a, r)$ denotes the open ball centered at $a$ with radius $r$. For $0<\delta<1$ and $0<r$ put

$$
\begin{array}{ll}
D_{a, \delta, r}:=\left\{z \in D \cap \mathbb{B}(a, r): \omega\left(A_{0} \cap \mathbb{B}(a, r), D \cap \mathbb{B}(a, r)\right)<\delta\right\}, & a \in \bar{A}_{0}, \\
G_{b, \delta, r}:=\left\{w \in G \cap \mathbb{B}(b, r): \omega\left(B_{0} \cap \mathbb{B}(b, r), G \cap \mathbb{B}(b, r)\right)<\delta\right\}, & b \in \bar{B}_{0} .
\end{array}
$$

Applying Part 1) of Theorem 7.2 and using the hypotheses on $f$, we see that $f$ extends to a mapping defined on $\mathbb{X}\left(A \cup A^{*}, B \cup B^{*} ; D, G\right)$ such that $f$ is separately holomorphic on $\mathbb{X}^{\mathrm{o}}\left(A \cup A^{*}, B \cup B^{*} ; D, G\right)$ and that $\left.f\right|_{\mathbb{X}\left(A^{*}, B^{*} ; D, G\right)}$ is locally bounded.

Therefore, using the compactness of $\bar{A}_{0}$ and $\bar{B}_{0}$, one may find a real number $r_{0}>0$ such that

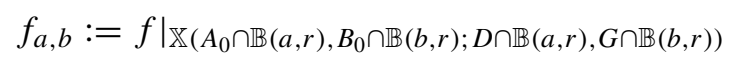

is bounded for all $0<r \leq r_{0}$ and $a \in \bar{A}_{0}, b \in \bar{B}_{0}$. Applying Theorem 7.4 to $f_{a, b}$, one obtains a mapping

$$
\hat{f}_{a, b} \in \mathcal{O}\left(\widehat{\mathbb{X}}\left(A_{0} \cap \mathbb{B}(a, r), B_{0} \cap \mathbb{B}(b, r) ; D \cap \mathbb{B}(a, r), G \cap \mathbb{B}(b, r)\right), Z\right)
$$

which admits $\mathcal{A}$-limit $f$ on $\mathbb{X}\left(A_{0} \cap \mathbb{B}(a, r), B_{0} \cap \mathbb{B}(b, r) ; D \cap \mathbb{B}(a, r), G \cap \mathbb{B}(b, r)\right)$. $b \in \bar{B}_{0}$.

Fix $0<\delta_{0}<\frac{1}{2}$. Then it follows from (8.1) that for $0<r \leq r_{0}, a \in \bar{A}_{0}$,

$$
D_{a, \delta_{0}, r} \times G_{b, \delta_{0}, r} \subset \widehat{\mathbb{X}}\left(A_{0} \cap \mathbb{B}(a, r), B_{0} \cap \mathbb{B}(b, r) ; D \cap \mathbb{B}(a, r), G \cap \mathbb{B}(b, r)\right) .
$$

This, combined with (8.3), implies that

$$
\hat{f}_{a, b} \in \mathcal{O}\left(D_{a, \delta_{0}, r} \times G_{b, \delta_{0}, r}, Z\right), \quad 0<r \leq r_{0}, a \in \bar{A}_{0}, b \in \bar{B}_{0} .
$$

Next we fix a finite covering $\left(A_{0} \cap \mathbb{B}\left(a_{m}, r\right)\right)_{m=1}^{M}$ of $A_{0}$ and $\left(B_{0} \cap \mathbb{B}\left(b_{n}, r\right)\right)_{n=1}^{N}$ of $B_{0}$, where $\left(a_{m}\right)_{m=1}^{M} \subset \bar{A}_{0}$ and $\left(b_{n}\right)_{n=1}^{N} \subset \bar{B}_{0}$.

We divide the proof into two steps.

Step 1. Fix an open set $G^{\prime} \Subset G$. Then there exists $r_{1}: 0<r_{1}<r_{0}$ with the following property: for every $a \in \bar{A}_{0}$ there exist an open subset $A_{a}$ of $D$ and a mapping

$$
\hat{f}=\hat{f}_{a} \in \mathcal{O}\left(A_{a} \times\left(G^{\prime} \cup \bigcup_{n=1}^{N} G_{b_{n}, \delta_{0}, r_{0}}\right), Z\right)
$$


such that

$$
\hat{f}(z, w)=\hat{f}_{a, b_{n}}(z, w), \quad(z, w) \in\left(A_{a} \cap D_{a, \delta_{0}, r_{0}}\right) \times G_{b_{n}, \delta_{0}, r_{0}}, \quad n=1, \ldots, N ;
$$

and that $A_{a}$ is of the form $\left\{z \in D \cap \mathbb{B}\left(a, r_{1}\right): \omega\left(z, A_{0} \cap \mathbb{B}\left(a, r_{1}\right), D \cap \mathbb{B}\left(a, r_{1}\right)\right)<\right.$ $\delta_{a}$ \} for some $0<\delta_{a}<\delta_{0}$.

Proof of Step 1. Fix an arbitrary point $a_{0} \in \bar{A}_{0}$. First we claim that there are a sufficiently small number $r_{1}: 0<r_{1}<r_{0}$ and a finite number of open subsets $\left(V_{n}\right)_{n=1}^{N_{0}}$ of $G$ with the following properties:

(a) $V_{1}=G_{b_{1}, \delta_{0}, r_{0}}$ and $\left(G_{b_{n}, \delta_{0}, r_{0}}\right)_{n=1}^{N} \subset\left(V_{n}\right)_{n=1}^{N_{0}}$ (see the notation in (8.1));

(b) $\left.f\right|_{\left(\bar{A}_{0} \cap \mathbb{B}\left(a, r_{1}\right)\right) \times V_{n}}$ is bounded, $n=1, \ldots, N_{0}$;

(c) $G^{\prime} \Subset \bigcup_{n=1}^{N_{0}} V_{n}$;

(d) $V_{n} \cap V_{n+1} \neq \varnothing, n=1, \ldots, N_{0}-1$.

Indeed, we first start with the test $r_{1}:=r_{0}$ and $N_{0}:=N$ and $\left(V_{n}\right)_{n=1}^{N_{0}}:=\left(G_{b_{n}, \delta_{0}}\right)_{n=1}^{N}$. In virtue of (8.2) we see that our choice satisfies (a)-(b). If (c)-(d) are satisfied then we are done. Otherwise, we will make the following procedure.

Fix a point $w_{0} \in G^{\prime}$. For $n=1, \ldots, N$, let $\gamma_{n}:[0,1] \rightarrow G$ be a continuous one-to-one map such that

$$
\gamma_{n}(0)=w_{0} \quad \text { and } \quad \gamma_{n}(1) \in G_{b_{n}, \delta_{0}, r_{0}} .
$$

Since $f$ is locally bounded, there exist sufficiently small numbers $r_{1}, s: 0<r_{1} \leq$ $r_{0}$ and $0<s$ such that $\left.f\right|_{\left(\bar{A}_{0} \cap \mathbb{B}\left(a, r_{1}\right)\right) \times \mathbb{B}(w, s)}$ is bounded for all $a \in \bar{A}_{0}$ and $w \in$ $\overline{G^{\prime}} \bigcup_{n=1}^{N} \gamma_{n}([0,1])$. Therefore, we may add to the starting collection $\left(V_{n}\right)_{n=1}^{N}$ some balls of the form $\mathbb{B}(w, s)$, where $w \in \overline{G^{\prime}} \bigcup_{n=1}^{N} \gamma_{n}([0,1])$, and the new collection $\left(V_{n}\right)_{n=1}^{N_{0}}$ still satisfies (a)-(b). Now it remains to show that by adding a finite number of suitable balls $\mathbb{B}(w, s)$, (c)-(d) are also satisfied. But this assertion follows from an almost obvious geometric argument. In fact, we may renumber the collection $\left(V_{n}\right)$ if necessary. Hence, the above claim has been shown.

Using (c)-(d) above we may fix open sets $U_{n} \Subset V_{n}$ for $n=1, \ldots, N_{0}$, such that

$$
G^{\prime} \Subset \bigcup_{n=1}^{N_{0}} U_{n} \quad \text { and } \quad U_{n} \cap U_{n-1} \neq \varnothing, \quad 1<n \leq N_{0} .
$$

In what follows we will find the desired set $A_{a_{0}}$ and the desired holomorphic mapping $\hat{f}$ after $N_{0}$ steps. Namely, after the $n$-th step $\left(1 \leq n \leq N_{0}\right)$, we construct an open subset $A_{n}$ of $D$ in the form $D_{a_{0}, \delta_{n}, r_{1}}$ for a suitable $\delta_{n}>0$, and a mapping $\hat{f}_{n} \in \mathcal{O}\left(A_{n} \times\left(\bigcup_{p=1}^{n} U_{p}\right), Z\right)$. Finally, we obtain $A_{a_{0}}:=A_{N_{0}}$ and $\hat{f}:=\hat{f}_{N_{0}}$. Now we carry out this construction. 
In the first step, using (8.1), (8.3), (8.4) and (a), we define $\delta_{1}:=\delta_{0}, A_{1}:=D_{a_{0}, \delta_{1}, r_{1}} \quad$ and $\quad \hat{f}_{1}(z, w):=\hat{f}_{a_{0}, b_{1}}(z, w), \quad(z, w) \in A_{1} \times U_{1}$.

Suppose that we have constructed an open subset $A_{n-1}$ of $D$ and a mapping $\hat{f}_{n-1} \in$ $\mathcal{O}\left(A_{n-1} \times\left(\bigcup_{p=1}^{n-1} U_{p}\right), Z\right)$ for some $n: 2 \leq n \leq N_{0}$. We wish to construct an open subset $A_{n}$ of $D$ and a mapping $\hat{f}_{n} \in \mathcal{O}\left(A_{n} \times\left(\bigcup_{p=1}^{n} U_{p}\right), Z\right)$. There are two cases to consider.

Case. $V_{n}=G_{b_{m}, \delta_{0}}$ for some $1 \leq m \leq N$

In this case let $\delta_{n}:=\delta_{n-1}$ and $A_{n}:=A_{n-1}=D_{a_{0}, \delta_{n-1}, r_{1}}$, and

$$
\hat{f}_{n}:= \begin{cases}\hat{f}_{n-1}, & \text { on } A_{n} \times\left(\bigcup_{l=1}^{n-1} U_{l}\right), \\ \hat{f}_{a_{0}, b_{m}}, & \text { on } A_{n} \times U_{n} .\end{cases}
$$

Case. $V_{n} \notin\left(G_{b_{m}, \delta_{0}}\right)_{m=1}^{N}$

By (8.5) fix a nonempty open set $K \Subset U_{n} \cap U_{n-1}$. Then by the induction, $\hat{f}_{n-1} \in$ $\mathcal{O}\left(A_{n-1} \times K, Z\right)$. Recall from (b) that $f:\left(\bar{A}_{0} \cap \mathbb{B}\left(a_{0}, r_{1}\right)\right) \times V_{n} \longrightarrow Z$ is bounded. Since $f$ is locally bounded, by decreasing $r_{1}>0$ (if necessary) we may assume that

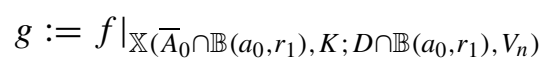

is bounded. Applying Theorem 7.4 to $g$, we obtain

$$
\hat{g} \in \mathcal{O}\left(\widehat{\mathbb{X}}\left(A_{0} \cap \mathbb{B}\left(a_{0}, r_{1}\right), K ; D \cap \mathbb{B}\left(a_{0}, r_{1}\right), V_{n}\right), Z\right)
$$

which extends $g$. Since $U_{n} \Subset V_{n}$, we may choose $\delta_{n}$ such that $0<\delta_{n}<1-$ $\sup _{w \in U_{n}} \omega\left(w, K, V_{n}\right)$. Using this and (8.1), it follows that

$$
D_{a_{0}, \delta_{n}, r_{1}} \times U_{n} \subset \widehat{\mathbb{X}}\left(A_{0} \cap \mathbb{B}\left(a_{0}, r_{1}\right), K ; D \cap \mathbb{B}\left(a_{0}, r_{1}\right), V_{n}\right) .
$$

Therefore, let $A_{n}:=D_{a_{0}, \delta_{n}, r_{1}}$ and define

$$
\hat{f}_{n}:= \begin{cases}\hat{f}_{n-1}, & \text { on } A_{n} \times\left(\bigcup_{l=1}^{n-1} U_{l}\right), \\ \hat{g}, & \text { on } A_{n} \times U_{n} .\end{cases}
$$

This completes our construction in the $n$-step. Finally, we put $A_{a_{0}}:=A_{N_{0}}$ and $\hat{f}_{a_{0}}:=\hat{f}_{N_{0}}$. Using this and (8.3) and (8.5) and (a), the desired conclusion of Step 1 follows.

Step 2. Completion of the proof. 
Proof of Step 2. Fix a sequence of relatively compact open subsets $\left(D_{k}^{\prime}\right)_{k=1}^{\infty}$ of $D$ (respectively $\left(G_{k}^{\prime}\right)_{k=1}^{\infty}$ of $G$ ) such that $D_{k}^{\prime} \nearrow D$ and $G_{k}^{\prime} \nearrow G$ as $k \nearrow \infty$. Put

$$
D_{k}:=D_{k}^{\prime} \cup \bigcup_{m=1}^{M} D_{a_{m}, \delta_{0}, r_{0}}, \quad G_{k}:=G_{k}^{\prime} \cup \bigcup_{n=1}^{N} G_{b_{n}, \delta_{0}, r_{0}}, \quad k \geq 1 .
$$

Using the result of Step 1, we may find, for every $k$, a number $0<r_{k}<r_{0}$ with the following properties:

- for every $a \in A_{0}$, there is $0<\delta_{a, k}<\delta_{0}$ such that by considering the open set

$$
A_{a, k}:=\left\{z \in D \cap \mathbb{B}\left(a, r_{k}\right): \omega\left(z, A_{0} \cap \mathbb{B}\left(a, r_{k}\right), D \cap \mathbb{B}\left(a, r_{k}\right)\right)<\delta_{a, k}\right\}
$$

one can find a mapping $\hat{f}_{a, k} \in \mathcal{O}\left(A_{a, k} \times G_{k}, Z\right)$ satisfying

$$
\hat{f}_{a, k}=\hat{f}_{a, b_{n}} \quad \text { on }\left(A_{a, k} \cap D_{a, \delta_{0}, r_{k}}\right) \times G_{b_{n}, \delta_{0}, r_{k}}, \quad n=1, \ldots, N ;
$$

- for every $b \in B$, there is $0<\delta_{b, k}<\delta_{0}$ such that by considering the open set

$$
B_{b, k}:=\left\{w \in G \cap \mathbb{B}\left(b, r_{k}\right): \omega\left(z, B_{0} \cap \mathbb{B}\left(b, r_{k}\right), G \cap \mathbb{B}\left(b, r_{k}\right)\right)<\delta_{b, k}\right\}
$$

one can find a mapping $\hat{f}_{b, k} \in \mathcal{O}\left(D_{k} \times B_{b, k}, Z\right)$ satisfying

$$
\hat{f}_{b, k}=\hat{f}_{a_{m}, b} \quad \text { on } D_{a_{m}, \delta_{0}, r_{k}} \times\left(B_{b, k} \cap G_{b, \delta_{0}, r_{k}}\right), \quad m=1, \ldots, M .
$$

Next using the compactness of $\bar{A}_{0}$ and $\bar{B}_{0}$, one may find, for every $k$, two finite coverings $\left(A_{0} \cap \mathbb{B}\left(a_{m}^{\prime}, r_{k}\right)\right)_{m^{\prime}=1}^{M_{k}}$ of $A_{0}$ and $\left(B_{0} \cap \mathbb{B}\left(b_{n^{\prime}}, r_{k}\right)\right)_{n^{\prime}=1}^{N_{k}}$ of $B_{0}$, where $\left(a_{m^{\prime}}\right)_{m^{\prime}=1}^{M_{k}} \subset \bar{A}_{0}$ and $\left(b_{n^{\prime}}\right)_{n^{\prime}=1}^{N_{k}} \subset \bar{B}_{0}$. Put

$$
A_{k}:=\bigcup_{m^{\prime}=1}^{M_{k}} A_{a_{m^{\prime}}, k} \quad \text { and } \quad B_{k}:=\bigcup_{n^{\prime}=1}^{N_{k}} B_{b_{n^{\prime}}, k}, \quad k \geq 1 .
$$

In virtue of (8.6)-(8.9) and (8.2)-(8.4), the family $\left(\hat{f}_{a_{m^{\prime}}, k}\right)_{m^{\prime}=1}^{M_{k}} \cup\left(\hat{f}_{b_{n^{\prime}, k}}\right)_{n^{\prime}=1}^{N_{k}}$ is collective for every $k \geq 1$. Let

$$
\hat{f}_{k} \in \mathcal{O}\left(\mathbb{X}\left(A_{k}, B_{k} ; D_{k}, G_{k}\right), Z\right)
$$

denote the collected mapping of this family.

Next, we show that

$$
\begin{aligned}
& \lim _{k \rightarrow \infty} \omega\left(z, A_{0}, D_{k}\right)=\omega\left(z, A_{0}, D\right) \text { and } \\
& \lim _{k \rightarrow \infty} \omega\left(w, B_{0}, G_{k}\right)=\omega\left(z, B_{0}, G\right), \quad z \in D, w \in G .
\end{aligned}
$$


It is sufficient to prove the first identity in (8.11) since the proof of the second one is similar. Observe that there is $u \in \mathcal{P} \mathcal{S H}(D)$ such that $\omega\left(\cdot, A_{0}, D_{k}\right) \searrow u$ as $k \nearrow \infty$ and $u \geq \omega\left(\cdot, A_{0}, D\right)$ on $D$. Therefore, the proof of (8.11) will be complete if one can show that $u \leq \omega\left(\cdot, A_{0}, D\right)$ on $D$.

To this end observe that for every $a \in A_{0}$ there is $1 \leq m \leq M$ such that $a \in \mathbb{B}\left(a_{m}, r_{0}\right)$. Consequently, using (8.6),

$$
(\mathcal{A}-\lim \sup u)(a) \leq\left(\mathcal{A}-\lim \sup \omega\left(\cdot, A_{0} \cap \mathbb{B}\left(a_{m}, r_{0}\right), D_{a_{m}, \delta_{0}, r_{0}}\right)\right)(a)=0
$$

where the equality follows from an application of Proposition 3.5. This, combined with the obvious inequality $u \leq 1$, implies that $u \leq \omega\left(\cdot, A_{0}, D\right)$. Hence, (8.11) follows.

We are now in the position to define the desired extension mapping $\hat{f}$. Indeed, one glues $\left(\hat{f}_{k}\right)_{k=1}^{\infty}$ given in (8.10) together to obtain $\hat{f}$ in the following way

$$
\hat{f}:=\lim _{k \rightarrow \infty} \hat{f}_{k} \quad \text { on } \widehat{W}_{0}
$$

One needs to check that the last limit exists and possesses all the required properties. In virtue of (8.7)-(8.11), and the Gluing Lemma 7.6, the proof will be complete if we can show the following

Claim. For every $\left(z_{0}, w_{0}\right) \in \widehat{W}_{0}$, there are an open neighborhood $U \times V$ of $\left(z_{0}, w_{0}\right)$ and $\delta_{0}>0$ such that the hypotheses of Lemma 7.6 is fulfilled with

$$
\mathcal{D}:=D, \mathcal{G}:=G, \mathcal{P}_{k}:=A_{k}, \mathcal{Q}_{k}:=B_{k}, \mathcal{D}_{k}:=D_{k}, \mathcal{G}_{k}:=G_{k}, \quad k \geq 1
$$

To this end let

$$
\delta_{0}:=\frac{1-\omega\left(z_{0}, A_{0}, D\right)-\omega\left(w_{0}, B_{0}, G\right)}{2},
$$

and let $U \times V$ be an open neighborhood of $\left(z_{0}, w_{0}\right)$ such that

$$
\omega\left(z, A_{0}, D\right)+\omega\left(w, B_{0}, G\right)<\omega\left(z_{0}, A_{0}, D\right)+\omega\left(w_{0}, B_{0}, G\right)+\delta_{0}
$$

Then using these inequalities and (8.11), we see that there is a sufficiently big $q_{0} \in$ $\mathbb{N}$ such that for $q_{0} \leq q \leq p$ and $(z, w) \in U \times V$,

$$
\begin{aligned}
\omega\left(z, A_{p}, D_{q}\right)+\omega\left(w, B_{p}, D_{q}\right) & \leq \omega\left(z, A_{0}, D_{q}\right)+\omega\left(w, B_{0}, G_{q}\right) \\
& \leq \omega\left(z, A_{0}, D\right)+\omega\left(w, B_{0}, G\right)+\delta_{0}<1
\end{aligned}
$$

This proves the above claim. Hence, the proof of the theorem is finished.

Now we are able to formulate the following semi-local result. 
Theorem 8.3. Let $D$ be an open subset of a complex manifold and $G \subset \mathbb{C}^{m} a$ bounded open set and $Z$ a complex analytic space possessing the Hartogs extension property. $D$ (respectively $G$ ) is equipped with the canonical system of approach regions (respectively the system of approach regions $\left.\left(\mathcal{A}_{\beta}(\eta)\right)_{\eta \in \bar{G}, \alpha \in I_{\eta}}\right)$. Let $A$ be an open subset of $D$ and let $B, B_{0}$ be subsets of $\bar{G}$ such that $B_{0}$ is locally pluriregular and $\bar{B}_{0} \subset B^{*}$. Put

$$
W:=\mathbb{X}(A, B ; D, G) \quad \text { and } \quad W_{0}:=\mathbb{X}\left(A, B_{0} ; D, G\right) .
$$

Then, for every mapping $f: W \longrightarrow Z$ which satisfies the following conditions:

- $f \in \mathcal{C}_{s}(W, Z) \cap \mathcal{O}_{s}\left(W^{o}, Z\right)$;

- $f$ is locally bounded along $D \times(B \cap \partial G)$,

there exists a unique mapping $\hat{f} \in \mathcal{O}\left(\widehat{W}_{0}, Z\right)$ which admits $\mathcal{A}$-limit $f(\zeta, \eta)$ at all points $(\zeta, \eta) \in W_{0}$.

Proof. First, applying Part 1) of Theorem 7.2 and using the hypotheses on $f$, we see that $f$ extends to a mapping (still denoted by) $f$ defined on $\mathbb{X}\left(A, B \cup B^{*} ; D, G\right)$ such that $f$ is separately holomorphic on $\mathbb{X}^{\circ}\left(A, B \cup B^{*} ; D, G\right)$ and that $\left.f\right|_{\mathbb{X}\left(A, B^{*} ; D, G\right)}$ is locally bounded.

We define $\hat{f}$ at a point $\left(z_{0}, w_{0}\right) \in \widehat{W}_{0}$ as follows: Let $\epsilon>0$ be such that

$$
\omega\left(z_{0}, A, D\right)+\omega\left(w_{0}, B_{0}, G\right)+\epsilon<1 .
$$

By Theorem 3.1 and Proposition 3.4, there is a holomorphic disc $\phi \in \mathcal{O}(\bar{E}, D)$ such that $\phi(0)=z_{0}$ and

$$
1-\frac{1}{2 \pi} \cdot \operatorname{mes}\left(\phi^{-1}(A) \cap \partial E\right)<\omega\left(z_{0}, A, D\right)+\epsilon .
$$

Moreover, using the hypotheses, we see that the mapping $f_{\phi}$, defined by

$$
f_{\phi}(t, w):=f(\phi(t), w), \quad(t, w) \in \mathbb{X}\left(\phi^{-1}(A) \cap \partial E, B ; E, G\right),
$$

satisfies the hypotheses of Theorem 8.2. By this theorem, let $\hat{f}_{\phi}$ be the unique mapping in $\widehat{\mathbb{X}}\left(\phi^{-1}(A) \cap \partial E, B_{0} ; E, G\right)$ such that

$$
\left(\mathcal{A}-\lim \hat{f}_{\phi}\right)(t, w)=f_{\phi}(t, w), \quad(t, w) \in \mathbb{X}\left(\phi^{-1}(A) \cap \partial E, B_{0} ; E, G\right) .
$$

In virtue of (8.12)-(8.13), $\left(0, w_{0}\right) \in \widehat{\mathbb{X}}\left(\phi^{-1}(A) \cap \partial E, B_{0} ; E, G\right)$. Then the value at $\left(z_{0}, w_{0}\right)$ of the desired extension mapping $\hat{f}$ is given by

$$
\hat{f}\left(z_{0}, w_{0}\right):=\hat{f}_{\phi}\left(0, w_{0}\right) .
$$


Using this and (8.14)-(8.15), and arguing as in Part 2) of Lemma 4.5, one can show that $\hat{f}$ is well-defined on $\widehat{W}_{0}$.

To show that $\hat{f}$ is holomorphic, one argues as in Step 1 of the proof of Theorem 6.1. To show that $\hat{f}$ admits $\mathcal{A}$-limit $f(\zeta, \eta)$ at all points $(\zeta, \eta) \in W_{0}$ and that it is uniquely defined, one proceeds as in Step 2-4 of the proof of Theorem 6.1 making the obviously necessary changes and adaptations. Hence, the proof is finished.

\section{Proof of Theorem A}

First we need a variant of Definition 2.3. For a set $A \subset \bar{D}$, Let $\widetilde{\mathcal{E}}(A)$ be the set of all elements $P \in \mathcal{E}(A)$ with the property that there is an open neighborhood $U \subset X$ of $P$ such that $U$ is biholomorphic to a domain in some $\mathbb{C}^{n}$. Then it can be checked that

$$
\widetilde{A}:=\bigcup_{P \in \widetilde{\mathcal{E}}(A)} P .
$$

This identity will allow us to pass from "local informations" to "global extensions".

For the proof we need to develop some preparatory results.

In virtue of $(9.1)$, for any $P \in \widetilde{\mathcal{E}}(A)$ (respectively $Q \in \widetilde{\mathcal{E}}(B)$ ) fix an open neighborhood $U_{P}$ of $P$ (respectively $V_{Q}$ of $Q$ ) such that $U_{P}$ (respectively $V_{Q}$ ) is biholomorphic to a domain in $\mathbb{C}^{d_{P}}$ (respectively in $\mathbb{C}^{d_{Q}}$ ), where $d_{P}$ (respectively $d_{Q}$ ) is the dimension of $D$ (respectively $G$ ) at points of $P$ (respectively $Q$ ). For any $0<\delta \leq \frac{1}{2}$ define

$$
\begin{aligned}
U_{P, \delta} & :=\left\{z \in U_{P}: \omega\left(z, P, U_{P}\right)<\delta\right\}, \quad P \in \widetilde{\mathcal{E}}(A), \\
V_{Q, \delta} & :=\left\{w \in V_{Q}: \omega\left(w, Q, V_{Q}\right)<\delta\right\}, \quad Q \in \widetilde{\mathcal{E}}(B), \\
A_{\delta} & :=\bigcup_{P \in \widetilde{\mathcal{E}}(A)} U_{P, \delta}, \quad B_{\delta}:=\bigcup_{Q \in \widetilde{\mathcal{E}}(B)} V_{Q, \delta}, \\
D_{\delta} & :=\{z \in D: \widetilde{\omega}(z, A, D)<1-\delta\}, \\
G_{\delta} & :=\{w \in G: \widetilde{\omega}(w, B, G)<1-\delta\} .
\end{aligned}
$$

Lemma 9.1. We keep the above notation. Then:

(1) For every $\zeta \in \widetilde{A}$ and $\alpha \in I_{\zeta}$, there is an open neighborhood $\mathcal{U}$ of $\zeta$ such that $\mathcal{U} \cap \mathcal{A}_{\alpha}(\zeta) \subset A_{\delta}$

(2) $A_{\delta}$ is an open subset of $D$ and $A_{\delta} \subset D_{1-\delta} \subset D_{\delta}$.

(3) $\widetilde{\omega}(z, A, D)-\delta \leq \omega\left(z, A_{\delta}, D\right) \leq \widetilde{\omega}(z, A, D), z \in D$.

Proof of Lemma 9.1. To prove Part (1) fix, in view of (9.1)-(9.2), $P \in \widetilde{\mathcal{E}}(A)$, $\zeta \in P$ and $\alpha \in I_{\zeta}$. Using the definition of local pluriregularity, we see that $\limsup \operatorname{su}_{z \rightarrow \zeta, z \in \mathcal{A}_{\alpha}(\zeta)} \omega\left(z, P, U_{P}\right)=0$. Hence, Part (1) follows.

The assertion that $A_{\delta}$ is open follows immediately from (9.2). Since $0<\delta \leq$ $\frac{1}{2}$, the second inclusion in Part (2) is clear. To prove the first inclusion let $z$ be an 
arbitrary point of $A_{\delta}$. Then there is $P \in \widetilde{\mathcal{E}}(A)$ such that $z \in U_{P, \delta}$. Using (9.2) and Definition 2.3 we obtain

$$
\widetilde{\omega}(z, A, D)=\omega(z, \widetilde{A}, D) \leq \omega\left(z, P, U_{P}\right)<\delta .
$$

Hence, $z \in D_{1-\delta}$, which in turn implies that $A_{\delta} \subset D_{1-\delta}$.

It follows from Part (1) that

$$
\omega\left(z, A_{\delta}, D\right) \leq \omega(z, \widetilde{A}, D)=\widetilde{\omega}(z, A, D), \quad z \in D,
$$

which proves the second estimate in Part (3). To complete the proof let $P \in \widetilde{\mathcal{E}}(A)$ and $0<\delta \leq \frac{1}{2}$. We deduce from (9.3) that $\widetilde{\omega}(z, A, D)-\delta \leq 0$ for $z \in U_{P, \delta}$. Hence, by $(9.2)$,

$$
\widetilde{\omega}(z, A, D)-\delta \leq 0, \quad z \in A_{\delta} .
$$

On the other hand, $\widetilde{\omega}(z, A, D)-\delta<1, z \in D$. Recall from Part (2) that $A_{\delta}$ is an open subset of $D_{\delta}$. Consequently, the first estimate of Part (3) follows.

Now we are able to to prove Theorem A in the following special case.

Proposition 9.2. Let $D$ be an open subset of a complex manifold and $G$ a bounded open subset of $\mathbb{C}^{m}$ and $Z$ a complex analytic space possessing the Hartogs extension property. $D$ (respectively $G$ ) is equipped with a system of approach regions $\left(\mathcal{A}_{\alpha}(\zeta)\right)_{\zeta \in \bar{D}, \alpha \in I_{\zeta}}$ (respectively $\left(\mathcal{A}_{\beta}(\eta)\right)_{\eta \in \bar{G}, \beta \in I_{\eta}}$ ). Let $A$ be a subset of $\bar{D}$, let $B$, $B_{0}$ be subsets of $\bar{G}$ such that $B_{0}$ is locally pluriregular and $\bar{B}_{0} \subset B^{*}$. Put

$$
\begin{aligned}
W & :=\mathbb{X}(A, B ; D, G), \quad W_{0}:=\mathbb{X}\left(A, B_{0} ; D, G\right), \\
\widetilde{W}^{o} & :=\left((D \cup \widetilde{A}) \times B_{0}\right) \bigcup\left(\widetilde{A} \times\left(G \cup B_{0}\right)\right), \\
\widehat{W}^{o} & :=\left\{(z, w) \in D \times G: \widetilde{\omega}(z, A, D)+\omega\left(w, B_{0}, G\right)<1\right\} .
\end{aligned}
$$

Then, for every mapping $f: W \longrightarrow Z$ which satisfies the following conditions:

- $f \in \mathcal{C}_{s}(W, Z) \cap \mathcal{O}_{s}\left(W^{o}, Z\right)$;

- $f$ is locally bounded along $\mathbb{X}(A \cap \partial D, B \cap \partial G ; D, G)$;

- $\left.f\right|_{A \times B}$ is continuous at all points of $(A \cap \partial D) \times(B \cap \partial G)$,

there exists a unique mapping $\hat{f} \in \mathcal{O}\left(\widehat{W}^{o}, Z\right)$ which admits $\mathcal{A}$-limit $f(\zeta, \eta)$ at all points $(\zeta, \eta) \in \widetilde{W}^{o}$.

Proof of Proposition 9.2. First, applying Part 1) of Theorem 7.2 and using the hypotheses on $f$, we see that $f$ extends to a mapping (still denoted by $f$ ) defined on $\mathbb{X}\left(A \cup A^{*}, B \cup B^{*} ; D, G\right)$ such that $f$ is separately holomorphic on $\mathbb{X}^{\circ}\left(A \cup A^{*}, B \cup\right.$ $\left.B^{*} ; D, G\right)$ and that $\left.f\right|_{\mathbb{X}\left(A^{*}, B^{*} ; D, G\right)}$ is locally bounded.

For each $P \in \widetilde{\mathcal{E}}(A), U_{P}$ (respectively $G$ ) is biholomorphic to an open set in $\mathbb{C}^{d_{P}}$ (respectively in $\left.\mathbb{C}^{m}\right)$. Consequently, the mapping $f_{P}:=\left.f\right|_{\mathbb{X}\left(\bar{P}, B ; U_{P}, G\right)}$ 
satisfies the hypotheses of Theorem 8.2. Hence, we obtain a unique mapping $\hat{f}_{P} \in$ $\mathcal{O}\left(\widehat{\mathbb{X}}\left(P, B_{0} ; U_{P}, G\right), Z\right)$ such that

$$
\left(\mathcal{A}-\lim \hat{f}_{P}\right)(z, w)=f_{P}(z, w)=f(z, w), \quad(z, w) \in \mathbb{X}\left(P, B_{0} ; U_{P}, G\right) .
$$

Let $0<\delta \leq \frac{1}{2}$ and $G_{\delta}^{\prime}:=\left\{w \in G: \omega\left(w, B_{0}, G\right)<1-\delta\right\}$. We will show that the family $\left(\left.\hat{f}_{P}\right|_{U_{P, \delta} \times G_{\delta}^{\prime}}\right)_{P \in \widetilde{\mathcal{E}}(A)}$ is collective in the sense of Definition 8.1, where $U_{P, \delta}$ is given in (9.2).

To prove this assertion let $P_{1}, P_{2}$ be arbitrary elements of $\widetilde{\mathcal{E}}(A)$. By (9.4), we have

$$
\begin{aligned}
\left(\mathcal{A}-\lim \hat{f}_{P_{1}}\right)(z, w) & =f(z, w)=\left(\mathcal{A}-\lim \hat{f}_{P_{2}}\right)(z, w), \\
(z, w) & \in\left(U_{P_{1}} \cap U_{P_{2}}\right) \times B_{0} .
\end{aligned}
$$

The assertion is reduced to showing that

$$
\begin{aligned}
\hat{f}_{P_{1}}(z, w) & =\hat{f}_{P_{2}}(z, w), \\
(z, w) & \in \widehat{\mathbb{X}}\left(P_{1}, B_{0} ; U_{P_{1}}, G\right) \cap \widehat{\mathbb{X}}\left(P_{2}, B_{0} ; U_{P_{2}}, G\right) .
\end{aligned}
$$

To this end fix $\left(z_{0}, w_{0}\right) \in \widehat{\mathbb{X}}\left(P_{1}, B_{0} ; U_{P_{1}}, G\right) \cap \widehat{\mathbb{X}}\left(P_{2}, B_{0} ; U_{P_{2}}, G\right)$. Observe that both mappings $w \mapsto \hat{f}_{P_{1}}\left(z_{0}, w\right)$ and $w \mapsto \hat{f}_{P_{2}}\left(z_{0}, w\right)$ belong to $\mathcal{O}(\mathcal{G}, Z)$, where $\mathcal{G}$ is the connected component which contains $w_{0}$ of the following open set

$$
\left\{w \in G: \omega\left(w, B_{0}, G\right)<1-\max _{j \in\{1,2\}} \omega\left(z_{0}, P_{j}, U_{j}\right)\right\} .
$$

Applying Theorem 7.5 to these mappings using (9.5), Proposition 3.5 and (9.6), the above assertion follows.

In virtue of (9.2) let

$$
\tilde{\tilde{f}}_{\delta} \in \mathcal{O}\left(A_{\delta} \times G_{\delta}^{\prime}, Z\right)
$$

denote the collected mapping of the family $\left(\left.\hat{f}_{P}\right|_{U_{P, \delta} \times G_{\delta}^{\prime}}\right)_{P \in \widetilde{\mathcal{E}}(A)}$. In virtue of (9.4) and (9.7), we are able to define a new mapping $\tilde{f}_{\delta}$ on $\mathbb{X}\left(A_{\delta}, B ; D, G_{\delta}^{\prime}\right)$ as follows

$$
\tilde{f}_{\delta}:= \begin{cases}\tilde{\tilde{f}}, & \text { on } A_{\delta} \times G_{\delta}^{\prime}, \\ f, & \text { on } D \times B .\end{cases}
$$

Using this and (9.4)-(9.7), we see that

$$
\mathcal{A}-\lim \tilde{f}_{\delta}=f \quad \text { on } \quad \mathbb{X}\left(A \cap \tilde{A}, B_{0} ; D, G_{\delta}^{\prime}\right) .
$$


Since $A_{\delta}$ is an open subset of $X$ and $G_{\delta}^{\prime}$ is a bounded open set in $\mathbb{C}^{m}$, we are able to apply Theorem 8.3 to $\tilde{f}_{\delta}$ in order to obtain a mapping $\hat{f}_{\delta} \in \mathcal{O}\left(\widehat{\mathbb{X}}\left(A_{\delta}, B_{0} ; D, G_{\delta}^{\prime}\right), Z\right)$ such that

$$
\mathcal{A}-\lim \hat{f}_{\delta}=\tilde{f}_{\delta} \quad \text { on } \quad \mathbb{X}\left(A_{\delta}, B_{0} ; D, G_{\delta}^{\prime}\right) .
$$

We are now in a position to define the desired extension mapping $\hat{f}$. Indeed, one glues $\left(\hat{f}_{\delta}\right)_{0<\delta \leq \frac{1}{2}}$ together to obtain $\hat{f}$ in the following way

$$
\hat{f}:=\lim _{k \rightarrow \infty} \hat{f}_{\frac{1}{k}} \quad \text { on } \widehat{W}^{\mathrm{o}} .
$$

One needs to check that the last limit exists and possesses all the required properties. In virtue of (9.8)-(9.9) and Lemma 7.6, the proof will be complete if one can show that for every $\left(z_{0}, w_{0}\right) \in \widehat{W}^{\mathrm{o}}$, there are an open neighborhood $U \times V$ of $\left(z_{0}, w_{0}\right)$ and $\delta_{0}>0$ such that hypothesis (iii) of Lemma 7.6 is fulfilled with

$$
\mathcal{D}:=D, \mathcal{G}:=G, \mathcal{P}_{k}:=A_{\frac{1}{k}}, \mathcal{Q}_{k}:=B_{0}, \mathcal{D}_{k}:=D, \mathcal{G}_{k}:=G_{\frac{1}{k}}^{\prime}, \quad k>2 .
$$

To this end let

$$
\delta_{0}:=\frac{1-\widetilde{\omega}\left(z_{0}, A, D\right)-\omega\left(w_{0}, B_{0}, G\right)}{2},
$$

and let $U \times V$ be an open neighborhood of $\left(z_{0}, w_{0}\right)$ such that

$$
\widetilde{\omega}(z, A, D)+\omega\left(w, B_{0}, G\right)<\widetilde{\omega}\left(z_{0}, A, D\right)+\omega\left(w_{0}, B_{0}, G\right)+\delta_{0} .
$$

Then for $k>\frac{1}{\delta_{0}}$ and for $(z, w) \in U \times V$, using the last inequality, and applying Part (3) of Lemma 9.1 and Proposition 3.5, we see that

$$
\begin{aligned}
\widetilde{\omega}\left(z, A_{\frac{1}{k}}, D\right)+\omega\left(w, B_{0}, G_{\delta_{0}}^{\prime}\right) & \leq \widetilde{\omega}(z, A, D)+\frac{\omega\left(w, B_{0}, G\right)}{1-\delta_{0}} \\
& \leq \frac{\widetilde{\omega}(z, A, D)+\omega\left(w, B_{0}, G\right)}{1-\delta_{0}}<1 .
\end{aligned}
$$

This proves the above assertion. Hence, the proof of the proposition is finished.

We now arrive at

Proof of Theorem A. First, applying Part 1) of Theorem 7.2 and using the hypotheses on $f$, we see that $f$ extends to a mapping (still denoted by) $f$ defined on $\mathbb{X}\left(A \cup A^{*}, B \cup B^{*} ; D, G\right)$ such that $f$ is separately holomorphic on $\mathbb{X}^{\mathrm{o}}\left(A \cup A^{*}, B \cup\right.$ $\left.B^{*} ; D, G\right)$ and that $\left.f\right|_{\mathbb{X}\left(A^{*}, B^{*} ; D, G\right)}$ is locally bounded. 
For each $P \in \widetilde{\mathcal{E}}(A), U_{P}$ is biholomorphic to an open set in $\mathbb{C}^{d_{P}}$. Consequently, the mapping $f_{P}:=\left.f\right|_{\mathbb{X}\left(\bar{P}, B ; U_{P}, G\right)}$ satisfies the hypotheses of Proposition 9.2. Hence, we obtain a unique mapping $\hat{f}_{P} \in \mathcal{O}\left(\widehat{\mathbb{X}}^{\circ}\left(P, B ; U_{P}, G\right), Z\right)^{11}$ such that

$$
\left(\mathcal{A}-\lim \hat{f}_{P}\right)(z, w)=f(z, w), \quad(z, w) \in \mathbb{X}\left(P, \widetilde{B} \cap B ; U_{P}, G\right) .
$$

Let $0<\delta \leq \frac{1}{2}$. Using (9.10) and arguing as in the proof of Proposition 9.2, we may collect the family $\left(\left.\hat{f}_{P}\right|_{U_{P, \delta} \times G_{\delta}}\right)_{P \in \widetilde{\mathcal{E}}(A)}$ in order to obtain the collected mapping $\tilde{f}_{\delta}^{A} \in \mathcal{O}\left(A_{\delta} \times G_{\delta}, Z\right)$.

Similarly, for each $Q \in \widetilde{\mathcal{E}}(B)$, one obtains a unique mapping $\hat{f}_{Q} \in$ $\mathcal{O}\left(\widehat{\mathbb{X}}^{\mathrm{o}}\left(A, Q ; D, V_{Q}\right), Z\right)^{12}$ such that

$$
\left(\mathcal{A}-\lim \hat{f}_{Q}\right)(z, w)=f(z, w), \quad(z, w) \in \mathbb{X}\left(A \cap \widetilde{A}, Q ; D, V_{Q}\right) .
$$

Moreover, one can collect the family $\left(\left.\hat{f}_{Q}\right|_{D_{\delta} \times V_{Q, \delta}}\right)_{Q \in \widetilde{\mathcal{E}}(B)}$ in order to obtain the collected mapping $\tilde{f}_{\delta}^{B} \in \mathcal{O}\left(D_{\delta} \times B_{\delta}, Z\right)$.

Next, we prove that

$$
\tilde{f}_{\delta}^{A}=\tilde{f}_{\delta}^{B} \quad \text { on } A_{\delta} \times B_{\delta} .
$$

Indeed, in virtue of (9.10)-(9.11) it suffices to show that for any $P \in \widetilde{\mathcal{E}}(A)$ and $Q \in \widetilde{\mathcal{E}}(B)$ and any $0<\delta \leq \frac{1}{2}$,

$$
\hat{f}_{P}(z, w)=\hat{f}_{Q}(z, w), \quad(z, w) \in U_{P, \delta} \times V_{Q, \delta} .
$$

Observe that in virtue of (9.10)-(9.11) one has that

$$
\left(\mathcal{A}-\lim \hat{f}_{P}\right)(z, w)=\left(\mathcal{A}-\lim \hat{f}_{Q}\right)(z, w)=f(z, w), \quad(z, w) \in \mathbb{X}\left(P, Q ; U_{P}, V_{Q}\right) .
$$

Recall that $U_{P}$ (respectively $V_{Q}$ ) is biholomorphic to a domain in $\mathbb{C}^{d_{P}}$ (respectively $\mathbb{C}^{d_{Q}}$ ). Consequently, applying the uniqueness of Theorem 8.2 yields that

$$
\hat{f}_{P}(z, w)=\hat{f}_{Q}(z, w), \quad(z, w) \in \widehat{\mathbb{X}}\left(P, Q ; U_{P}, V_{Q}\right) .
$$

Hence, the proof of (9.13) and then the proof of (9.12) are finished.

In virtue of (9.12), we are able to define a new mapping $\tilde{f}_{\delta}: \mathbb{X}^{0}\left(A_{\delta}, B_{\delta}\right.$; $\left.D_{\delta}, G_{\delta}\right) \longrightarrow Z$ as follows

$$
\tilde{f}_{\delta}:= \begin{cases}\tilde{f}_{\delta}^{A}, & \text { on } A_{\delta} \times G_{\delta}, \\ \tilde{f}_{\delta}^{B}, & \text { on } D_{\delta} \times B_{\delta} .\end{cases}
$$

${ }^{11}$ Here $\widehat{\mathbb{X}}^{\mathrm{o}}\left(P, B ; U_{P}, G\right):=\left\{(z, w) \in U_{P} \times G: \omega\left(z, P, U_{P}\right)+\widetilde{\omega}(w, B, G)<1\right\}$.

12 Here $\widehat{\mathbb{X}}^{\mathrm{o}}\left(A, Q ; D, V_{Q}\right):=\left\{(z, w) \in D \times V_{Q}: \widetilde{\omega}(z, A, D)+\omega\left(w, Q, V_{Q}\right)<1\right\}$. 
Using formula (9.14) it can be readily checked that $\tilde{f}_{\delta} \in \mathcal{O}_{s}\left(\mathbb{X}^{\mathrm{o}}\left(A_{\delta}, B_{\delta} ; D_{\delta}, G_{\delta}\right), Z\right)$. Since we know from Part (2) of Lemma 9.1 that $A_{\delta}$ (respectively $B_{\delta}$ ) is an open subset of $D_{\delta}$ (respectively $G_{\delta}$ ), we are able to apply Theorem 7.3 to $\tilde{f}_{\delta}$ for every $0<$ $\delta \leq \frac{1}{2}$. Consequently, one obtains a unique mapping $\hat{f}_{\delta} \in \mathcal{O}\left(\widehat{\mathbb{X}}\left(A_{\delta}, B_{\delta} ; D_{\delta}, G_{\delta}\right), Z\right)$ such that

$$
\hat{f}_{\delta}=\tilde{f}_{\delta} \quad \text { on } \mathbb{X}^{\mathrm{o}}\left(A_{\delta}, B_{\delta} ; D_{\delta}, G_{\delta}\right) .
$$

It follows from (9.10)-(9.11) and (9.14)-(9.15) that

$$
\mathcal{A}-\lim \hat{f_{\delta}}=f \quad \text { on } \mathbb{X}\left(A \cap \widetilde{A}, B \cap \widetilde{B} ; D_{\delta}, G_{\delta}\right) .
$$

In addition, for any $0<\delta \leq \delta_{0} \leq \frac{1}{2}$, and any $(z, w) \in A_{\delta} \times B_{\delta}$, there is $P \in \widetilde{\mathcal{E}}(A)$ such that $z \in U_{P, \delta_{0}}$. Therefore, it follows from the construction of $\tilde{f}_{\delta}^{A},(9.14)$ and (9.15) that

$$
\hat{f}_{\delta}(z, w)=\hat{f}_{P}(z, w)=\hat{f}_{\delta_{0}}(z, w) .
$$

This proves that $\hat{f}_{\delta}=\hat{f}_{\delta_{0}}$ on $A_{\delta} \times B_{\delta}$ for $0<\delta \leq \delta_{0} \leq \frac{1}{2}$. Hence,

$$
\hat{f}_{\delta}=\hat{f}_{\delta_{0}} \quad \text { on } \mathbb{X}\left(A_{\delta}, B_{\delta} ; D_{\delta_{0}}, G_{\delta_{0}}\right), 0<\delta \leq \delta_{0} \leq \frac{1}{2} .
$$

We are now in a position to define the desired extension mapping $\hat{f}$.

$$
\hat{f}:=\lim _{k \rightarrow \infty} \hat{f}_{\frac{1}{k}} \quad \text { on } \widehat{\widetilde{W}} .
$$

To prove that $\hat{f}$ satisfies the desired conclusion of the theorem one proceeds as in the end of the proof of Proposition 9.2. In virtue of (9.16)-(9.17) and Lemma 7.6, the proof will be complete if we can verify that for every $\left(z_{0}, w_{0}\right) \in \widehat{W}$, there are an open neighborhood $U \times V$ of $\left(z_{0}, w_{0}\right)$ and $\delta_{0}>0$ such that hypothesis (iii) of Lemma 7.6 is fulfilled with

$$
\mathcal{D}:=D, \mathcal{G}:=G, \mathcal{P}_{k}:=A_{\frac{1}{k}}, \mathcal{Q}_{k}:=B_{\frac{1}{k}}, \mathcal{D}_{k}:=D_{\frac{1}{k}}, \mathcal{G}_{k}:=G_{\frac{1}{k}}, \quad k>2
$$

Since the verification follows along almost the same lines as that of Proposition 9.2, it is, therefore, left to the interested reader.

Hence, the proof of Theorem A is finished.

\section{Applications}

In this section we give various applications of Theorem A using different systems of approach regions defined in Subsection 2.2. 


\subsection{Canonical system of approach regions}

For every open subset $U \subset \mathbb{R}^{2 n-1}$ and every continuous function $h: U \longrightarrow \mathbb{R}$, the graph

$$
\left\{z=\left(z^{\prime}, z_{n}\right)=\left(z^{\prime}, x_{n}+i y_{n}\right) \in \mathbb{C}^{n}:\left(z^{\prime}, x_{n}\right) \in U \text { and } y_{n}=h\left(z^{\prime}, x_{n}\right)\right\}
$$

is called a topological hypersurface in $\mathbb{C}^{n}$.

Let $X$ be a complex manifold of dimension $n$. A subset $A \subset X$ is said to be a topological hypersurface if, for every point $a \in A$, there is a local chart ( $U, \phi$ : $\left.U \rightarrow \mathbb{C}^{n}\right)$ around $a$ such that $\phi(A \cap U)$ is a topological hypersurface in $\mathbb{C}^{n}$

Now let $D \subset X$ be an open subset and let $A \subset \partial D$ be an open subset (with respect to the topology induced on $\partial D$ ). Suppose in addition that $A$ is a topological hypersurface. A point $a \in A$ is said to be of type 1 (with respect to $D$ ) if, for every neighborhood $U$ of $a$ there is an open neighborhood $V$ of $a$ such that $V \subset U$ and $V \cap D$ is a domain. Otherwise, $a$ is said to be of type 2 . We see easily that if $a$ is of type 2, then for every neighborhood $U$ of $a$, there are an open neighborhood $V$ of $a$ and two domains $V_{1}, V_{2}$ such that $V \subset U, V \cap D=V_{1} \cup V_{2}$ and all points in $A \cap V$ are of type 1 with respect to $V_{1}$ and $V_{2}$.

In virtue of Proposition 3.7 in [36] we have the following

Proposition 10.1. Let $X$ be a complex manifold and $D$ an open subset of $X . D$ is equipped with the canonical system of approach regions. Suppose that $A \subset \partial D$ is an open boundary subset which is also a topological hypersurface. Then $A$ is locally pluriregular and $A \subset \widetilde{A}$.

This, combined with Theorem A, implies the following result.

Theorem 10.2. Let $X, Y$ be two complex manifolds, and $D \subset X, G \subset Y$ two nonempty open sets. $D$ (respectively $G$ ) is equipped with the canonical system of approach regions. Let $A$ (respectively $B$ ) be a nonempty open subset of $\partial D$ (respectively $\partial G$ ) which is also a topological hypersurface. Let $Z$ be a complex analytic space possessing the Hartogs extension property. Define

$$
\begin{aligned}
& W:=\mathbb{X}(A, B ; D, G), \\
& \widehat{W}:=\{(z, w) \in D \times G: \omega(z, A, D)+\omega(w, B, G)<1\} .
\end{aligned}
$$

Let $f: W \longrightarrow Z$ be such that:

(i) $f \in \mathcal{C}_{s}(W, Z) \cap \mathcal{O}_{s}\left(W^{o}, Z\right)$;

(ii) $f$ is locally bounded on $W$;

(iii) $\left.f\right|_{A \times B}$ is continuous.

Then there exists a unique mapping $\hat{f} \in \mathcal{O}(\widehat{W}, Z)$ such that

$$
\lim _{\widehat{W} \ni(z, w) \rightarrow(\zeta, \eta)} \hat{f}(z, w)=f(\zeta, \eta), \quad(\zeta, \eta) \in W .
$$


If, moreover, $Z=\mathbb{C}$ and $|f|_{W}<\infty$, then

$$
|\hat{f}(z, w)| \leq|f|_{A \times B}^{1-\omega(z, w)}|f|_{W}^{\omega(z, w)}, \quad(z, w) \in \widehat{W} .
$$

The special case where $Z=\mathbb{C}$ has been proved in [36].

\subsection{System of angular approach regions}

We will use the terminology and the notation in Paragraph 3 of Subsection 2.2. More precisely, if $D$ is an open set of a Riemann surface such that $D$ is good on a nonempty part of $\partial D$, we equip $D$ with the system of angular approach regions supported on this part. Moreover, the notions such as set of positive length, set of zero length, locally pluriregular point which exist on $\partial E$ can be transferred to $\partial D$ using conformal mappings in a local way (see [35] for more details).

Theorem 10.3. Let $X, Y$ be Riemann surfaces and $D \subset X, G \subset Y$ open subsets and $A$ (respectively $B)$ a subset of $\partial D$ (respectively $\partial G$ ) such that $D$ (respectively $G)$ is good on $A$ (respectively $B$ ) and that both $A$ and $B$ are of positive length. Let $Z$ be a complex analytic space possessing the Hartogs extension property. Define

$$
\begin{aligned}
W & :=\mathbb{X}(A, B ; D, G), \quad W^{\prime}:=\mathbb{X}\left(A^{\prime}, B^{\prime} ; D, G\right), \\
\widehat{W} & :=\{(z, w) \in D \times G: \omega(z, A, D)+\omega(w, B, G)<1\}, \\
\widehat{W^{\prime}} & :=\left\{(z, w) \in D \times G: \omega\left(z, A^{\prime}, D\right)+\omega\left(w, B^{\prime}, G\right)<1\right\},
\end{aligned}
$$

where $A^{\prime}$ (respectively $\left.B^{\prime}\right)$ is the set of points at which $A$ (respectively $\left.B\right)$ is locally pluriregular with respect to the system of angular approach regions supported on $A$ (respectively $B)$, and $\omega(\cdot, A, D), \omega\left(\cdot, A^{\prime}, D\right)$ (respectively $\omega(\cdot, B, G)$, $\left.\omega\left(\cdot, B^{\prime}, G\right)\right)$ are calculated using the canonical system of approach regions.

Then for every mapping $f: W \longrightarrow Z$ which satisfies the following conditions:

(i) $f \in \mathcal{C}_{s}(W, Z) \cap \mathcal{O}_{s}\left(W^{o}, Z\right)$;

(ii) $f$ is locally bounded;

(iii) $\left.f\right|_{A \times B}$ is continuous,

there exists a unique mapping $\hat{f} \in \mathcal{O}\left(\widehat{W^{\prime}}, Z\right)$ which admits the angular limit $f$ at all points of $W \cap W^{\prime}$.

If $A$ and $B$ are Borel sets or if $X=Y=\mathbb{C}$ then $\widehat{W}=\widehat{W^{\prime}}$.

If $Z=\mathbb{C}$ and $|f|_{W}<\infty$, then

$$
|\hat{f}(z, w)| \leq|f|_{A \times B}^{1-\omega\left(z, A^{\prime}, D\right)-\omega\left(w, B^{\prime}, G\right)}|f|_{W}^{\omega\left(z, A^{\prime}, D\right)+\omega\left(w, B^{\prime}, G\right)}, \quad(z, w) \in \widehat{W^{\prime}} .
$$

Theorem 10.3 generalizes, in some sense, the result of [35].

In the above theorem we have used the equality $\widehat{\widetilde{W}}=\widehat{W^{\prime}}$ when either $A$ and $B$ are Borel sets or $X=Y=\mathbb{C}$. This follows from the identity $\omega(\cdot, A, D)=$ 
$\widetilde{\omega}(\cdot, A, D)$ when either $A$ is a Borel set or $D \subset \mathbb{C}$ (see [35, Theorem 4.6]). On the other hand, we can sharpen Theorem 10.3 further, namely, hypothesis (i) can be replaced by a weaker hypothesis (i') as follows:

(i') for any $a \in A$ the mapping $\left.f(a, \cdot)\right|_{G}$ is holomorphic and has angular limit $f(a, b)$ at all points $b \in B$, and for any $b \in B$ the mapping $\left.f(\cdot, b)\right|_{D}$ is holomorphic and has angular limit $f(a, b)$ at all points $a \in A$.

To see this it suffices to observe that the hypotheses of Theorem 3.8 and Theorem 6.1 can be weakened considerably when the bounded open set $D$ therein is just one-dimensional.

\subsection{System of conical approach regions}

The remaining part of this section is devoted to two important applications of Theorem A: a boundary cross theorem and a mixed cross theorem. In order to formulate them, we need to introduce some terminology and notation.

Let $X$ be an arbitrary complex manifold and $D \subset X$ an open subset. We say that a set $A \subset \partial D$ is locally contained in a generating manifold if there exist an (at most countable) index set $J \neq \varnothing$, a family of open subsets $\left(U_{j}\right)_{j \in J}$ of $X$ and a family of generating manifolds ${ }^{13}\left(\mathcal{M}_{j}\right)_{j \in J}$ such that $A \cap U_{j} \subset \mathcal{M}_{j}, j \in J$, and that $A \subset \bigcup_{j \in J} U_{j}$. The dimensions of $\mathcal{M}_{j}$ may vary according to $j \in J$. Given a set $A \subset \partial D$ which is locally contained in a generating manifold, we say that $A$ is of positive size if under the above notation $\sum_{j \in J} \operatorname{mes}_{\mathcal{M}_{j}}\left(A \cap U_{j}\right)>0$, where $\operatorname{mes}_{\mathcal{M}_{j}}$ denotes the Lebesgue measure on $\mathcal{M}_{j}$. A point $a \in A$ is said to be a density point of $A$ if it is a density point of $A \cap U_{j}$ on $\mathcal{M}_{j}$ for some $j \in J$. Denote by $A^{\prime}$ the set of density points of $A$.

Suppose now that $A \subset \partial D$ is of positive size. We equip $D$ with the system of conical approach regions supported on $A$. Using the work of B. Jöricke (see, for example, Theorem 3, pages 44-45 in [15]), one can show that ${ }^{14} A$ is locally pluriregular at all density points of $A$. Observe that $\operatorname{mes}_{\mathcal{M}_{j}}\left(\left(A \backslash A^{\prime}\right) \cap U_{j}\right)=$ 0 for $j \in J$. Therefore, it is not difficult to show that $A^{\prime}$ is locally pluriregular. Choose an increasing sequence $\left(A_{n}\right)_{n=1}^{\infty}$ of subsets of $A$ such that $A_{n} \cap U_{j}$ is closed and $\operatorname{mes}_{\mathcal{M}_{j}}\left(\left(A \backslash \bigcup_{n=1}^{\infty} A_{n}\right) \cap U_{j}\right)=0$ for $j \in J$. Observe that $A_{n}^{\prime}$ is locally pluriregular, $\overline{A_{n}^{\prime} \cap U_{j}} \subset A$ for $j \in J$ and that $\widehat{A}:=\bigcup_{n=1}^{\infty} A_{n}^{\prime}$ is locally pluriregular and that $\widehat{A}$ is locally pluriregular at all points of $A^{\prime}$. Consequently, it follows from Definition 2.3 that

$$
\widetilde{\omega}(z, A, D) \leq \omega\left(z, A^{\prime}, D\right), \quad z \in D .
$$

13 A differentiable submanifold $\mathcal{M}$ of a complex manifold $X$ is said to be a generating manifold if for all $\zeta \in \mathcal{M}$, every complex vector subspace of $T_{\zeta} X$ containing $T_{\zeta} \mathcal{M}$ coincides with $T_{\zeta} X$.

14 A complete proof will be available in [29]. 
This estimate, combined with Theorem A, implies the following result which is a generalization in higher dimensions of Theorem 10.3.

Theorem 10.4. Let $X, Y$ be two complex manifolds, let $D \subset X, G \subset Y$ be two open sets, and let $A$ (respectively $B$ ) be a subset of $\partial D$ (respectively $\partial G$ ). $D$ (respectively $G)$ is equipped with a system of conical approach regions $\left(\mathcal{A}_{\alpha}(\zeta)\right)_{\zeta \in \bar{D}, \alpha \in I_{\zeta}}$ (respectively $\left(\mathcal{A}_{\beta}(\eta)\right)_{\eta \in \bar{G}, \beta \in I_{\eta}}$ ) supported on $A$ (respectively on $B$ ). Suppose in addition that $A$ and $B$ are of positive size. Let $Z$ be a complex analytic space possessing the Hartogs extension property. Define

$$
\begin{aligned}
& W^{\prime}:=\mathbb{X}\left(A^{\prime}, B^{\prime} ; D, G\right), \\
& \widehat{W^{\prime}}:=\left\{(z, w) \in D \times G: \omega\left(z, A^{\prime}, D\right)+\omega\left(w, B^{\prime}, G\right)<1\right\},
\end{aligned}
$$

where $A^{\prime}$ (respectively $\left.B^{\prime}\right)$ is the set of density points of $A$ (respectively $B$ ).

Then, for every mapping $f: W \longrightarrow Z$ which satisfies the following conditions:

- $f \in \mathcal{C}_{s}(W, Z) \cap \mathcal{O}_{s}\left(W^{o}, Z\right)$;

- $f$ is locally bounded;

- $\left.f\right|_{A \times B}$ is continuous,

there exists a unique mapping $\hat{f} \in \mathcal{O}\left(\widehat{W^{\prime}}, Z\right)$ which admits $\mathcal{A}$-limit $f(\zeta, \eta)$ at every point $(\zeta, \eta) \in W \cap W^{\prime}$.

If, moreover, $Z=\mathbb{C}$ and $|f|_{W}<\infty$, then

$$
|\hat{f}(z, w)| \leq|f|_{A \times B}^{1-\omega\left(z, A^{\prime}, D\right)-\omega\left(w, B^{\prime}, G\right)}|f|_{W}^{\omega\left(z, A^{\prime}, D\right)+\omega\left(w, B^{\prime}, G\right)}, \quad(z, w) \in \widehat{W^{\prime}} .
$$

The second application is a very general mixed cross theorem.

Theorem 10.5. Let $X, Y$ be two complex manifolds, let $D \subset X, G \subset Y$ be open sets, let $A$ be a subset of $\partial D$, and let $B$ be a subset of $G . D$ is equipped with the system of conical approach regions $\left(\mathcal{A}_{\alpha}(\zeta)\right)_{\zeta \in \bar{D}, \alpha \in I_{\zeta}}$ supported on $A$ and $G$ is equipped with the canonical system of approach regions $\left(\mathcal{A}_{\beta}(\eta)\right)_{\eta \in \bar{G}}, \beta \in I_{\eta}$. Suppose in addition that $A$ is of positive size. Let $Z$ be a complex analytic space possessing the Hartogs extension property. Define

$$
\begin{aligned}
& W^{\prime}:=\mathbb{X}\left(A^{\prime}, B^{*} ; D, G\right), \\
& \widehat{W^{\prime}}:=\left\{(z, w) \in D \times G: \omega\left(z, A^{\prime}, D\right)+\omega\left(w, B^{*}, G\right)<1\right\},
\end{aligned}
$$

where $A^{\prime}$ is the set of density points of $A$ and $B^{*}$ denotes, as usual (see Subsection 2.1 above), the set of points in $\bar{B} \cap G$ at which $B$ is locally pluriregular.

Then, for every mapping $f: W \longrightarrow Z$ which satisfies the following conditions:

- $f \in \mathcal{C}_{s}(W, Z) \cap \mathcal{O}_{s}\left(W^{o}, Z\right)$;

- $f$ is locally bounded along $A \times G$, 
there exists a unique mapping $\hat{f} \in \mathcal{O}\left(\widehat{W^{\prime}}, Z\right)$ which admits $\mathcal{A}$-limit $f(\zeta, \eta)$ at every point $(\zeta, \eta) \in W \cap W^{\prime}$.

If, moreover, $Z=\mathbb{C}$ and $|f|_{W}<\infty$, then

$$
|\hat{f}(z, w)| \leq|f|_{A \times B}^{1-\omega\left(z, A^{\prime}, D\right)-\omega\left(w, B^{*}, G\right)}|f|_{W}^{\omega\left(z, A^{\prime}, D\right)+\omega\left(w, B^{*}, G\right)}, \quad(z, w) \in \widehat{W^{\prime}} .
$$

Concluding remarks. In ongoing joint-works with Pflug [31,32] we develop new cross theorems with singularities. On the other hand, in [37] the problem of optimality of the envelope of holomorphy $\widehat{\widetilde{W}}$ in Theorem A has been investigated. A survey on recent developments in the theory of separately holomorphic mappings could be found in [30].

\section{References}

[1] R. A. Airapetyan and G. M. Henkin, Analytic continuation of CR-functions across the "edge of the wedge", Dokl. Akad. Nauk SSSR 259 (1981), 777-781 (Russian). English transl.: Soviet Math. Dokl. 24 (1981), 128-132.

[2] R. A. AIRAPETYAN and G. M. HENKIN, Integral representations of differential forms on Cauchy-Riemann manifolds and the theory of CR-functions. II, Mat. Sb. 127 (1985), 92112, (Russian). English transl.: Math. USSR-Sb. 55 (1986), 99-111.

[3] O. Alehyane and J. M. HeCART, Propriété de stabilité de la fonction extrémale relative, Potential Anal. 21 (2004), 363-373.

[4] K. AdACHI, M. SUZUKI and M. Yoshida, Continuation of holomorphic mappings with values in a complex Lie group, Pacific J. Math. 47 (1973), 1-4.

[5] O. AlehyANE and A. ZeriAhI, Une nouvelle version du théorème d'extension de Hartogs pour les applications séparément holomorphes entre espaces analytiques, Ann. Polon. Math. 76 (2001), 245-278.

[6] E. BEDFORD, "The operator $\left(d d^{c}\right)^{n}$ on Complex Spaces", Semin. P. Lelong - H. Skoda, Analyse, Années 1980/81, Lect. Notes Math. 919 (1982), 294-323.

[7] E. BEDFORD and B. A. TAYLOR, A new capacity for plurisubharmonic functions, Acta Math. 149 (1982), 1-40.

[8] S. Bernstein, "Sur l'Ordre de la Meilleure Approximation des Fonctions Continues par des Polynômes de Degré Donné", Bruxelles, 1912.

[9] L. M. DRUŻKowsKI, A generalization of the Malgrange-Zerner theorem, Ann. Polon. Math. 38 (1980), 181-186.

[10] A. Edigarian, Analytic discs method in complex analysis, Diss. Math. 402 (2002), 56.

[11] G. M. Goluzin, " Geometric Theory of Functions of a Complex Variable", (English), Providence, R. I.: American Mathematical Society (AMS). VI, 1969, p. 676.

[12] A. A. Gonchar, On analytic continuation from the "edge of the wedge" theorem, Ann. Acad. Sci. Fenn. Math. Diss. 10 (1985), 221-225.

[13] A. A. Gonchar, On Bogolyubov's "edge-of-the-wedge" theorem, Proc. Steklov Inst. Math. 228 (2000), 18-24.

[14] F. HARTOGS, Zur Theorie der analytischen Funktionen mehrer unabhängiger Veränderlichen, insbesondere über die Darstellung derselben durch Reihen, welche nach Potenzen einer Veränderlichen fortschreiten, Math. Ann. 62 (1906), 1-88.

[15] B. JÖRICKE, The two-constants theorem for functions of several complex variables, (Russian), Math. Nachr. 107 (1982), 17-52.

[16] B. JosEFSON, On the equivalence between polar and globally polar sets for plurisubharmonic functions on $\mathbb{C}^{n}$, Ark. Mat. 16 (1978), 109-115. 
[17] S. M. IVASHKOVICH, The Hartogs phenomenon for holomorphically convex Kähler manifolds, Math. USSR-Izv. 29 (1997), 225-232.

[18] M. JARnicki and P. Pflug, "Extension of Holomorphic Functions", de Gruyter Expositions in Mathematics n. 34, Walter de Gruyter, 2000.

[19] M. JARNICKI and P. PFLUG, Invariant distances and metrics in complex analysisrevisited, Dissertationes Math. (Rozprawy Math.) 430 (2005).

[20] M. KLIMEK, "Pluripotential Theory", London Mathematical society monographs, Oxford Univ. Press., n. 6, 1991.

[21] H. Komatsu, A local version of Bochner's tube theorem, J. Fac. Sci., Univ. Tokyo, Sect. I A 19 (1972), 201-214.

[22] F. LÁruSSON and R. SigurdSSON, Plurisubharmonic functions and analytic discs on manifolds, J. Reine Angew. Math. 501 (1998), 1-39.

[23] NGuyên Thanh VÂn, Separate analyticity and related subjects, Vietnam J. Math. 25 (1997), 81-90.

[24] NGUYÊN THANH VÂN, Note on doubly orthogonal system of Bergman, Linear Topological Spaces and Complex Analysis 3 (1997), 157-159.

[25] NGUyên Thanh VÂN and A. ZERIAHI, Familles de polynômes presque partout bornées, Bull. Sci. Math. 107 (1983), 81-89.

[26] NGuyên Thanh VÂN and A. Zeriahi, Une extension du théorème de Hartogs sur les fonctions séparément analytiques, In: " Analyse Complexe Multivariable, Récents Développements", A. Meril (ed.), EditEl, Rende, 1991, 183-194.

[27] NGUYÊN Thanh VÂN and A. Zeriahi, Systèmes doublement orthogonaux de fonctions holomorphes et applications, Banach Center Publ. 31 (1995), 281-297.

[28] V.-A. NGUYÊN, A general version of the Hartogs extension theorem for separately holomorphic mappings between complex analytic spaces, Ann. Scuola Norm. Sup. Pisa Cl. Sci. (V) 4 (2005), 219-254.

[29] V.-A. NGUYÊN, Conical plurisubharmonic measure and new cross theorems, in preparation.

[30] V.-A. NGUYÊN, Recent developments in the theory of separately holomorphic mappings, http://publications.ictp.it, IC/2007/074, 28 pages.

[31] V.-A. NGUYÊN and P. PfLug, Boundary cross theorem in dimension 1 with singularities, Indiana Univ. Math. J., to appear.

[32] V.-A. NGUYÊN and P. PfLUG, Cross theorems with singularities. http://publications.ictp.it, IC/2007/073.

[33] P. PFLUG, Extension of separately holomorphic functions-a survey 1899-2001, Ann. Polon. Math. 80 (2003), 21-36.

[34] P. PFLUG and V.-A. NGUYÊN, A boundary cross theorem for separately holomorphic functions, Ann. Polon. Math. 84 (2004), 237-271.

[35] P. Pflug and V.-A. NGUYÊN, Boundary cross theorem in dimension 1, Ann. Polon. Math. 90 (2007), 149-192.

[36] P. Pflug and V.-A. NGUYÊN, Generalization of a theorem of Gonchar, Ark. Mat. 45 (2007), 105-122.

[37] P. Pflug and V.-A. Nguyên, Envelope of holomorphy for boundary cross sets, Arch. Math. (Basel) 89 (2007), 326-338.

[38] E. A. Poletsky, Plurisubharmonic functions as solutions of variational problems, In: "Several Complex Variables and Complex Geometry", Proc. Summer Res. Inst., Santa Cruz/CA (USA) 1989, Proc. Symp. Pure Math. Vol. 52, Part 1, 1991, 163-171.

[39] E. A. Poletsky, Holomorphic currents, Indiana Univ. Math. J. 42 (1993), 85-144.

[40] T. RAnsford, "Potential Theory in the Complex Plane", London Mathematical Society Student Texts, n. 28, Cambridge: Univ. Press., 1995.

[41] J. P. RosAY, Poletsky theory of disks on holomorphic manifolds, Indiana Univ. Math. J. 52 (2003), 157-169. 
[42] B. ShIFFman, Extension of holomorphic maps into Hermitian manifolds, Math. Ann. 194 (1971), 249-258.

[43] B. ShIfFman, Hartogs theorems for separately holomorphic mappings into complex spaces, C. R. Acad. Sci. Paris Sér. I Math. 310 (1990), 89-94.

[44] J. SICIAK, Analyticity and separate analyticity of functions defined on lower dimensional subsets of $\mathbb{C}^{n}$, Zeszyty Nauk. Univ. Jagiellon. Prace Mat. 13 (1969), 53-70.

[45] J. SiCIAK, Separately analytic functions and envelopes of holomorphy of some lower dimensional subsets of $\mathbb{C}^{n}$, Ann. Polon. Math. 22 (1970), 145-171.

[46] V. P. ZAHARIUTA, Separately analytic functions, generalizations of the Hartogs theorem and envelopes of holomorphy, Math. USSR-Sb. 30 (1976), 51-67.

[47] M. ZERNER, Quelques résultats sur le prolongement analytique des fonctions de variables complexes, Séminaire de Physique Mathématique.

[48] A. ZERIAHI, Comportement asymptotique des systèmes doublement orthogonaux de Bergman: Une approche élémentaire, Vietnam J. Math. 30 (2002), 177-188.

[49] H. WU, Normal families of holomorphic mappings, Acta Math. 119 (1967), 193-233.

Vietnamese Academy of Science and Technology

Institute of Mathematics

Department of Analysis

18 Hoang Quoc Viet Road, Cau Giay District

10307 Hanoi, Vietnam

nvanh@math.ac.vn 